A

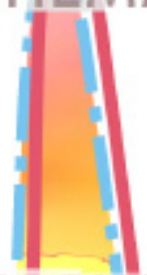

GENERATION

AND

FORWARD PROPAGATION

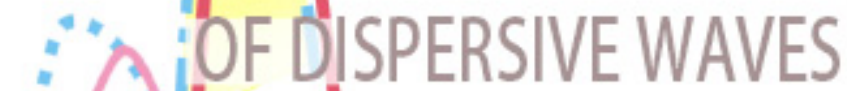

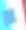

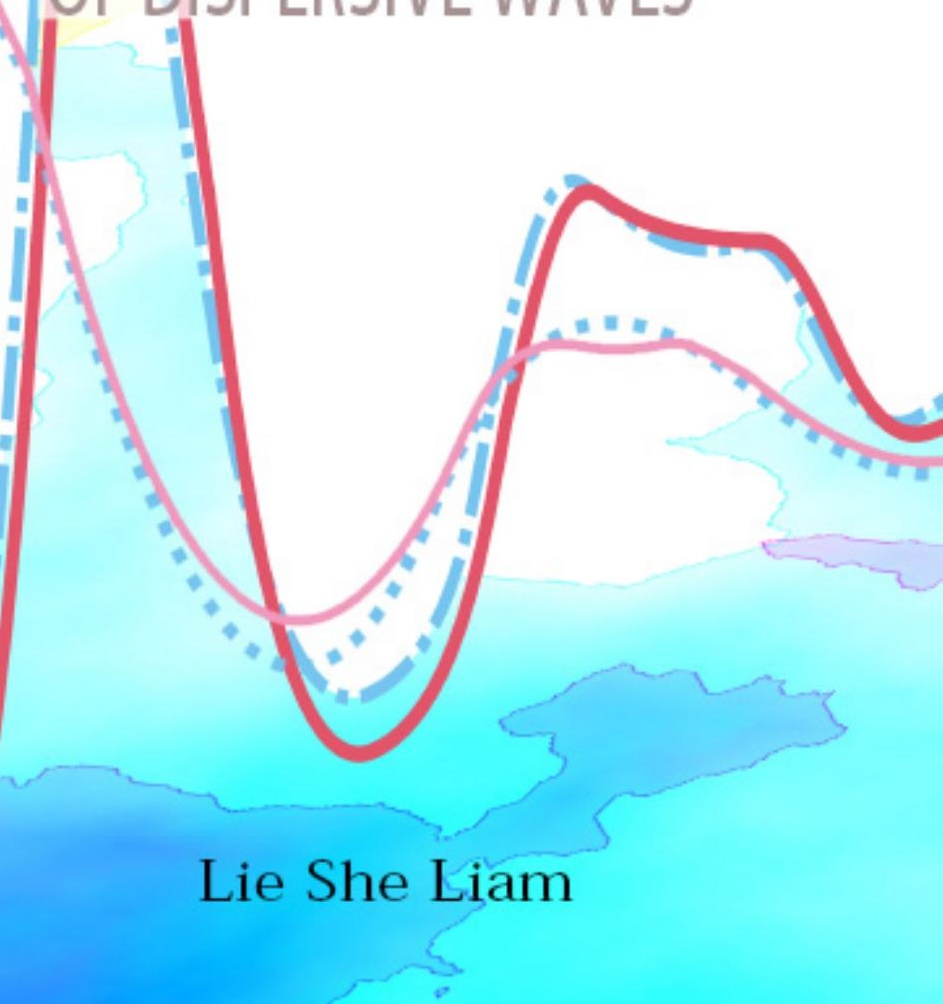




\section{MATHEMATICAL MODELLING OF GENERATION AND \\ FORWARD PROPAGATION OF DISPERSIVE WAVES}

Lie She Liam 


\section{Colophon}

The research presented in this dissertation was carried out at the Applied Analysis and Mathematical Physics (AAMP) group, Department of Applied Mathematics, University of Twente, The Netherlands and Laboratorium Matematika Indonesia (LabMath-Indonesia), Indonesia.

This work has been supported by Netherlands Science Foundation NWO-STW under project TWI-7216.

This thesis was typeset in $\mathrm{IAT}_{\mathrm{E}} \mathrm{X}$ by the author and printed by Gildeprint Printing Service, Enschede, The Netherlands.

(c) Lie She Liam, 2013.

All right reserved. No part of this work may be reproduced, stored in a retrieval system, or transmitted in any form or by any means, electronic, mechanical, photocopying, recording, or otherwise, without prior permission from the copyright owner.

ISBN 978-90-365-3549-6 


\title{
MATHEMATICAL MODELLING OF GENERATION AND FORWARD PROPAGATION OF DISPERSIVE WAVES
}

\author{
DISSERTATION
}

to obtain

the degree of doctor at the University of Twente, on the authority of the rector magnificus,

Prof. dr. Brinksma, on account of the decision of the graduation committee to be publicly defended on Wednesday $15^{\text {th }}$ May 2013 at 12.45

by

\section{Lie She Liam}

born at $19^{\text {th }}$ April 1984

in Jakarta, Indonesia 
This dissertation has been approved by the promotor, prof. dr. ir. E. W. C. van Groesen 
Untuk mereka yang tercinta Papa Lie Tjoen Men \& Mama Sim Fung Tjen 


\section{Acknowledgments}

The work presented here would have never been accomplished without the support and the involvement of many others, to whom I owe an expression of gratitude.

I am grateful to my promotor, Prof. Brenny van Groesen for giving me an opportunity to do a research under his enthusiastic supervision. I would like to thank to Prof. Rene Huijsmans, Prof. Bernard Geurts, Prof. Hoeijmakers and Dr. Gerbrant van Vledder for their willingness to be my committee members. Special thanks to Dr. Tim Bunnik for allowing me to work at MARIN hydrodynamics laboratory during spring period in 2009 and also to Dr. Andonowati for providing me a very nice place to work during my stay in Bandung, Indonesia.

I thank to my teacher Dr. Gerard Jeurnink for involving me as his assistant in several analysis classes. Also thank to Prof. Stephan van Gills who has given me a lot of flexibilities and supports when I worked at his group. I express my sincere thanks to the secretary of AAMP group: Marielle and Linda for their kindness in taking care and arranging everything for my graduation.

It must be very difficult for me to stay in Bandung to finish this book without the support and the presence of my friends: Ruddy Kurnia, Mourice Woran, Andreas Parama, Andy Schauff, Bayu Anggera, Willy Budiman, Fenfen, Wisnu, Mulyadi, Adam, Virginy, Ivanky and Sinatra Kho. Thank you very much for a very warm friendship! My highest appreciation to my friend Meirita Rahmadani who has designed the cover of this thesis. Thank you so much! 
viii

I also would like to thank to my former colleagues for a fruitful discussion about anything: Ivan Lakhturov, Gert Klopman, Natanael, Helena Margareta, Sena, Vita, Ari, Wenny, Arnida, Marcell Lourens, Sid Visser, Shavarsh, Didit, David Lopez, Julia Mikhal, Lilya Ghazaryan and Alyona Ivanova.

My stay in the Netherlands will be different without favors from tante Soefiyatie Hardjosumarto and Ingrid Proost. Thank you very much for a nice chat in the evening and for teaching me how to deal with Dutch culture.

Finally I am grateful to the member of She Lie group especially my dear sisters Lie She Khiun and Lie She Yauw for their unconditional support and encouragement in everything that I do. Terima kasih juga untuk pria dan wanita yang besar hatinya telah melebihi tubuhnya sendiri: papa Lie dan mama Sim, terima kasih untuk semua kebaikan kalian.

Enschede, April 2013

Lie She Liam 


\section{Summary}

This dissertation concerns the mathematical theory of forward propagation and generation of dispersive waves. We derive the AB2-equation which describes forward traveling waves in two horizontal dimension. It is the generalization of the Kadomtsev-Petviashvilli (KP) equation. The derivation is based on the variational principle of water waves. Similar to its predecessor, the $\mathrm{AB}$-equation, the $\mathrm{AB} 2$-equation is dispersive, accurate in second order and can be adjusted for any water depth. Using pseudo-spectral method, the numerical implementation of the AB2-equation can be done easily since exact dispersion is described by a nonpolynomial pseudo-differential operator that can easily be dealt with in spectral space.

For wave generation, we derive various models that describe excitation if the wave elevation (or fluid potential) at a certain position is given. The wave generation discussed in this dissertation is done by an embedded source term added to the equation(s) of water wave motion. In this way, we transform the problem of homogeneous boundary value problem into an inhomogeneous problem. We derive the source functions for any kind of waves to be generated and for any dispersive equation including the general case of (linear) dispersive Boussinesq equations. For a dispersive wave equation, the source is not unique; many choices can be taken as long as they satisfy a certain source - influx signal relation. This is different from the actual condition in a hydrodynamic laboratory where there is a one to one correspondence between influx signal and the generated waves.

We designed a set of experiments for oblique wave interaction. The aim of the experiment is to test the applicability and the performance of 
the AB2-equation and the influxing technique. These experiments were executed in a water tank of MARIN hydrodynamic laboratory. The experiments are performed by generating two oblique waves from two sides of the basin and let the waves collide. We compare the measurements from the experiments and the AB2 simulation results. The AB2 simulations and the MARIN measurements are in satisfactory agreement, showing the bichromatic beat wave pattern, even for large nonlinear effects. 


\section{Contents}

Acknowledgments vii

Summary ix

Contents $\quad x i$

1 Introduction $\quad \mathbf{1}$

1.1 Water wave investigations ............. . 2

1.1.1 Classical era of the development of water wave theory - until $19^{\text {th }}$ century . . . . . . . . . 2

1.1.2 Contemporary era of the development of water wave theory - after $19^{\text {th }}$ century ......... 6

1.2 Present contributions . . . . . . . . . . . . . 10

1.2.1 The AB2-equation ............. . . 11

1.2.2 The Embedded influxing technique . . . . . . . . 12

1.3 Outline of the dissertation . . . . . . . . . . . 13

2 Variational derivation of improved KP-type of equations 17

2.1 Introduction ................... . . . . . 18

2.2 Variational structure . . . . . . . . . . . . . . 20

2.3 Mainly unidirectional linear waves ........... . . 23

2.4 Nonlinear AB2-equation . . . . . . . . . . . . . . . 26

2.5 Approximations of the AB2-equation .......... . 29

2.6 Conclusion and remarks . . . . . . . . . . . . . . 30 
3 Embedded wave generation for dispersive wave models 31

3.1 Introduction . . . . . . . . . . . . . . . . . 31

3.2 Forward propagating dispersive wave models . . . . . . . 33

3.2 .1 Definitions and notation . . . . . . . . . . . 33

3.2 .2 1D uni-directional waves . . . . . . . . . . . . 36

3.2.3 2D forward dispersive wave model . . . . . . . . 42

3.3 Multi-directional propagating dispersive wave models . . . . 45

3.3.1 Second order dispersive wave model . . . . . . . . 46

3.3.2 1D Hamiltonian wave model . . . . . . . . . . . . 47

3.3.3 2D Hamiltonian wave model . . . . . . . . . . . 52

3.4 Numerical simulations . . . . . . . . . . . . . . . 55

3.4.1 1D Spectral implementation: Nonlinear wave focusing 56

3.4.2 Finite element implementation: Uni-directional influxing . . . . . . . . . . . . . . . 59

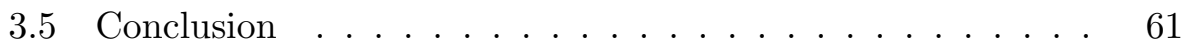

4 Experiment and simulation of oblique wave interaction 63

4.1 Introduction . . . . . . . . . . . . . . . . 63

4.2 Experimental setting . . . . . . . . . . . 66

4.2.1 Physical outlook of water basin . . . . . . . . 66

4.2 .2 Designed test cases . . . . . . . . . . . . . . 67

4.3 Mathematical modeling and simulation . . . . . . . . . 72

4.4 Comparison between MARIN experiment and AB2-simulation 79

4.5 Conclusion . . . . . . . . . . . . . . 9 95

5 Conclusions and recommendations $\quad 97$

5.1 Conclusions . . . . . . . . . . . . . . . . 97

5.2 Recommendations . . . . . . . . . . . . . . . 99

$\begin{array}{ll}\text { Bibliography } & 101\end{array}$ 


\section{Chapter 1}

\section{Introduction}

Waves, as visible on the surface of oceans and seas, cannot exist by themselves for they are mainly caused by winds. The wind transfers its energy to the water surface and makes it move. Part of this energy is contained in waves as they rise and fall, known as potential energy, and another part of the energy is moving together with the waves, known as kinetic energy.

The energy contained in water waves can bring both beneficial and adverse effects for human activities. On the beneficial side, water waves can be used as a source for renewable energy. For instance in Portugal where the world's first commercial wave farm, the Aguçadoura wave farm, is located, several Pelamis Wave Energy Machinery Converter are installed to transform energy from water waves into an enormous amount of electric power [60]. Another wave farm which is planned to be the largest in the world can be found in Scotland, United Kingdom [60].

On the other side, the adverse effects of water waves have been documented in many media. Following are some of the excerpts from those media:

- BBC News - 3 March 2010 : " Two people have been killed and six injured as the 8 meters high giant waves slammed into a cruise ship in the Mediterranean... The rogue waves hit the Cypriot-owned Louis Majesty off the coast of north-east Spain and broke ship windows." 
- "In January 2007, the 18 meters fishing boat Starrigavan, while trying to cross the bar of Tillamook Bay along the Oregon coast, was hit by three 6 meters waves and rolled three times... Crew members was killed and the vessel was thrown onto the jetty. "Source: [27].

- In August 2004, National Geographic published an article about "monster" waves in sea. It said that "During the last two decades, more than 200 supertankers-ships over 200 meters (656 feet) long have sunk beneath the waves. Rogue waves are thought to be the cause for many of these disasters, perhaps by flooding the main hold of these giant container ships... Offshore oil rigs also get hit by rogue waves. Radar reports from the North Sea's Gorm oil field show 466 roguewave encounters in the last 12 years."

These documentations show that waves can be dangerous and harmful for ships, oil platforms and other marine infrastructures. Therefore, understanding of waves is an utmost essential thing for naval engineers and scientists. In the next section of this chapter we will have a closer look at the efforts that have been spent by scientists to investigate water waves.

\subsection{Water wave investigations}

This section will only cover the main inventions scientist made that are closely related to what this dissertation is aiming for.

\subsubsection{Classical era of the development of water wave theory - until $19^{\text {th }}$ century}

The study of water waves started in $17^{\text {th }}$ century. It was Isaac Newton who was the first to attempt to formulate a theory of water waves [7]. In 1687, in Book II, Prop. XLV of Principia, Newton mentioned that the frequency of deep-water waves must be proportional to the inverse of the square root of the breadth of the wave. 
In 1757, Leonhard Euler published his work on equations for inviscid (no viscosity) flow. The equations represent conservation of mass (continuity) and momentum (Newton's second law). The equations are valid for compressible as well as for incompressible flow.

Laplace in 1776 posed a general initial value problem which leads him to (what we know nowadays as) Laplace's equation: Given any localized initial disturbance of the water surface, what is the subsequent motion? Cauchy and Poisson later addressed this problem at great length $[7,14,17]$. At the same time, the influence of wind speed and increase of the water-level at coasts is documented for the first time by Maitz de Goimpy. It is based on his observation that closely agrees with theoretical hypotheses that the wave speed is directly proportional to the wind speed. Lagrange in 1781 derived the linear governing equations for small-amplitude waves, and obtained the solution in the limiting case of long plane waves in shallow water; he found that the propagation speed of waves will be independent of wavelength and proportional to the square root of the water depth.

In December 1813, the French Académie des Sciences announced a mathematical prize competition on propagation of infinitely deep water wave. Cauchy won the prize and his work was published in 1827. Cauchy employed Fourier method to analyze the following Laplace equation

$$
\frac{\partial^{2} \Phi}{\partial x^{2}}+\frac{\partial^{2} \Phi}{\partial y^{2}}+\frac{\partial^{2} \Phi}{\partial z^{2}}=0
$$

together with the linearized free surface condition:

$$
g \frac{\partial \Phi}{\partial z}+\frac{\partial^{2} \Phi}{\partial t^{2}}=0
$$

Here $\Phi(x, y, z)$ denotes the velocity potential at the $x, y$ (horizontal) coordinates and $z$ (vertical) coordinate. Cauchy then took the second time derivative of (1.2) and obtained:

$$
\frac{\partial^{4} \Phi}{\partial t^{4}}=-g \frac{\partial^{3} \Phi}{\partial t^{2} \partial z}=-g \frac{\partial^{3} \Phi}{\partial z \partial t^{2}}=g^{2} \frac{\partial^{2} \Phi}{\partial z^{2}} .
$$


Using equation(1.1) then

$$
\frac{\partial^{4} \Phi}{\partial t^{4}}+g^{2}\left(\frac{\partial^{2} \Phi}{\partial x^{2}}+\frac{\partial^{2} \Phi}{\partial y^{2}}\right)=0
$$

Assuming periodic waves of form $\exp \left[i\left(k_{x} x+k_{y} y-\omega t\right)\right]$, the previous result of Newton is recovered, i.e:

$$
\omega^{2}=g\left(k_{x}^{2}+k_{y}^{2}\right)^{1 / 2} .
$$

However the investigation of Cauchy is only valid for the (linear) deep water case since Cauchy neglected the bottom boundary condition.

Sixty five years after Laplace posted a question about wave motion, Airy in 1841 gave a complete formulation of linear wave theory which include the impermeable boundary-condition into Cauchy's formulation; the equations are as follows $[7,10]$ :

$$
\begin{array}{rlrl}
\frac{\partial^{2} \Phi}{\partial x^{2}}+\frac{\partial^{2} \Phi}{\partial y^{2}}+\frac{\partial^{2} \Phi}{\partial z^{2}} & =0 ; & & \text { for }-h \leq z \leq 0 \\
\frac{\partial \Phi}{\partial t}+g \eta=0 ; & \text { for } z=0 \\
\frac{\partial \eta}{\partial t}=\frac{\partial \Phi}{\partial z} ; & \text { for } z=0 \\
\frac{\partial \Phi}{\partial z}=0 ; & \text { for } z=-h,
\end{array}
$$

with $\eta$ the surface elevation and $h, g$ water depth and gravitational acceleration respectively. Observe that combining the free surface condition (1.5) and (1.6) we obtain the condition (1.2) of Cauchy. Airy's linear theory is accurate for small ratios of the wave height to water depth (for waves in finite depth), and wave height to wavelength (for waves in deep water). As a consequence of Airy's theory, the (linear) dispersion relation for arbitrary water depth was introduced. For a propagating wave of a single frequency, a monochromatic wave, is of the form: $\eta(x, y, t)=a \cos \left(k_{x} x+k_{y} y-\omega t\right)$. Then, in order for $\eta$ to be a solution of equation(1.4)-(1.7), the following (dispersion) relation should be satisfied:

$$
\omega^{2}=g k \tanh (k h),
$$


with $k=\sqrt{k_{x}^{2}+k_{y}^{2}}$ and $h$ the water depth. So, frequency $\omega$ and wavenumber $k$, or equivalently period $\mathrm{T}$ and wavelength $\lambda$, cannot be chosen independently, but are related. The dispersion relation also tells that each wave travels with its own speed (which is the quotient of $\omega$ and $k$ ), with the consequence that shorter waves travel slower.

Six years after Airy's theory was published, Stokes in 1847 published his work on a nonlinear wave theory which is accurate up to third order in wave steepness $[8,14]$. He showed that in deep water there exists a periodic wave for which the profile is no longer sinusoidal. This profile is given as [59]:

$$
\eta(x, t)=a \cos \left(k x-\omega_{s} t\right)+\frac{1}{2} k a^{2} \cos 2\left(k x-\omega_{s} t\right)+\frac{3}{8} k^{2} a^{3} \cos 3\left(k x-\omega_{s} t\right)
$$

with $\omega_{s}^{2}=g k\left(1+k a^{2}\right)$. Stokes introduced the nonlinear effect that leads to the result that the dispersion relation involves the amplitude. As a consequence, the steeper the wave the faster it travels. A typical example of a Stokes wave profile is depicted in figure(1.1). Observe that at a crest Stokes wave is getting higher and it is flatter at a trough.

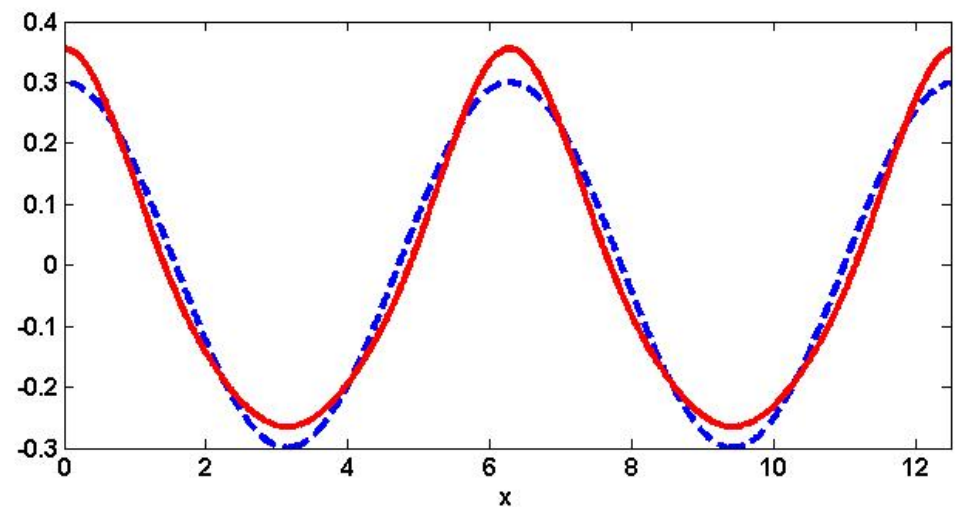

Figure 1.1: Plot of Stokes third order wave profile (solid line) and harmonic wave profile(dashed line) for $k=1, a=0.3$ and $t=0$. 
In 1872, Joseph Boussinesq derived the equations known nowadays as the (original) Boussinesq equation. It assumed waves to be weakly nonlinear and fairly long. It incorporated frequency dispersion (as opposite to the shallow water equations, which are not frequency-dispersive). The idea behind Boussinesq's work is the reduction of spatial dimension by eliminating the vertical coordinate from the equation of motion. This can be done by using Taylor expansion up to a certain order around still water level at the waves velocity potential function.

While the Boussinesq equation allows for waves traveling simultaneously in opposite directions, it is often advantageous to only consider waves traveling in one direction. One earliest of such one directional models is what we know as the KdV-equation. It was named after Diederik Korteweg and Gustav de Vries who derived it in 1895 . For weakly nonlinear and weakly dispersive long waves, the equation in a frame of reference moving with the speed $\sqrt{g h}$ and written in normalized variables, is given as follows:

$$
\eta_{t}+6 \eta \eta_{x}+\eta_{x x x}=0,
$$

with $\eta$ the wave elevation that travels in the positive $x$-direction. See for instance Johnson [18] for the derivation. The KdV-equation shares the same property with the Boussinesq (1872) model that both have (periodic) cnoidal and soliton profiles as solution. The soliton is an interesting solution since it shows the balancing of dispersive and nonlinear effects which has no counterparts in the linear dispersive wave theory. The soliton profile was also the answer for the (theoretical) existence of the experimental result of Scott Russell in 1844.

\subsubsection{Contemporary era of the development of water wave theory - after $19^{\text {th }}$ century}

Many efforts to find better equations that are capable to describe the motion of waves have been made after $19^{\text {th }}$ century (and until the time of writing this dissertation). Most of these efforts are to improve the accuracy of the model in terms of dispersion and nonlinear properties. The expense of doing this is the complication in the model, i.e. by including higher order spatial 
derivatives, some of which are mixed spatial-temporal derivatives. As a consequence, the numerical implementation can become a serious challenge, in particular if one is interested in two dimensional spatial surface wave propagation.

In this section, instead of listing those mathematical model improvements, the development of the so-called variational formulation for water waves is summarized. This formulation is using an optimization approach to a certain functional.

In 1967, Luke formulated a variational description for the gravity driven irrotational motion of a layer of incompressible fluid with a free surface $[29,49]$ as:

$$
\text { crit }_{\eta, \Phi} \int \mathcal{P}(\eta, \Phi) d t
$$

where $\mathcal{P}(\eta, \Phi)=\iint_{-h}^{\eta} \rho\left[\partial_{t} \Phi+\frac{1}{2}\left|\nabla_{3} \Phi\right|^{2}+g z\right] d z \mathbf{d x}$; the fluid density $\rho$ will be taken to be one in the following. The variables in this variational principle are the surface elevation $\eta$ (depending on the two horizontal dimensions $x$ and $y$ ) and the fluid potential $\Phi$ inside the fluid, so depending on horizontal and the vertical dimension. Luke's variational formulation has been noticed before by Bateman in 1929 but without a free surface [4].

Zakharov in 1968 [61] and Broer in 1974 [5] showed the Hamiltonian structure for the dynamics of wave elevation $\eta$ and fluid potential $\phi$, i.e.

$$
\begin{aligned}
& \partial_{t} \eta=\delta_{\phi} \mathcal{H}(\eta, \phi) \\
& \partial_{t} \phi=-\delta_{\eta} \mathcal{H}(\eta, \phi),
\end{aligned}
$$

with $\phi=\Phi(x, y, \eta(x, y))$ the fluid potential at the surface and $\mathcal{H}(\eta, \phi)$ the total energy which is the sum of the potential and kinetic energy:

$$
\mathcal{H}(\eta, \phi)=K(\eta, \phi)+\frac{1}{2} \int g \eta^{2} \mathbf{d x} .
$$

The kinetic energy $K(\eta, \phi)$ is expressed as:

$$
K(\eta, \phi)=\frac{1}{2} \iint_{-h}^{\eta}\left|\nabla_{3} \Phi\right|^{2} d z \mathbf{d x} .
$$


Observe that here the kinetic energy is expressed with the fluid potential $\Phi$ which is defined for all internal fluid instead of $\phi$.

Miles in 1977 [33] showed the relation between equation (1.10)-(1.11) and (1.9). The relation became clear by expressing Luke's Lagrangian as:

$$
\operatorname{Crit}_{\phi, \eta} \int\left\{\int \phi \partial_{t} \eta \mathbf{d} \mathbf{x}-\mathcal{H}(\eta, \phi)\right\} d t .
$$

This is possible by applying Leibniz's rule $\int_{-h}^{\eta} \partial_{t} \Phi d z=\partial_{t}\left(\int_{-h}^{\eta} \Phi d z\right)-\phi \partial_{t} \eta$ to Luke's functional. Due to the work of Miles, Broer and Zakharov, the dimension reduction is achieved; from the vertical and horizontal dependence, the dynamics now is expressed only in the horizontal variables.

The potential energy is easily expressed explicitly as the surface elevation $\eta$. Unfortunately it is not the case with the kinetic energy. The dimension reduction is now depending on the choice of the kinetic energy. Nevertheless several approximations are available for this.

Klopman et al. [22] shows a method of approximating the vertical structure of the fluid potential $\Phi$ in the kinetic energy functional. He applied Ritz technique to decompose $\Phi$ as a limited sum of trial functions in the $z$-direction and functions in the horizontal $\mathbf{x}$-direction, i.e.

$$
\Phi(\mathbf{x}, z, t) \approx \phi(\mathbf{x}, t)+\sum_{m=1}^{M} F_{m}(z) \psi_{m}(\mathbf{x}, t) .
$$

The functions $F_{m}$ are taken a priori and should satisfy $F_{m}(z=\eta)=0$ for all $m$ to reassure the surface condition $\Phi(\mathbf{x}, z, t)=\phi(\mathbf{x}, t)$. There are two types of profiles which are used in the literature for $F_{m}$, namely a single (mode) parabolic profile:

$$
F_{m}^{p}(z)=\frac{1}{2} \frac{(z+h)^{2}-(\eta+h)^{2}}{h+\eta}
$$

and a hyperbolic Airy profile:

$$
F_{m}^{a}(z)=\frac{\cosh \left(k_{m}(z+h)\right)}{\cosh \left(k_{m}(\eta+h)\right)}-1
$$


Substituting this approximation into (1.10) and (1.11) leads to the Variational Boussinesq Model (VBM). See [21, 22] for the model and [2, 3] for a practical application.

A recent investigation on VBM is performed by Lakhturov et al. [23] in 2010. Its aim is to find an optimal choice for the wavenumber(s) $k_{m}$ in the airy profile $F_{m}^{a}(z)$ of VBM. For a single harmonic wave, it is intuitively clear that the choice for such $k_{m}$ will be the related wavenumber in the wave spectra. Yet for an irregular wave for which the spectrum is broad it is not clear which wavenumber(s) needs to be chosen. The idea behind their work is the minimization error between the VBM kinetic energy and the exact kinetic energy. Even though the Optimized VBM is case-dependent, due to its flexibility of choosing (several) vertical profiles $F_{m}^{a}$, the Optimized-VBM will apparently outperform other Boussinesq type models like Peregrine [9], Madsen and Sørensen [30] and Nwogu [34].

In 2007, Van Groesen \& Andonowati in [46] approximated the kinetic energy functional for one horizontal spatial dimension. The kinetic functional is approximated around the still water level and is given by:

$$
\begin{aligned}
K(\eta, \phi) & =\frac{1}{2} \iint_{-h}^{\eta}\left[\left(\partial_{x} \Phi\right)^{2}+\left(\partial_{z} \Phi\right)^{2}\right] d z d x \\
& \approx \frac{1}{2} \int \phi_{0} W_{0}+\eta\left\{\left(\partial_{x} \phi_{0}\right)^{2}+W_{0}^{2}\right\} d x,
\end{aligned}
$$

with $\phi_{0}=\Phi(x, z=0, t)$ and $W_{0}=-\frac{1}{g} C^{2} \partial_{x}^{2} \phi_{0}$ and $\mathrm{C}$ is the phase velocity operator whose symbol is given in Fourier space by: $\hat{C}(k)=\frac{\Omega(k)}{k}$ with $\Omega(k)=\operatorname{sign}(k) \sqrt{g k \tanh (k h)}$ and $h$ the water depth. Neglecting the cubic term of the kinetic energy and substituting this into (1.10)-(1.11) leads to:

$$
\begin{aligned}
\partial_{t} \eta & =-\left(C^{2} / g\right) \partial_{x}^{2} \phi_{0} \\
\partial_{t} \phi_{0} & =-g \eta
\end{aligned}
$$

which can be rewritten as a linear second order dispersive equation:

$$
\partial_{t}^{2} \eta=\partial_{x}^{2} C^{2} \eta
$$


In the same paper, a unidirectional restriction is made for the fluid above flat bottom i.e. $\phi_{0}=g \partial_{x}^{-1} C^{-1} \eta$. With this restriction, the result is the so called AB-equation which describes waves that travel in one direction. Different from the classical KdV-equation in 1895 and the other improvements of it like in $[1,31,45]$ where the $\mathrm{KdV}$ is improved until seventh order yet lacking accuracy in the dispersive term, the AB-equation is valid for any water depth, is exact in dispersion and the nonlinearity is accurate up to and including second order. Keeping the nonlinearity up to second order makes the $\mathrm{AB}$-equation more convenient in numerical implementation and makes the computing time faster while it does not lack in accuracy for most of the practical cases as presented in Van Groesen et al. [50] for the case of narrow spectra of bichromatic waves and Latifah \& Van Groesen [24] for the case of a very broad spectra of focussing wave group and New Year waves. In [47] the model equations of the type of the AB-equation for waves above varying bottom are explained and in [48] some test cases to validate the $\mathrm{AB}$-equation above varying bottom are presented.

\subsection{Present contributions}

There are two main motivations behind writing this dissertation and they serve as (at least) two added-values to the present knowledge of water waves. It is the desire of the writer to derive a new two dimensional wave equation, without a lot of complication in the final formula, which will have exact dispersion and accurate in the nonlinearity and is able to describe forward propagating waves for all water depth. This type of equation will be useful for instance for simulating multi-directional waves in hydrodynamic laboratories.

When engineers at a hydrodynamic laboratory want to perform wave simulations for wave prediction or for maritime infrastructures testing, these simulations are usually done by prescribing a wave elevation signal (as a function of time) at certain positions which are related to the positions of flaps in laboratory. The mathematical model should include this as a boundary condition. Instead of solving this type of boundary value 
problem, an alternative approach is available in the literature such as the work of G. Wei and J.T. Kirby [54], G. Wei, et al. [55] and Kim, et al. [20]. Using various types of Boussinesq equation, they offer an influxing method by adding a source in the governing equation. However there is no systematic and simple approach in the literature; it seems as if there is no general mathematical formula and similarity between one (Boussinesq) equation and the others. This triggered the writer to find an influxing model in an accurate systematic way that is applicable for general setting.

The following subsections give an overview of what has been achieved related to these motivations.

\subsubsection{The AB2-equation}

Research on the AB2-equation is initialized after the writer found the fact that the AB-equation improves significantly the classical KdV-equation. In [50], it is shown that the AB-equation can approximate the Highest Stokes Wave (HSW) that is a Stokes wave in deep water with a profile that has a corner of 120 degrees in the crest. None of the KdV type of equations are able to do this.

When looking into the paper of Kadomtsev \& Petviashvili [19] about the generalization of the KdV-equation in two space dimension, their assumption is similar to the $\mathrm{KdV}$-equation that it is weakly dispersive. The $\mathrm{KP}$-equation is valid for waves that mainly propagate in the $x$-direction; the $y$-coordinate dependence is weak. This brought up the idea to derive the two dimensional version of the $\mathrm{AB}$-equation which is the $\mathrm{AB} 2$-equation. It follows from the same principle as in the AB-equation; dimension reduction for the vertical (z-)coordinate in the kinetic energy functional and the choice of restriction to forward propagating waves. Explicitly, The AB2-equation is given as follows:

$$
\partial_{t} \eta=-\sqrt{g} A_{2}\left[\begin{array}{c}
\eta-\frac{1}{4}\left(A_{2} \eta\right)^{2}+\frac{1}{2} A_{2}\left(\eta A_{2} \eta\right) \\
+\frac{1}{4}\left(B_{2} \eta\right)^{2}+\frac{1}{2} B_{2}\left(\eta B_{2} \eta\right) \\
+\frac{1}{4}\left(\gamma_{2} \eta\right)^{2}+\frac{1}{2} \gamma_{2}\left(\eta \gamma_{2} \eta\right)
\end{array}\right],
$$


with

$$
A_{2}=\frac{i \Omega_{2}}{\sqrt{g}}, B_{2}=\partial_{x} A_{2}^{-1} \text { and } \gamma_{2}=\partial_{y} A_{2}^{-1}
$$

and $\Omega_{2}$ is the operator for two dimensional dispersion relation, its symbol is given in Fourier space as $\Omega_{2}\left(k_{x}, k_{y}\right)=\operatorname{sign}\left(k_{x}\right) \sqrt{g k \tanh (k h)}$ with $k=$ $\sqrt{k_{x}^{2}+k_{y}^{2}}$. Expanding the linear term of the AB2-equation up to second order around $k_{y}=0$ will lead to the improved linear KP-equation, see chapter 2 section 3 for the details. Neglecting the dependency on the $y$ coordinate will give the original (one dimensional) AB-equation.

Since the AB2-equation has similar properties as the AB-equation, it is expected that the AB2-equation performs better than any KP-type of equation.

\subsubsection{The Embedded influxing technique}

The details of the technique described in this section can be found in chapter 3 , here only the one-dimensional case is illustrated.

For any dispersive wave equation it is possible to model wave influxing by adding an embedded source function to the equation of wave motion. The source function(s) is only depending on the dispersive property of the model. As will be shown in chapter 3, for one dimensional case, if the influx signal is $s(t)$ at $x=0$, the embedded source function $S(x, t)$ will be of the following form in Fourier space:

$$
\bar{S}(K(\omega), \omega)=\frac{1}{2 \pi} V_{g}(K(\omega)) \check{s}(\omega),
$$

where $V_{g}=\frac{d \Omega}{d k}$ is the group speed which brings the dispersive information to the influxing model and $K(\omega)$ is the inverse function of the involved dispersion relation. Observe that the source function is uniquely determined in Fourier space for $k$ and $\omega$ satisfying the dispersion relation of the model. However in physical space the source function is not unique. For instance if the source function $S(x, t)$ is a multiplication between a temporal function $f(t)$ and a spatial function $g(x)$, i.e. $S(x, t)=f(t) \cdot g(x)$, then for a given 
$g(x)$ the temporal function $f(t)$ is obtained from the following relation:

$$
\check{f}(\omega)=\frac{1}{2 \pi} \frac{V_{g}(K(\omega)) \check{s}(\omega)}{\hat{g}(K(\omega))} .
$$

From this formula, it is noticeable that waves can be generated by using a different influx signal $f(t)$ instead of the originally prescribed signal $s(t)$. However to have a correct influx, the "modified" signal $f(t)$ should satisfy equation (1.17) and it depends on the modification done in the spatial coordinates. This is different from the actual condition in the hydrodynamic laboratory where there is only a one to one correspondence between influx signal and the generated waves.

In the KdV type of equation the result of the embedded influxing model will be waves propagating in one direction while in the Boussinesq type of equation the result will depend on the choice of spatial function $g(x)$. For a (skew-)symmetric $g(x)$ the result of influxing will be waves propagating in both forward and backward direction (a)symmetrically. By Combining the symmetric and skew-symmetric influxing it is possible to obtain wave propagating in only one direction for a Boussinesq model.

The influxing technique which is described in this dissertation is applied by Van Groesen et al. in [50], Van Groesen \& I.v.d. Kroon in [48], Latifah \& Van Groesen in [24] for the case of one dimensional unidirectional equation and is also applied by Adytia in $[2,3]$ for the variational Boussinesq model. In chapter 4 the influxing method for two dimensional forward propagating waves model is also applied where the result of the model is presented together with the measurement result from the hydrodynamic laboratory.

The derivation of the embedded influxing given in this dissertation is based on the first order linear theory and there is no second order steering theory, as in $[38,39]$, applied here.

\subsection{Outline of the dissertation}

This section describes the main contents of the dissertation and serves as a quick reference to the appropriate chapter. This dissertation consists of 
five chapters for which the order is made as follows. Chapter 2 describes a mathematical model of forward propagating dispersive waves in two dimensional space. Chapter 3 is the result of the investigation on water wave influxing model. In chapter 4 the numerical simulation and experimental results at MARIN are presented and the final chapter contains concluding remarks and suggestions for further research. A brief summary of chapter 2 - chapter 4 of this dissertation is given as follows:

\section{Chapter 2 - Governing equation}

The aim of this chapter is to derive the AB2-equation which is a dispersive and nonlinear wave equation that describes the dynamics of waves in two-dimensions. The derivation of the AB2-equation stems from the variational structure of water waves which is treated in section 2.2. The linear and the nonlinear version of the AB2-equation are presented in section 2.3 and 2.4 respectively. Several approximations which can be made from the AB2 are discussed in section 2.5. The chapter ends with commenting the difference between the AB2-equation and KP-type of equations. This chapter has been published as [42].

\section{Chapter 3 - Embedded wave generation model}

The result of this chapter is a mathematical model which gives a way to influx waves by incorporating a source function into the equation(s) of wave motion. Not only the equation described in chapter 2 but also all Boussinesq type of equations are treated. Section 3.2 deals with embedded wave generation for forward dispersive propagating waves both in one dimension (section 3.2.1) and in two dimensions (section 3.2.2).

For wave influxing in more directions, Boussinesq type of equations in Hamiltonian form are used as the governing equation. Embedded source functions for this type of equation are given in section 3.3, with 3.3.2 for the one dimensional case and 3.3.3 for the two dimensional case. Section 3.3.1 describes influxing by using second order dispersive wave equation. This chapter is closed with some numerical simulations to illustrate the embedded wave generation.

Part of this chapter (until section 3.2.2) is similar to a paper that has been submitted for publication as [41]. 


\section{Chapter 4 - Experiments and simulation}

This chapter deals with experiments on oblique wave interaction that have been conducted at MARIN hydrodynamic laboratory in Wageningen, the Netherlands. The experiments are designed by influxing harmonic waves from two sides of the water tank so that in the middle of the tank the generated waves are expected to be in the form of bichromatic waves. The detail of the experimental setup and the measurement results are given in section 4.2 .

For the numerical simulation, the AB2-equation of chapter 2 is chosen as the governing equation with an appropriate embedded source term derived in chapter 3. Section 4.3 describes the model and the numerical method used for the simulation. The results of the simulation are compared with the data from the laboratory and are presented in section 4.4. 


\title{
Chapter 2
}

\section{Variational derivation of improved KP-type of equations ${ }^{1}$}

\begin{abstract}
The Kadomtsev-Petviashvili equation describes nonlinear dispersive waves which travel mainly in one direction, generalizing the Korteweg - de Vries equation for purely uni-directional waves. In this paper we derive an improved KP-equation that has exact dispersion in the main propagation direction and that is accurate in second order of the wave height. Moreover, different from the KP-equation, this new equation is also valid for waves on deep water. These properties are inherited from the AB-equation [46] which is the unidirectional improvement of the $\mathrm{KdV}$ equation. The derivation of the equation uses the variational formulation of surface water waves, and inherits the basic Hamiltonian structure.
\end{abstract}

\footnotetext{
${ }^{1}$ Published as :

Lie S.L and E. van Groesen. Variational derivation of improved KP-type of equations. Phys.Lett.A, 374: 411-415, 2010.
} 


\subsection{Introduction}

The Kadomtsev-Petviashvilli equation, or briefly the KP-equation, is derived in 1970 as a generalization of the Korteweg- de Vries (KdV)-equation for two spatial dimensions $[18,19,28]$. It is a well-known model for dispersive, weakly nonlinear and almost unidirectional waves. Although the equation is of relevance in many applications with various dispersion relations, we will concentrate in this paper on the application to surface water waves on a - possibly infinitely deep - layer of fluid; for other applications the dispersion relation can be adapted in the following.

In 2007, the AB-equation, a new type of $\mathrm{KdV}$-equation, is derived in [46]. This equation is exact up to and including quadratic nonlinear terms and has exact dispersive properties. In [50], it is shown that the ABequation can accurately model waves in hydrodynamics laboratories that are generated by a flap motion. Moreover, unlike any other KdV-type of equation, this $\mathrm{AB}$-equation is valid and accurate for waves in deep water. By using Hamiltonian theory, it was shown that AB-equation approximates accurately the highest $120^{\circ}$ Stokes wave in deep water [50, 36]. Different from all steady traveling wave profiles which are smooth, the highest Stokes wave has a peak at the crest position and travel periodically with a constant speed $[6,36,37,50]$.

This paper is a continuation, and actually a combination, of these previous works. Our objective is to obtain an AB2-equation that deals just as KP with mainly uni-directional waves in two space dimensions, but shares the properties of the AB-equation of being accurate to second order in the wave height and having exact dispersion in the main direction of propagation. We will derive AB2 in a consistent way from the variational formulation of surface waves; in poorer approximations, $\mathrm{AB} 2$ will give various types of improved KP-type of equations.

Before dealing with the somewhat technical variational aspects, we will illustrate the basic result with a simple intuitive derivation of the classical $\mathrm{KP}$ and the new AB2-equation. 
We start with the simplest second order wave equation:

$$
\partial_{t}^{2} \eta=c_{0}^{2}\left(\partial_{x}^{2}+\partial_{y}^{2}\right) \eta
$$

For constant $c_{0}=\sqrt{g h}$ with $h$ the constant depth of the layer, this is a description of very long waves in the linear approximation. The dispersion relation is given by $\omega^{2}=c_{0}^{2}\left(k_{x}^{2}+k_{y}^{2}\right)$ which relates the frequency $\omega$ of harmonic waves to the wave numbers in the $x$ and $y$-direction. Purely unidirectional waves in the positive $x$-direction would be described by $\left(\partial_{t}+c_{0} \partial_{x}\right) \eta=$ 0 which has the dispersion relation $\omega=c_{0} k_{x}$. Multi-directional waves that mainly travel in the positive $x$-direction will have $\left|k_{y}\right|<<k_{x}$. This makes it tempting to approximate the second order spatial operator $c_{0}^{2}\left(\partial_{x}^{2}+\partial_{y}^{2}\right)$ via the dispersion relation like

$$
\begin{aligned}
\omega & =c_{0} k_{x} \sqrt{1+\left(k_{y} / k_{x}\right)^{2}} \\
& \approx c_{0} k_{x}\left(1+\frac{1}{2} \frac{k_{y}^{2}}{k_{x}^{2}}\right)=c_{0}\left(k_{x}+\frac{1}{2} \frac{k_{y}^{2}}{k_{x}}\right) .
\end{aligned}
$$

The corresponding equation is therefore $\partial_{t} \eta=-c_{0}\left(\partial_{x} \eta+\frac{1}{2} \partial_{x}^{-1} \partial_{y}^{2} \eta\right)$, which can be rewritten in a more appealing way like

$$
\partial_{x}\left[\partial_{t} \eta+c_{0} \partial_{x} \eta\right]+\frac{c_{0}}{2} \partial_{y}^{2} \eta=0
$$

This is the approximate equation for infinitesimal long waves traveling mainly in the positive $x$-direction. If we want to include approximate dispersion and nonlinearity in the $x$-direction only, the $\mathrm{KdV}$-equation could be taken as approximation: $\partial_{t} \eta+c_{0} \partial_{x} \eta+c_{0} \frac{h^{2}}{6} \partial_{x}^{3} \eta+\frac{3 c_{0}}{2 h} \eta \partial_{x} \eta=0$. Changing the term in brackets by this expression leads to the standard form of the KP-equation:

$$
\partial_{x}\left[\partial_{t} \eta+c_{0}\left(\partial_{x} \eta+\frac{h^{2}}{6} \partial_{x}^{3} \eta\right)+\frac{3 c_{0}}{2 h} \eta \partial_{x} \eta\right]+\frac{c_{0}}{2} \partial_{y}^{2} \eta=0 .
$$

A simple scaling can normalize all coefficients in the bracket in a frame moving with speed $c_{0}$. 
Improving on this heuristic derivation, we will show in this paper that the more accurate AB2-equation can be derived in a consistent way. A simplified form of AB2 could be obtained by replacing the term in square brackets in (2.3) by the AB-equation, and at the same time adding dispersive effects related to the group velocity in the term with the second order transversal derivatives that are consistently related to the dispersion within the square bracket. The most appealing equation is found that matches second order accuracy in wave height with second order transversal accuracy, to be called AB22, given by

$$
\partial_{x}\left[\partial_{t} \eta+\sqrt{g} A\left(\begin{array}{c}
\eta-\frac{1}{4}(A \eta)^{2}+\frac{1}{2} A(\eta A \eta) \\
+\frac{1}{4}(B \eta)^{2}+\frac{1}{2} B(\eta B \eta)
\end{array}\right)\right]+\frac{1}{2} V \partial_{y}^{2} \eta=0
$$

where the term in square brackets is the AB-equation of [46].

This paper will follow the consistent derivation for waves in one direction as given in [46]. The major difference is to account for small deviations in the unidirectionalization procedure; this requires some careful dealing with skew symmetric square-root operators.

The content of the paper is as follows. In the next section we describe the variational structure and the approximation of the action principle that will be used in the following sections. Section 3 discusses the linear 2dimensional wave equation and the unidirectional constraint to obtain an approximate linear 2D-equation that has exact dispersion in the main propagation direction. This will be the linearized AB2-equation which will be derived in Section 4, and shown to be accurate in second order of the wave height. In Section 5 some approximate cases are considered. Some conclusions and remarks are given in Section 6.

\section{$2.2 \quad$ Variational structure}

We consider surface waves on a layer of irrotational, inviscid and incompressible fluid that propagate in the $\mathbf{x}=(x, y)$ direction over a finite depth 
$h_{0}$ or over infinite depth. We denote the wave elevation by $\eta(\mathbf{x}, t)$ and the fluid potential by $\Phi(\mathbf{x}, z, t)$ with $\phi(\mathbf{x}, z=\eta, t)$ the fluid potential at the surface.

Based on previous work of $[29,61,5]$, the dynamic equations can be derived from variations of the action principle, i.e: $\delta A(\eta, \phi)=0$, where

$$
A(\eta, \phi)=\int\left[\int \phi . \partial_{t} \eta d \mathbf{x}-H(\eta, \phi)\right] d t .
$$

Variations with respect to $\phi$ and $\eta$ lead to the following coupled equations:

$$
\begin{gathered}
\partial_{t} \eta=\delta_{\phi} H(\eta, \phi), \\
\partial_{t} \phi=-\delta_{\eta} H(\eta, \phi) .
\end{gathered}
$$

The Hamiltonian $H(\eta, \phi)$ is the total energy, the sum of the potential and the kinetic energy, expressed in the variables $\eta, \phi$. The potential energy is calculated with respect to the undisturbed water level, leading to

$$
H(\eta, \phi)=\int \frac{1}{2} g \eta^{2} d \mathbf{x}+K(\phi, \eta) .
$$

The value of the kinetic energy is given by:

$$
K(\phi, \eta)=\iint \frac{1}{2}|\nabla \Phi|^{2} d z d \mathbf{x}
$$

where the fluid potential $\Phi$ satisfies the Laplace equation $\Delta \Phi=0$ in the fluid interior, the surface condition $\Phi=\phi$ at $z=\eta(\mathbf{x})$ and the impermeable bottom boundary condition. This kinetic energy cannot be expressed explicitly as a functional in terms of $\phi$ and $\eta$, and therefore approximations are needed. To that end, we will use the notation of the fluid potential and the vertical velocity at the still water as

$$
\phi_{0}=\Phi(\mathbf{x}, z=0, t), W_{0}=\partial_{z} \Phi(\mathbf{x}, z=0, t)
$$


respectively. To approximate the kinetic energy, we split the kinetic energy in an expression till the still water level and an additional term to account for the actual surface elevation:

$$
K=K_{0}+K_{s}=\iint_{z=-h}^{z=0} \frac{1}{2}|\nabla \Phi|^{2} d z d \mathbf{x}+\iint_{z=0}^{z=\eta} \frac{1}{2}|\nabla \Phi|^{2} d z d \mathbf{x} .
$$

The bulk kinetic energy $K_{0}$ can be rewritten using $\Delta \Phi=0$ and the divergence theorem with the permeability bottom condition as follows:

$$
K_{0}=\iint_{z=-h}^{z=0} \frac{1}{2} \nabla \cdot(\Phi \nabla \Phi) d z d \mathbf{x}=\int \frac{1}{2} \phi_{0} W_{0} d \mathbf{x} .
$$

Taking in $K_{s}$ the approximation of lowest order in the wave height, we obtain:

$$
\begin{aligned}
K_{s} & \approx \iint_{z=0}^{z=\eta}\left[\frac{1}{2}|\nabla \Phi|^{2}\right]_{z=0} d z d \mathbf{x} \\
& =\frac{1}{2} \int \eta\left[\left(\partial_{x} \phi_{0}\right)^{2}+\left(\partial_{y} \phi_{0}\right)^{2}+W_{0}^{2}\right] d \mathbf{x} .
\end{aligned}
$$

Taken together, we obtained the following approximation for the Hamiltonian:

$$
H\left(\eta, \phi_{0}\right) \approx \frac{1}{2} \int\left\{g \eta^{2}+\phi_{0} W_{0}+\eta\left[\left(\partial_{x} \phi_{0}\right)^{2}+\left(\partial_{y} \phi_{0}\right)^{2}+W_{0}^{2}\right]\right\} d \mathbf{x} .
$$

Observe that this expression is in the variables $\eta$ and $\phi_{0}$ (and $W_{0}$ which will be easily expressed in terms of $\phi_{0}$ in the next section). These are not the canonical variables, and hence $H\left(\eta, \phi_{0}\right)$ cannot be used in the Hamilton equations, except in the linear approximation of the next section. To include nonlinear effects, we will need to translate the pair $\eta, \phi_{0}$ to the canonical pair $\eta, \phi$ in Section 4. 


\subsection{Mainly unidirectional linear waves}

We start this section on linear wave theory with some notation to reduce possible confusion. For irrotational waves in one dimension, the dispersion relation between frequency $\omega$ and wavenumber $k$ is given by

$$
\omega^{2}=g k \tanh (k h) .
$$

By defining

$$
\Omega(k)=\operatorname{sign}(k) \sqrt{g k \tanh (k h)},
$$

the corresponding pseudo-differential operator $i \Omega\left(-i \partial_{x}\right)$ is skew-symmetric.

As usual we define the phase and group velocity respectively as

$$
C=\frac{\Omega}{k}, \text { and } V=\frac{d \Omega}{d k} ;
$$

we will use the same notation for the corresponding symmetric operators in the following.

Then the second order dispersive equation $\partial_{t}^{2} \eta=-\Omega^{2}\left(-i \partial_{x}\right) \eta=\partial_{x}^{2} C^{2} \eta$ can be written like

$$
\left(\partial_{t}^{2}+\Omega^{2}\right) \eta=\left(\partial_{t}-\partial_{x} C\right)\left(\partial_{t}+\partial_{x} C\right) \eta=0,
$$

showing that each solution is a combination of a wave running to the right, i.e. satisfying $\left(\partial_{t}+\partial_{x} C\right) \eta=0$, and a wave running to the left $\left(\partial_{t}-\partial_{x} C\right) \eta=0$.

For multi-directional waves, the dispersion relation is given with the wave vector $\mathbf{k}=\left(k_{x}, k_{y}\right)$ by

$$
\omega^{2}=g|\mathbf{k}| \tanh (|\mathbf{k}| h) .
$$

To deal with waves traveling mainly in the positive $x$-direction, we define

$$
\Omega_{2}(\mathbf{k})=\operatorname{sign}\left(k_{x}\right) \sqrt{g|\mathbf{k}| \tanh (|\mathbf{k}| h)} ;
$$

then, with $\nabla=\left(\partial_{x}, \partial_{y}\right)$, the operator $i \Omega_{2}(-i \nabla)$ is skew-symmetric. Note that $\Omega_{2}(\mathbf{k})$ is discontinuous for $k_{x}=0$ if $k_{y} \neq 0$, which will not happen when the assumption of mainly unidirectionality holds, since then $\left|k_{y}\right|<<k_{x}$. 
The phase velocity vector is given by

$$
C_{2}(\mathbf{k})=\Omega_{2}(\mathbf{k}) \frac{\mathbf{k}}{|\mathbf{k}|^{2}}=C(|\mathbf{k}|) \frac{\mathbf{k}}{|\mathbf{k}|} ;
$$

hence we have

$$
\Omega_{2}(\mathbf{k})=-i \alpha(\mathbf{k}) C(|\mathbf{k}|) \text {, with } \alpha(\mathbf{k})=i . \operatorname{sign}\left(k_{x}\right)|\mathbf{k}| .
$$

To make the analogy with the 1D case complete, we defined here the skew symmetric square root $\alpha$ operator of $-\nabla^{2}$; indeed, $\alpha=\partial_{x}$ if $\partial_{y}=0$, and $\alpha^{2}=\left(\partial_{x}^{2}+\partial_{y}^{2}\right)$ and $\alpha \alpha^{*}=-\left(\partial_{x}^{2}+\partial_{y}^{2}\right)$.

For later reference, we note that $\Omega_{2}$ has for each $k_{x} \neq 0$ the following expression as second order Taylor expansion in $k_{y}$ at 0 :

$$
\Omega_{2}(\mathbf{k}) \approx \Omega\left(k_{x}\right)+\frac{1}{2} \frac{k_{y}^{2}}{k_{x}} V\left(k_{x}\right) .
$$

Using the above notation, we recall that the solution of the Laplace problem for the fluid potential implies the following expression for the vertical velocity at the surface

$$
W_{0}=-\frac{1}{g} \Omega_{2}^{2} \phi_{0}=-\frac{1}{g} C^{2} \alpha^{2} \phi_{0} .
$$

This leads to the following expression for the quadratic part of the Hamiltonian:

$$
\begin{aligned}
H_{0}\left(\eta, \phi_{0}\right) & =\frac{1}{2} \int\left\{g \eta^{2}+\phi_{0} W_{0}\right\} d \mathbf{x} \\
& =\frac{1}{2} \int\left\{g \eta^{2}-\frac{1}{g} \phi_{0} \alpha^{2} C^{2} \phi_{0}\right\} d \mathbf{x}
\end{aligned}
$$

and the corresponding Hamilton equations are given by

$$
\partial_{t} \eta=\delta_{\phi} H_{0}(\eta, \phi)=-\frac{1}{g} \alpha^{2} C^{2} \phi_{0},
$$




$$
\partial_{t} \phi_{0}=-\delta_{\eta} H_{0}(\eta, \phi)=-g \eta,
$$

which are equivalent with the second order equation:

$$
\partial_{t}^{2} \eta=C^{2} \alpha^{2} \eta
$$

We notice that when $C$ is a constant, this equation is exactly the hyperbolic equation that describes two dimensional non-dispersive traveling waves as described in the introduction. This linear equation can be rewritten as the application of two first order linear equation:

$$
\left(\partial_{t}-C \alpha\right)\left(\partial_{t}+C \alpha\right) \eta=0 .
$$

Hence we find the dispersive equation for mainly uni-directional waves in the positive $x$-direction as

$$
\left(\partial_{t}+C \alpha\right) \eta=0
$$

As in [46], we will execute this 'unidirectionalization' procedure in another way via the variational principle. To illustrate that this leads to the correct result above, we note that the dynamic equation for $\phi_{0}$ leads for mainly unidirectional waves to a constraint between $\phi_{0}$ and $\eta$ given by $\partial_{t} \phi_{0}=-C \alpha \phi_{0}=-g \eta$, i.e. $\phi_{0}=g \alpha^{-1} C^{-1} \eta$.

Restricting the action functional to this constraint, leads to:

$$
\overline{\mathcal{A}_{0}}(\eta)=\int\left[\int g \alpha^{-1} C^{-1} \eta . \partial_{t} \eta d \mathbf{x}-\overline{H_{0}}(\eta)\right] d t,
$$

with the restricted Hamiltonian

$$
\begin{aligned}
\overline{H_{0}}(\eta) & =\frac{g}{2} \int \eta^{2}-\left(\partial_{x}^{2}+\partial_{y}^{2}\right)^{-1} \alpha \eta \cdot \alpha \eta d \mathbf{x} \\
& =g \int \eta^{2} d \mathbf{x}
\end{aligned}
$$


Notice that the kinetic energy and the potential energy functionals have the same value, i.e. equipartition of energy as is known to hold for linear wave evolutions.

The dynamic equation of $\eta$ then follows from the action principle, i.e. $\delta \overline{\mathcal{A}_{0}}(\eta)=0$, leading to:

$$
\partial_{t} \eta=-C \alpha \eta
$$

This is the linear equation for waves mainly running in the positive $x$ direction. Expansion of the operators to second order in $k_{y}$ using (2.10), it can be written like:

$$
\partial_{x}\left[\partial_{t} \eta+\partial_{x} C\left(-i \partial_{x}\right) \eta\right]+\frac{1}{2} V\left(-i \partial_{x}\right) \partial_{y}^{2} \eta=0 .
$$

This is recognized as an improvement of the linear KP-equation that is of the same second order in the tranversal direction. Observe, in particular, the appearance of the group-velocity operator in the last term, which is consistently linked to the (possibly approximated) phase velocity operator in the square brackets. When we use the approximation for rather long waves, as in the KP-equation, we have $C=c_{0}\left(1+\frac{h^{2}}{6} \partial_{x}^{2}\right)$ and then consistently $V=c_{0}\left(1+\frac{h^{2}}{2} \partial_{x}^{2}\right)$; this last operator is missing in the original KPequation.

We notice also that equation(2.16) is for purely unidirectional waves, $\partial_{y}=0$, the same as the linear part of AB-equation as derived in [46] .

\subsection{Nonlinear AB2-equation}

In the previous section, we used the quadratic part of the Hamiltonian and the unidirectionalization procedure in the action principle to obtain the linear AB2-equation. In this section, we will include the cubic terms of $\eta$ that appear in the approximation of the Hamiltonian(2.9). In the following, we use the same unidirectionalization constraint as describe above, i.e. taking $\phi_{0}=g C^{-1} \alpha^{-1} \eta$. For simplification, we introduce the following operators 


$$
A_{2}=\frac{C(-i \nabla) \alpha}{\sqrt{g}}, B_{2}=\partial_{x} A_{2}^{-1} \text { and } \gamma_{2}=\partial_{y} A_{2}^{-1},
$$

and rewrite the Hamiltonian (2.9) as

$$
\begin{aligned}
H\left(\eta, \phi_{0}\right) & =g \int\left\{\eta^{2}+\frac{1}{2} \eta\left[\left(A_{2} \eta\right)^{2}+\left(B_{2} \eta\right)^{2}+\left(\gamma_{2} \eta\right)^{2}\right]\right\} d \mathbf{x} \\
& =\left[H_{2}+H_{3}\right](\eta),
\end{aligned}
$$

with $H_{2}$ and $H_{3}$ the quadratic and the cubic terms of the Hamiltonian respectively.

For the nonlinear case considered here, we have to use the action principle with $\iint \phi \partial_{t} \eta d \mathbf{x} d t$ which contains the potential $\phi$ at the surface $z=\eta$. The strategy is to relate $\phi$ to $\phi_{0}, \eta$ by a direct expansion $\phi=\phi_{0}+\eta W_{0}$. Then using the unidirectionalization constraint $\phi_{0}=\sqrt{g} A_{2}^{-1} \eta$ and $W_{0}=$ $-\sqrt{g} A_{2} \eta$, we get

$$
\iint \phi \partial_{t} \eta d \mathbf{x} d t=\iint \sqrt{g}\left[A_{2}^{-1} \eta-\eta A_{2} \eta\right] \partial_{t} \eta d \mathbf{x} d t
$$

The action functional becomes

$$
\mathcal{A}(\eta)=\int\left\{\int \sqrt{g}\left[A_{2}^{-1} \eta-\eta A_{2} \eta\right] \partial_{t} \eta d \mathbf{x}-H(\eta)\right\} d t
$$

and vanishing of the variational derivative leads to the evolution equation

$$
\sqrt{g}\left[-2 A_{2}^{-1} \partial_{t} \eta+A_{2}\left(\eta \partial_{t} \eta\right)+\eta A_{2} \partial_{t} \eta\right]=\delta_{\eta} \bar{H}(\eta)
$$

with

$$
\begin{aligned}
\delta_{\eta} \bar{H}(\eta) & =g\left[2 \eta+\frac{1}{2}\left(A_{2} \eta\right)^{2}-A_{2}\left(\eta A_{2} \eta\right)\right. \\
& \left.+\frac{1}{2}\left(B_{2} \eta\right)^{2}+B_{2}\left(\eta B_{2} \eta\right)+\frac{1}{2}\left(\gamma_{2} \eta\right)^{2}+\gamma_{2}\left(\eta \gamma_{2} \eta\right)\right]
\end{aligned}
$$


Although this formulation is correct, the expression involving $\partial_{t} \eta$ is rather complicated. We will simplify it in the same way as in [46]. To that end, we note that

$$
-2 \sqrt{g} A_{2}^{-1} \partial_{t} \eta=2 g \eta+O\left(\eta^{2}\right), \text { i.e. : } \partial_{t} \eta=-\sqrt{g} A_{2} \eta+O\left(\eta^{2}\right),
$$

and substitute this approximation in the action to obtain

$$
\int \sqrt{g}\left[A_{2}^{-1} \eta-\eta A_{2} \eta\right] \partial_{t} \eta d \mathbf{x}=\int\left[\sqrt{g} A_{2}^{-1} \eta \partial_{t} \eta+g \eta\left(A_{2} \eta\right)^{2}\right] d \mathbf{x} .
$$

In this way, the total action functional is approximated correctly up to and including cubic terms in wave height. We write the result as a modified action principle, reading explicitly

$$
A_{\text {mod }}(\eta)=\int\left[\int \sqrt{g} A_{2}^{-1} \eta \partial_{t} \eta d \mathbf{x}-\bar{H}_{\text {mod }}(\eta)\right] d t
$$

where the modified Hamiltonian $\bar{H}_{\text {mod }}$ contains a term from the original action and is given by

$$
\begin{aligned}
\bar{H}_{\text {mod }}(\eta) & =\bar{H}-g \int \eta\left(A_{2} \eta\right)^{2} d \mathbf{x} \\
& =g \int \eta^{2}+\frac{1}{2} \eta\left\{-\left(A_{2} \eta\right)^{2}+\left(B_{2} \eta\right)^{2}+\left(\gamma_{2} \eta\right)^{2}\right\} d \mathbf{x} .
\end{aligned}
$$

The resulting equation $\delta A_{\bmod }(\eta)=0$ :

$$
-2 \sqrt{g} A_{2}^{-1} \partial_{t} \eta=\delta \bar{H}_{\text {mod }}(\eta)
$$

will be called the AB2-equation and it is explicitly given by:

$$
\partial_{t} \eta=-\sqrt{g} A_{2}\left[\begin{array}{c}
\eta-\frac{1}{4}\left(A_{2} \eta\right)^{2}+\frac{1}{2} A_{2}\left(\eta A_{2} \eta\right) \\
+\frac{1}{4}\left(B_{2} \eta\right)^{2}+\frac{1}{2} B_{2}\left(\eta B_{2} \eta\right) \\
+\frac{1}{4}\left(\gamma_{2} \eta\right)^{2}+\frac{1}{2} \gamma_{2}\left(\eta \gamma_{2} \eta\right)
\end{array}\right]
$$


From the derivation above, we notice that AB2-equation is exact up to and including the second order in the wave height, with (possible approximations of the) dispersion relation reflected in the operators $A_{2}, B_{2}$ and $\gamma_{2}$.

\subsection{Approximations of the AB2-equation}

In this section, we consider some special limiting cases of the AB2-equation.

If there is no dependence on the $y$-direction, i.e. waves travel uniformly in the positive $x$-direction, we can ignore the last two terms of equation (2.24) since $\gamma_{2}=0$, and the operators $\alpha, A_{2}$ and $B_{2}$ can be rewritten as follows:

$$
\alpha=\partial_{x}, A_{2}=A \equiv \frac{C\left(-i \partial_{x}\right)}{\sqrt{g}} \partial_{x} \text { and } B_{2}=B \equiv \partial_{x} A^{-1}
$$

Substituting these operators in equation(2.24), we obtain the original ABequation for the unidirectional wave as derived in [46]:

$$
\partial_{t} \eta=-\sqrt{g} A\left(\eta-\frac{1}{4}(A \eta)^{2}+\frac{1}{2} A(\eta A \eta)+\frac{1}{4}(B \eta)^{2}+\frac{1}{2} B(\eta B \eta)\right) .
$$

This equation is exact in the dispersion relation and is second order accurate in the wave height. It is an improvement of the $\mathrm{KdV}$-equation for waves above finite depth. For infinite depth, this is a new equation in which all four quadratic terms are of the same order.

In section 3 we investigated the approximation of the exact linear equation to second order in $k_{y}$, which leads to the improvement of the linear KPequation but retains its characteristic form. Now we will make a similar approximation for the nonlinear AB2-equation. To keep the basic variational structure, any approximation can be obtained by taking approximations of the operators $A_{2}$ and the consistent approximations for $B_{2}=\partial_{x} A_{2}^{-1}$ and $\gamma_{2}=\partial_{y} A_{2}^{-1}$. 
Here we will consider only one special case, namely an approximation for which we neglect tranversal effects in the nonlinear terms. Stated differently, we approximate equation(2.24) such that second order nonlinear and transversal effects are considered to be of the same order. Using the operators $A, B$ as above, we obtain the AB22-equation (2.4) mentioned in the introduction.

It should be remarked that in all the results above we did not restrict the wavelength in the $x$-direction; all results are valid also for short waves in the main propagation direction, the dispersion properties in the $x$-direction are exact. Also, note that, just like $\mathrm{KdV}$, the $\mathrm{KP}$ - equation has no sensible limit for infinite depth, but that, just like the AB-equation, the AB2- and AB22-equation, are also valid for deep water waves.

\subsection{Conclusion and remarks}

The KP-equation as a model for mainly unidirectional surface water waves has been improved in this paper to the AB2-equation that has exact dispersion and is exact up to and including second order in the wave height. As was found for the unidirectional version of this equation, the AB-equation, we expect that with AB2 very accurate simulations can be performed. In a subsequent paper we will show results of comparisons with measurements in a hydrodynamic laboratory. The most difficult aspect to predict beforehand is the practical validity in the transversal direction, i.e. what the maximal deviation from the main propagation direction can be so that the waves are still accurately modeled. 


\section{Chapter 3}

\section{Embedded wave generation for dispersive wave models ${ }^{1}$}

\subsection{Introduction}

Wave models of Boussinesq type for the evolution of surface waves on a layer of fluid describe the evolution with quantities at the free surface. These models have dispersive properties that are directly related to the -unavoidable- approximation of the interior fluid motion. The initial value problem for such models does not cause much problems, since the description of the state variables in the spatial domain at an initial instant is independent of the specifics of the evolution model.

Quite different is the situation when waves have to be excited in a timely manner from points or lines. Such problems arise naturally when modelling waves in a hydrodynamic laboratory or waves from the deep ocean to a coastal area. In these cases the waves can be generated by influx-boundary conditions, or by some embedded, internal, forcing. In all cases the dispersive properties (of the implementation) of the model are present in the

\footnotetext{
${ }^{1}$ Part of this chapter (until section 3.2.2) is similar to a paper that has been submitted for publication ( Lie S.L, D. Adytia \& E. van Groesen. Embedded wave generation for dispersive surface wave models. Ocean Engineering, 2013.)
} 
details of the generation. Accurate generation is essential for good simulations, since slight errors will lead after propagation over large distances to large errors. For various Boussinesq type equations, internal wave generation has been discussed in several papers. Improving the approach of Engquist \& Madja [12], who described the way how to influx waves at the boundary with the phase speed, Wei e.a. in [55] considered the problem to generate waves from the $y$-axis under an angle $\theta$ with respect to the positive $x$-axis. They derived in an analytical way a spatially distributed source function method for the Boussinesq model of Wei \& Kirby [54] that is based on a spatially distributed source, with an explicit relation between the desired surface wave and the source function. Kim e.a. [20] showed for various Boussinesq models that it is possible to generate oblique waves using a delta source function. Madsen \& Sorensen [30] used and formulated a source function for mild slope equations. In these papers, the results were derived for the linearized equations.

In this chapter we derive source functions for any kind of waves to be generated and for any dispersive equation including the general case of (linear) dispersive Boussinesq equations. Consequently, the results are applicable for the equations considered in the references mentioned above, such as Boussinesq equations of Peregrine [9], the extended Boussinesq equations of Nwogu [34] and those of Madsen \& Sorensen [30], and for the mild slope equations of Massel [32], Suh et al. [44] and Lee et al. [25, 26]. In $[48,50]$ the method to be described here for the AB-equation has been used and also in [3, 23] for the Variational Boussinesq model.

We will derive the wave generation approach in a straightforward and constructive way for linear equations. The group velocity derived from the specific dispersion relation will turn up in the various choices that can be made for the non-unique source function. We will show that the linear generation approach is accurate through various examples in $1 \mathrm{D}$ and $2 \mathrm{D}$.

This chapter is organized as follows. In the next section we present the wave generation both in $1 \mathrm{D}$ and $2 \mathrm{D}$ for forward propagation wave equations with arbitrary dispersive properties. The wave generation for multidirectional wave equations is presented in section 3. Simulation results will be shown in section 4 , and the chapter is finished with conclusions. 


\subsection{Forward propagating dispersive wave models}

\subsubsection{Definitions and notation}

In this section we consider $1 \mathrm{D}$ and $2 \mathrm{D}$ forward propagating wave models. To deal with both cases at the same time, we use the following notation. For $1 \mathrm{D}$ we use $x$ as spatial coordinate, and $k$ for the wave number. In $2 \mathrm{D}$ we use coordinates $\mathbf{x}=(x, y)$ and wave vector $\mathbf{k}=\left(k_{x}, k_{y}\right)$, and write for the lengths of these vectors $x=|\mathbf{x}|$ and $k=|\mathbf{k}|$ respectively.

In $1 \mathrm{D}$ we denote by $\eta(x)$ the wave elevation and use the convention that $\eta(x)$ and its (spatial) Fourier transformation $\hat{\eta}(k)$ are related to each other by

$$
\eta(x)=\int \hat{\eta}(k) e^{i k x} d k, \quad \hat{\eta}(k)=\frac{1}{2 \pi} \int \eta(x) e^{-i k x} d x .
$$

Similarly for the $2 \mathrm{D}$ case we have

$$
\eta(\mathbf{x})=\int \hat{\eta}(\mathbf{k}) e^{i \mathbf{k} \cdot \mathbf{x}} d \mathbf{k}, \quad \hat{\eta}(\mathbf{k})=\frac{1}{(2 \pi)^{2}} \int \eta(\mathbf{x}) e^{-i \mathbf{k} \cdot \mathbf{x}} d \mathbf{x} .
$$

When it is not indicated otherwise, integrals are taken over the whole real axis. To describe real waves in the following, the condition

$$
\hat{\eta}(-\mathbf{k})=c c(\hat{\eta}(\mathbf{k}))
$$

will have to be satisfied for each wave number or wave vector, where $c c$ denotes complex conjugation.

Dispersion is the property that for plane waves the wave length and period are not independent but related in a specific way. That is, a dispersion relation between the wave number $k$ (in $1 \mathrm{D}$ ) or the wave vector $\mathbf{k}$ (in $2 \mathrm{D}$ ) and the frequency $\omega$ should be satisfied so that the harmonic mode in $1 \mathrm{D}$ $\exp i(k x-\omega t)$ or the plane wave in $2 \mathrm{D} \exp i(\mathbf{k} \cdot \mathbf{x}-\omega t)$ are physical solutions. For small amplitude water waves, i.e. linear theory, the quadratic dispersion relation is known to be given by

$$
\omega^{2}=\Omega^{2}(k)
$$


where we define the function $\Omega$ as

$$
\Omega(k)=\sqrt{g k \tanh (k h)}
$$

with $g$ and $h$ the gravitational acceleration and depth of the fluid layer respectively.

In order to work conveniently with complex notation and Fourier transformation in the following, we will consider the uni-directional dispersion relation using the (smooth) odd function $\Omega_{1}(k)$

$$
\omega=\Omega_{1}(k), \text { with } \Omega_{1}(k)=\operatorname{sign}(k) \Omega(k)
$$

With this convention, the wave $\exp i\left(k x-\Omega_{1}(k) t\right)=\exp i k(x-C(k) t)$ is for all values of $k$ to the right travelling with positive phase speed $C(k)=$ $\Omega_{1}(k) / k$; similarly $\exp i\left(k x+\Omega_{1}(k) t\right)$ is to the left travelling with speed $-C(k)$. Besides that, the relation has a unique inverse which we will denote by $K_{1}$ :

$$
\omega=\Omega_{1}(k) \Leftrightarrow k=K_{1}(\omega) .
$$

In a similar way we can talk for plane waves in $2 \mathrm{D}$ about forward and backward propagating with respect to some chosen direction e, given by $\exp i\left(\mathbf{k} \cdot \mathbf{x}-\Omega_{2}(\mathbf{k}) t\right)$ and $\exp i\left(\mathbf{k} \cdot \mathbf{x}+\Omega_{2}(\mathbf{k}) t\right)$ respectively, by defining

$$
\omega=\Omega_{2}(\mathbf{k}), \text { with } \Omega_{2}(\mathbf{k})=\operatorname{sign}(\mathbf{k} \cdot \mathbf{e}) \Omega(k)
$$

For later reference, we define the group velocity as the even function

$$
V(k)=\frac{d \Omega_{1}(k)}{d k} .
$$

The exact dispersion given above corresponds to a monotone concave relation of $\omega$ versus $k$, so that the phase velocity decreases for shorter waves. Observe that $\Omega_{1}$ scales with depth like

$$
\Omega_{1}(k)=\frac{\sqrt{g}}{\sqrt{h}} M(k h)
$$


and the group velocity as

$$
V_{g}(k, h)=c_{0} m(k h) \text { with } c_{0}=\sqrt{g h}
$$

where $m$ is the derivative of $M$.

In many models to be used for analytic or numerical investigations, an approximation of the exact dispersion relation is taken; all good approximations will satisfy the same scaling properties. As one example we mention the Variational Boussinesq model (VBM) described in [22]. In that model, the dependence of the fluid potential in the vertical direction $z$ is prescribed by an a priori chosen function $F(z)$. The dispersion relation then reads

$$
\Omega_{V B M}(k)=c_{0} k \sqrt{1-\frac{(k \beta)^{2}}{h\left(\alpha k^{2}+\mu\right)}},
$$

where $\alpha, \beta$ and $\mu$ are coefficients given by

$$
\alpha=\int_{-h}^{0} F(z)^{2} d z ; \beta=\int_{-h}^{0} F(z) d z ; \mu=\int_{-h}^{0}\left(\partial_{z} F(z)\right)^{2} d z .
$$

A flexible choice for $F(z)$ is to take the following explicit function

$$
F(z)=\frac{\cosh (\kappa(z+h))}{\cosh (\kappa h)}-1
$$

where $\kappa$ is a suitable effective wave number.

For shallow water the long wave approximation has dispersion relation given as $\Omega_{S W}=c_{0} k$. In figure (3.1) we show the plot of the exact dispersion relation and the exact group velocity together with the approximations described above.

In the following we will regularly need the spatial inverse Fourier transform of the group velocity, defined with a scaling factor as

$$
\gamma(x ; h)=\int \frac{1}{2 \pi} V_{g}(k, h) e^{i k x} d k .
$$



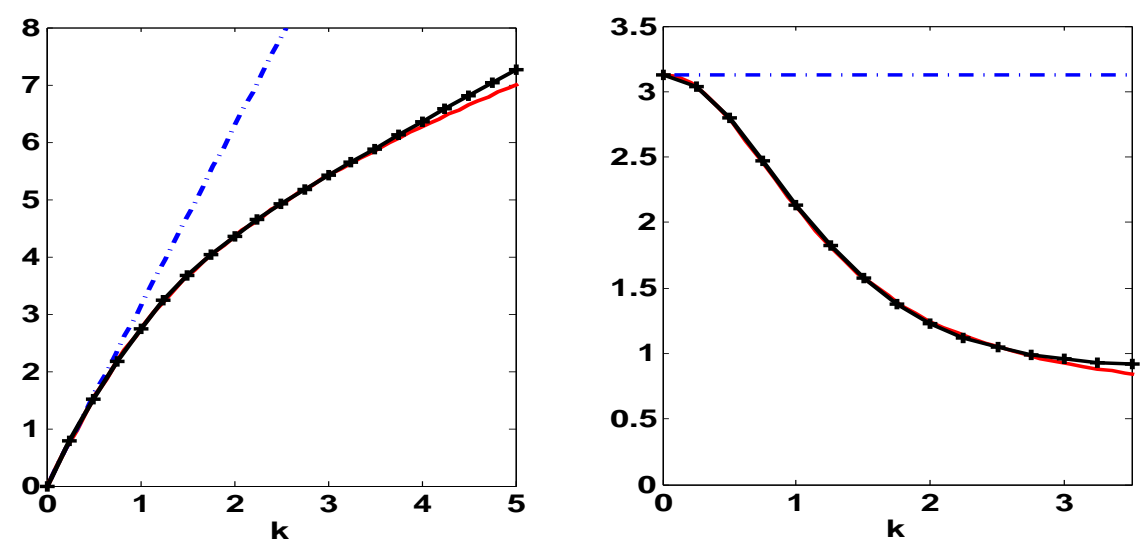

Figure 3.1: Plot of the dispersion relation (left panel) and the group velocity (right panel) as function of wave number for depth $1[\mathrm{~m}]$. The solid curve is the exact dispersion and group velocity; the dash-dotted and cross-dotted curves are the approximation for shallow water and VBM (with $\kappa=0.52$ )respectively.

The scaling property of the group velocity implies that its spatial inverse Fourier transform $\gamma(x ; h)$ scales with depth like

$$
\gamma(x ; h)=\frac{\gamma(x / h ; 1)}{\sqrt{h}} .
$$

The graph of this function $\gamma$ is given in figure (3.2) for the dispersion relations discussed above. For increasing depth, the function decreases proportional to $1 / \sqrt{h}$, the spatial extent of the function $\gamma$ grows proportional with $h$ and the area under the curve grows with $\sqrt{h}$ since $\int \gamma(x ; h) d x=$ $\sqrt{h} \int \gamma(x ; 1) d x$.

\subsubsection{D uni-directional waves}

The first order, 1D uni-directional equation for to the right (positive $x$-axis) traveling waves is of the form

$$
\partial_{t} \eta=-\mathcal{A}_{1} \eta
$$



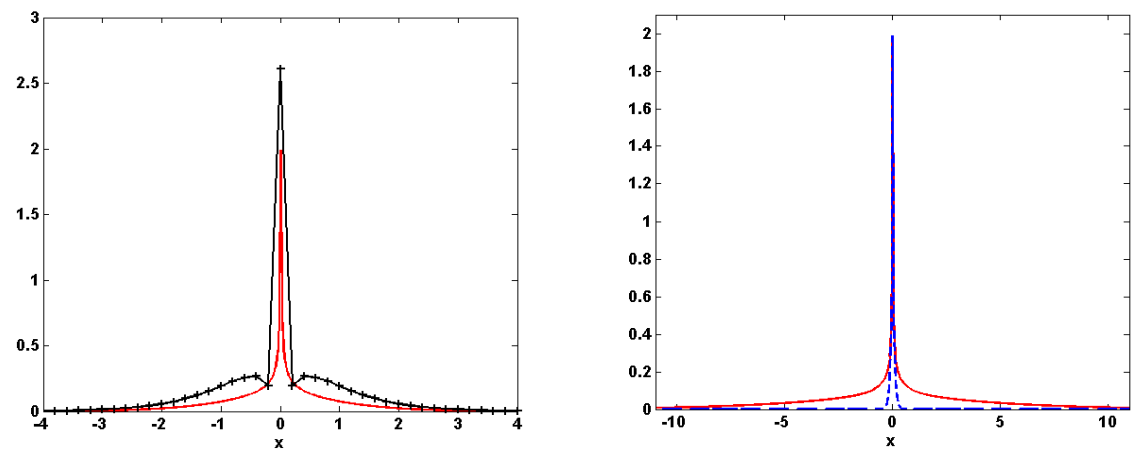

Figure 3.2: The left panel is the graph of $\gamma(x)$ at depth $h=1[m]$ for the exact dispersion-relation (solid line) and the approximate dispersion relations of VBM (cross-dotted line). The right panel is the graph of $\gamma(x)$ for exact dispersion on a depth of $h=5[\mathrm{~m}]$ (solid line) and $h=0.1[\mathrm{~m}]$ (dotted line). Observed that the shallower the water the closer the function resembles the Dirac delta function.

Here $\mathcal{A}_{1}$ is the (pseudo-differential) operator that has as symbol the dispersion relation $\Omega_{1}$, written as

$$
\mathcal{A}_{1} \hat{=} i \Omega_{1}(k)
$$

meaning that the effect of $\mathcal{A}_{1}$ applied to a function $\eta$ is corresponds to multiplication in spectral space by $i \Omega_{1}(k)$, i.e.

$$
\mathcal{A}_{1} \eta(x)=i \int \Omega_{1}(k) \hat{\eta}(k) e^{i k x} d k .
$$

The factor $\operatorname{sign}(k)$ assures that the real function $\Omega_{1}(k)$ is odd, which implies that $\mathcal{A}_{1}$ is a real operator that is skew-symmetric.

In the shallow water limit the dispersion relation is $\Omega_{1}(k)=c_{0} k$ with $c_{0}=\sqrt{g h}$, which corresponds to $\mathcal{A}_{1}=c_{0} \partial_{x}$, and the equation becomes $\partial_{t} \eta=-c_{0} \partial_{x} \eta$. Although this limiting case is not dispersive since all modes travel at the same speed $c_{0}$, it illustrates the uni-directionality property of the equation: all solutions are of the form $G\left(x-c_{0} t\right)$ for arbitrary $G$. 
The signalling problem for this linear dispersive model is formulated for the surface elevation $\zeta=\zeta(x, t)$ as

$$
\left\{\begin{array}{l}
\partial_{t} \zeta=-\mathcal{A}_{1} \zeta \\
\zeta(0, t)=s(t)
\end{array}\right.
$$

At one position, taken without restriction of generality to be $x=0$, the surface elevation is prescribed by the signal $s(t)$. Here and in the following we will assume the initial surface elevation and the signal to vanish for negative time: $\zeta(x, 0)=0$ and $s(t)=0$ for $t \leq 0$.

For the temporal Fourier transform $\check{s}(\omega)$ of the signal $s(t)$ we use the convention

$$
s(t)=\int \check{s}(\omega) e^{-i \omega t} d \omega \text { and } \check{s}(\omega)=\frac{1}{2 \pi} \int s(t) e^{i \omega t} d t .
$$

Then the solution of the signaling problem can be written explicitly as

$$
\zeta(x, t)=H(x) \int \check{s}(\omega) e^{i\left[K_{1}(\omega) x-\omega t\right]} d \omega,
$$

with $H(x)$ the Heaviside function. By rewriting this expression such that $s(t)$ appears explicitly, we get

$$
\zeta(x, t)=\frac{1}{2 \pi} H(x) \iint s(\tau) e^{i\left[K_{1}(\omega) x-\omega(t-\tau)\right]} d \omega d \tau .
$$

Note that, as a consequence of the fact that the integration over $\tau$ extends to infinity, this exact solution is for genuine dispersive equations non-causal: the solution $\zeta(x, t)$ at time $t$ depends also on the influx signal $s(\tau)$ for times $\tau>t$, although these contributions are exponentially small. An exception is when the dispersion relation is linear $\Omega_{1}(k)=c_{0} k$ in the case of the shallow water equation; then this integral expression simplifies to the correct solution $\zeta(x, t)=H(x) s\left(t-x / c_{0}\right)$.

If the influx point $x=0$ is a boundary of the spatial interval, the desired influx can be dealt with in numerical models as a boundary condition. Different from that way of influxing, in this paper we will produce the 
solution of the signaling problem by describing the influx in an embedded way. That is, we will investigate the forced problem of the form

$$
\partial_{t} \eta=-\mathcal{A}_{1} \eta+S_{1}(x, t)
$$

where we will look for embedded source(s) $S_{1}(x, t)$ in such a way that the source contributes to the elevation at $x=0$ by an amount determined by the prescribed signal $s(t)$. For the first order, uni-directional equation we consider, we expect a unique solution; but, as will turn out, the source function will not be unique. The ambiguity is caused by the dependence of the source on the two indepedent variables $x$ and $t$. Once we prescribe the dependence on one variable, for instance a localised force that acts only at the point $x=0$, the source will be uniquely defined by the signal. The ambiguity can be exploited to satisfy additional requirements.

To obtain the condition for the source, we consider the double temporalspatial Fourier transform (to be denoted by a bar) of equation (3.3). Then with

$$
\eta(x, t)=\iint \bar{\eta}(k, \omega) e^{i(k x-\omega t)} d k d \omega,
$$

the result is

$$
-i \omega \bar{\eta}(k, \omega)=-i \Omega_{1}(k) \bar{\eta}(k, \omega)+\bar{S}_{1}(k, \omega) .
$$

Note that for $S_{1}=0$ we get the correct requirement that the dispersion relation $\omega=\Omega_{1}(k)$ should be satisfied. The forced equation has as solution

$$
\bar{\eta}(k, \omega)=\frac{\bar{S}_{1}(k, \omega)}{i\left(\Omega_{1}(k)-\omega\right)}
$$

which reads in physical space

$$
\eta(x, t)=\iint \frac{\bar{S}_{1}(k, \omega)}{i\left(\Omega_{1}(k)-\omega\right)} e^{i(k x-\omega t)} d k d \omega .
$$

Specified for $x=0$ we get the condition for the source:

$$
s(t)=\iint \frac{\bar{S}_{1}(k, \omega)}{i\left(\Omega_{1}(k)-\omega\right)} e^{-i \omega t} d k d \omega,
$$


or equivalently

$$
\check{s}(\omega)=\int \frac{\bar{S}_{1}(k, \omega)}{i\left(\Omega_{1}(k)-\omega\right)} d k .
$$

Using the fact that the dispersion relation is invertible, we make a change of variables, from $k$ to $\nu$ with $\nu=\Omega_{1}(k)$. Using the group velocity $V_{g}(k)$ and the inverse $K_{1}(\nu)$ such that $\nu=\Omega_{1}\left(K_{1}(\nu)\right)$ we get $d \nu=V_{g}\left(K_{1}(\nu)\right) d k$, and hence

$$
\check{s}(\omega)=\int \frac{\bar{S}_{1}\left(K_{1}(\nu), \omega\right)}{V_{g}\left(K_{1}(\nu)\right)} \frac{d \nu}{i(\nu-\omega)},
$$

Assuming $\bar{S}_{1}\left(K_{1}(\nu), \omega\right) / V_{g}\left(K_{1}(\nu)\right)$ to be an analytic function in the complex $\nu$-plane, Cauchy's principal value theorem leads to the result that

$$
\check{s}(\omega)=2 \pi \frac{\bar{S}_{1}\left(K_{1}(\omega), \omega\right)}{V_{g}\left(K_{1}(\omega)\right)} .
$$

and hence

$$
\bar{S}_{1}\left(K_{1}(\omega), \omega\right)=\frac{1}{2 \pi} V_{g}\left(K_{1}(\omega)\right) \check{s}(\omega) .
$$

This is the source condition, the condition that $S_{1}$ produces the desired elevation $s(t)$ at $x=0$. This condition shows that the function $\omega \rightarrow$ $\bar{S}_{1}\left(K_{1}(\omega), \omega\right)$ is uniquely determined by the given time signal. However, the function $\bar{S}_{1}(k, \omega)$ of 2 independent variables is not uniquely determined; it is only uniquely defined for points $(k, \omega)$ that satisfy the dispersion relation. Consequently, the source function $S(x, t)$ is not uniquely defined, and the spatial dependence can be changed when combined with specific changes in the time dependence, as stated above.

To illustrate this, and to obtain some typical and practical results, consider sources of the form

$$
S_{1}(x, t)=g(x) f(t)
$$

in which space and time are separated: $g$ describes the spatial extent of the source, and $f$ is the so-called modified influx signal. Then we have 
$\bar{S}_{1}(k, \omega)=\hat{g}(k) \check{f}(\omega)$ and get a combined condition for the functions $f$ and $g$ as follows

$$
\hat{g}\left(K_{1}(\omega)\right) \check{f}(\omega)=\frac{1}{2 \pi} V_{g}\left(K_{1}(\omega)\right) \check{s}(\omega) .
$$

Clearly, the functions $f$ and $g$ are not unique, which is illustrated for two special cases.

Point generation: For a source that is concentrated at $x=0$ we take $S_{1}(x, t)=\delta_{\text {Dirac }}(x) f(t)$, where here and in the following $\delta_{\text {Dirac }}(x)$ is the Dirac delta-function. Then $\hat{g}(k)=1 / 2 \pi$ and $\bar{S}_{1}(k, \omega)=\check{f}(\omega) / 2 \pi$ and we get the modified influx signal the function $f(t)$ from the source condition, leading to

$$
S_{1}(x, t)=\delta_{\text {Dirac }}(x) f(t) \text { with } \check{f}(\omega)=V_{g}\left(K_{1}(\omega)\right) \check{s}(\omega) .
$$

Observe that in physical space, the modified signal $f(t)$ is the convolution between the original signal $s(t)$ and the inverse temporal Fourier transform of the group velocity $\omega \rightarrow V_{g}\left(K_{1}(\omega)\right)$.

Area extended generation: A more general choice of the spatial extent of the source, given by a function $g(x)$, requires that we modify the influx signal according to

$$
\check{f}(\omega)=\frac{1}{2 \pi} \frac{V_{g}\left(K_{1}(\omega)\right)}{\hat{g}\left(K_{1}(\omega)\right)} \check{s}(\omega) .
$$

In particular, it is possible to influx the original signal i.e. $f(t)=s(t)$ provided we choose $\hat{g}(k)=\frac{1}{2 \pi} V_{g}(k)$, so that

$$
S_{1}(x, t)=\gamma(x) s(t) \text { with } \gamma(x)=\int \frac{1}{2 \pi} V_{g}(k) e^{i k x} d k .
$$

The scaling properties of this function $\gamma$ have been described in the previous section; since its extent will become large for deep water, this may not be a desirable choice. A smooth alternative would be to take a Gaussian profile such as $g(x)=\exp \left(-x^{2} / \beta\right)$ where the parameter $\beta$ can control the practical extent of the source area, as has been used by Wei e.a. [55] 
As a final remark, notice that the area extended and the point generation are the same for the case of the non-dispersive shallow water limit for which $\Omega_{1}(k)=c_{0} k$ and $V_{g}(k)=c_{0}$ (which then coincides with the phase velocity). In that case we have $\bar{S}_{1}\left(K_{1}(\omega), \omega\right)=c_{0} \check{s}(\omega) / 2 \pi$, and obtain the familiar result for influxing of a signal $s(t)$ at $x=0$ :

$$
\partial_{t} \eta=-c_{0} \partial_{x} \eta+c_{0} \delta_{\text {Dirac }}(x) s(t) .
$$

\subsubsection{D forward dispersive wave model}

In a similar way as we did in $1 \mathrm{D}$, we will define a skew symmetric operator $\mathcal{A}_{\mathrm{e}}$ for given direction vector $\mathrm{e}$ to formulate first order dynamic equations that describe waves propagation forward or backward with respect to the direction given by the vector e. Forward propagating wave modes have wavevector in the half space $\{\mathbf{k} \mid \mathbf{k} . \mathbf{e}>0\}$ and backward propagating modes in $\{\mathbf{k} \mid \mathbf{k} \cdot \mathbf{e}<0\}$. First order in time equations for forward or backward travelling waves are most useful for wave influxing in a specific part of a half plane, for instance when waves are generated in a hydrodynamic laboratory, or when dealing with coastal waves from the deep ocean towards the shore.

We describe the first order in time equations with an operator $\mathcal{A}_{\mathbf{e}}$ in analogy with the operator for $1 \mathrm{D}$ equations. Hence we define $\mathcal{A}_{\mathbf{e}}$ as the pseudo-differential operator that acts in Fourier space as multiplication:

$$
\mathcal{A}_{\mathbf{e}} \hat{=} i \Omega_{2}(\mathbf{k}) \text { with } \Omega_{2}(\mathbf{k})=\operatorname{sign}(\mathbf{k} . \mathbf{e}) \Omega(k) .
$$

Then $\Omega_{2}(\mathbf{k})=-\Omega_{2}(-\mathbf{k})$ and $\mathcal{A}_{\mathbf{e}}$ is a real, skew symmetric operator. Observe that $\Omega_{2}$ has discontinuity along the direction $\mathbf{e}^{\perp}$ (perpendicular to $\mathbf{e}$ ). The $2 \mathrm{D}$ forward propagating dispersive wave equation is then given as

$$
\partial_{t} \zeta=-\mathcal{A}_{\mathbf{e}} \zeta
$$

which has as basic solutions the plane waves $\exp i\left(\mathbf{k} \cdot \mathbf{x}-\Omega_{2}(\mathbf{k}) t\right)$. Without restriction of generality we will take in the following $\mathbf{e}=(1,0)$ so that $\Omega_{2}(\mathbf{k})=\operatorname{sign}\left(k_{x}\right) \Omega(k)$, where $k=\sqrt{k_{x}^{2}+k_{y}^{2}}$. 
For the 2D excitation problem, we consider influxing along the $y$-axis:

$$
\left\{\begin{array}{l}
\partial_{t} \eta=-\mathcal{A}_{\mathbf{e}} \eta+S_{2}(\mathbf{x}, t) \\
\eta(0, y, t)=s(y, t)
\end{array}\right.
$$

with $S_{2}(\mathbf{x}, t)$ is to be determined. Applying the same technique as in the $1 \mathrm{D}$ case, we obtain the requirement for $S_{2}(\mathbf{x}, t)$ to be satisfied:

$$
s(y, t)=\iint \frac{\bar{S}_{2}\left(k_{x}, k_{y}, \omega\right)}{i\left(\Omega_{2}\left(k_{x}, k_{y}\right)-\omega\right)} e^{i\left(k_{y} y-\omega t\right)} \mathbf{d k} d \omega
$$

or equivalently

$$
\check{s}\left(k_{y}, \omega\right)=\int \frac{\bar{S}_{2}\left(k_{x}, k_{y}, \omega\right)}{i\left(\Omega_{2}\left(k_{x}, k_{y}\right)-\omega\right)} d k_{x} .
$$

Now we make a change of integration variable from $k_{x}$ to $\nu=\Omega_{2}\left(k_{x}, k_{y}\right)$, which is possible because of the monotony of $\Omega_{2}$ with respect to $k_{x}$ at fixed $k_{y}$, leading to $k_{x}=K_{x}\left(k_{y}, \nu\right)$. Writing $K\left(k_{y}, \nu\right)=\sqrt{K_{x}^{2}+k_{y}^{2}}$ and using $d \nu / d k_{x}=\operatorname{sign}\left(k_{x}\right) \partial \Omega_{1} / \partial k . \partial k / \partial k_{x}=V_{g}(k)\left|k_{x}\right| / k$ we get :

$$
\check{s}\left(k_{y}, \omega\right)=\int \frac{\bar{S}_{2}\left(K_{x}\left(k_{y}, \nu\right), k_{y}, \omega\right)}{i(\nu-\omega)} \frac{K\left(k_{y}, \nu\right)}{\left|K_{x}\left(k_{y}, \nu\right)\right| V_{g}\left(K\left(k_{y}, \nu\right)\right)} d \nu .
$$

With Cauchy's integral theorem we obtain as condition for the source function

$$
\bar{S}_{2}\left(K_{x}\left(k_{y}, \omega\right), k_{y}, \omega\right)=\frac{1}{2 \pi} V_{g}\left(K\left(k_{y}, \omega\right) \frac{\left|K_{x}\left(k_{y}, \omega\right)\right|}{K\left(k_{y}, \omega\right)} \check{s}\left(k_{y}, \omega\right) .\right.
$$

If we write $S_{2}(x, y, t)=g(x) . f(y, t)$ or $\bar{S}_{2}\left(k_{x}, k_{y}, \omega\right)=\hat{g}\left(k_{x}\right) \check{f}\left(k_{y}, \omega\right)$ then for a given function $g(x)$ the function $f(y, t)$ should be chosen as the inverse Fourier transform of

$$
\check{f}\left(k_{y}, \omega\right)=\frac{1}{2 \pi} \frac{V_{g}\left(K\left(k_{y}, \omega\right)\right)}{\hat{g}\left(K_{x}\left(k_{y}, \omega\right)\right)} \frac{\left|K_{x}\left(k_{y}, \omega\right)\right|}{K\left(k_{y}, \omega\right)} \check{s}\left(k_{y}, \omega\right) .
$$


Limiting cases We consider 3 limiting cases as special consequences of the forcing equation given above.

1. Uniform influxing: If the prescribed signal is homogeneous along the $y$-axis, i.e. $s(y, t)=s_{1}(t)$, then $\check{s}\left(k_{y}, \omega\right)=\delta_{\text {Dirac }}\left(k_{y}\right) \check{s}_{1}(\omega)$, and this leads to:

$$
\check{f}\left(k_{y}, \omega\right)=\frac{\delta_{\text {Dirac }}\left(k_{y}\right)}{2 \pi} \check{s}_{1}(\omega) \frac{V_{g}(K(0, \omega))}{\hat{g}\left(K_{x}(0, \omega)\right)} \frac{\left|K_{x}(0, \omega)\right|}{K(0, \omega)} .
$$

Since now $\left|K_{x}(0, \omega)\right|=K(0, \omega)$ and $K_{x}(0, \omega)=K_{1}(\omega)$, we get

$$
\check{f}\left(k_{y}, \omega\right)=\frac{\delta_{\text {Dirac }}\left(k_{y}\right)}{2 \pi} \check{s}_{1}(\omega) \frac{V_{g}\left(K_{1}(\omega)\right)}{\hat{g}\left(K_{1}(\omega)\right)},
$$

which is the result as can be expected from the $1 \mathrm{D}$ case, see equation $(3.9)$.

2. Oblique wave generation: If the excitation signal at the $y$-axis is given as $s(y, t)=a e^{i\left(k_{y}^{0} y-\omega_{0} t\right)}$ with $k_{y}^{0}=k_{0} \sin \left(\theta_{0}\right)$ and $k_{0}$ is the wavenumber corresponding to $\omega_{0}$ and without loss of generality we assume $\omega_{0}>0$, then equation (3.13) becomes

$$
\check{f}\left(k_{y}, \omega\right)=\frac{1}{2 \pi} \delta\left(k_{y}-k_{y}^{0}\right) \delta\left(\omega-\omega_{0}\right) \frac{V_{g}\left(K\left(k_{y}, \omega\right)\right)}{\hat{g}\left(K_{x}\left(k_{y}, \omega\right)\right)} \frac{\left|K_{x}\left(k_{y}, \omega\right)\right|}{K\left(k_{y}, \omega\right)} .
$$

Transforming to the physical space the forcing equation is then given as :

$$
\begin{aligned}
S_{2}(x, y, t) & =g(x) a e^{i\left(k_{y}^{0} y-\omega_{0} t\right)} \frac{1}{2 \pi} \frac{V_{g}\left(K_{1}\left(\omega_{0}\right)\right)}{\hat{g}\left(k_{x}^{0}\right)} \frac{k_{x}^{0}}{K_{1}\left(\omega_{0}\right)} \\
& =g(x) a e^{i\left(k_{y}^{0} y-\omega_{0} t\right)} \frac{1}{2 \pi} \frac{V_{g}\left(K_{1}\left(\omega_{0}\right)\right)}{\hat{g}\left(k_{x}^{0}\right)} \cos \left(\theta_{0}\right) .
\end{aligned}
$$

These results are a generalization of well known results in the literature. For the specific choice $g(x)=\exp \left(-\beta x^{2}\right)$ the last result is the same as the forcing derived by Wei e.a. [55]. If the function $g(x)$ is 
defined locally around $x=0$, i.e. $g(x)=\delta_{\text {Dirac }}(x)$ then the forcing equation is the same as the result of Kim e.a. [20]:

$$
S_{2}(x, y, t)=\delta_{\text {Dirac }}(x) a e^{i\left(k_{y}^{0} y-\omega_{0} t\right)} V_{g}\left(K_{1}\left(\omega_{0}\right)\right) \cos \left(\theta_{0}\right) .
$$

3. Generation of short crested wind waves. As a simple model of real water waves that have been generated by winds in coastal or oceanic waters, a multi-directional frequency-direction energy spectrum $E(\omega, \theta)$ is often adopted, see e.g. Holthuijsen [16]. The basis is a onesided energy spectrum $E_{1}(\omega)$ which is spread over the various directions through a normalized distribution function $D(\omega, \theta)$. For instance, $E_{1}(\omega)$ is a Jonswap, or Pierson-Moskowitz, spectrum, and $D(\theta)=\alpha \cos ^{2 s} \theta$ for $\theta \in(-\pi / 2, \pi / 2)$ and $D(\theta)=0$ else, where $\theta$ is the angle of the deviation from the averaged wave direction $\theta=0$; $s>0$ specifies the spreading, and $\alpha$ is a normalization factor. In order to prevent non-homogenity of the wave field which can be caused by multiple directions at one frequency, a one-parameter description of the wave field is then given by

$$
\eta(\mathbf{x}, t)=\Sigma_{n} \sqrt{E\left(\omega_{n}, \theta_{n}\right) \Delta \omega \Delta \theta} \cos \left(k_{x n} x+k_{y n} y-\omega_{n} t+\phi_{n}(\omega)\right)
$$

where $k_{x n}=k_{n} \cos \theta_{n}, k_{y n}=k_{n} \sin \theta_{n}, \phi_{n}(\omega)$ is a random phase, and $\Delta \omega \Delta \theta$ is determined by the bin-width of the spectrum division. Specified at the $y$-axis the wave is given by

$$
\eta(0, y, t)=\Sigma_{n} \sqrt{E\left(\omega_{n}, \theta_{n}\right) \Delta \omega \Delta \theta} \cos \left(y k_{n} \sin \theta_{n}-\omega_{n} t+\phi_{n}(\omega)\right)
$$

which specifies the influx signal along the $y$-axis to be used in the source function (3.13).

\subsection{Multi-directional propagating dispersive wave models}

In the first subsection we derive a source function for the second order dispersive wave equation with arbitary dispersion relation. In the second and 
third subsection we formulate the source for the Hamiltonian equation in $1 \mathrm{D}$ and $2 \mathrm{D}$ respectively. All wave models in this section describe waves that can propagate in any direction; particularly for the 1D case they describe waves travelling to the left and to the right.

\subsubsection{Second order dispersive wave model}

Using the excitation function for the (first order) forward propagating waves, it is possible to find the excitation function for the (second order) multi-directional dispersive wave equation.

Multi-directional waves can be obtained from influxing to the forward and to the backward separately, i.e by performing the two forcing equations:

$$
\begin{array}{r}
\partial_{t} \eta_{f}=-\mathcal{A}_{\mathbf{e}} \eta_{f}+S_{0}(\mathbf{x}, t), \quad \text { with } \quad \eta_{f}(0, y, t)=s_{0}(y, t) \\
\partial_{t} \eta_{b}=\mathcal{A}_{\mathbf{e}} \eta_{b}+S_{1}(\mathbf{x}, t), \quad \text { with } \quad \eta_{b}(0, y, t)=s_{1}(y, t),
\end{array}
$$

with $S_{0}(\mathbf{x}, t)$ and $S_{1}(\mathbf{x}, t)$ given by relation $(3.12)$. Then $\eta=\eta_{b}+\eta_{f}$ will be the solution for

$$
\begin{aligned}
\partial_{t} \eta & =\partial_{t}\left(\eta_{b}+\eta_{f}\right) \\
& =\left(\mathcal{A}_{\mathbf{e}} \eta_{b}-\mathcal{A}_{\mathbf{e}} \eta_{f}\right)+S_{0}(\mathbf{x}, t)+S_{1}(\mathbf{x}, t) .
\end{aligned}
$$

For the case $s_{0}(y, t)=s_{1}(y, t)=s(y, t)$, we get $\eta(0, y, t)=2 s(y, t)$. By taking the time derivative on both sides, we obtain the well known second order equation with its embedded source term:

$$
\partial_{t}^{2} \eta=-\mathcal{D} \eta+\partial_{t}\left[S_{0}(\mathbf{x}, t)+S_{1}(\mathbf{x}, t)\right] .
$$

Here the operator $\mathcal{D}=-\mathcal{A}_{\mathbf{e}}^{2}$ has as symbol $D$, i.e. using Fourier transformation, $D=\left(\Omega_{2}\left(k_{x}, k_{y}\right)\right)^{2}$.

For later reference, the solution of the equation (3.16) is given by

$$
\eta(x, y, t)=\sum_{j=0}^{1} \iint \frac{i \omega}{\omega^{2}-\Omega_{2}^{2}\left(k_{x}, k_{y}\right)} \bar{S}_{j}\left(k_{x}, k_{y}, \omega\right) e^{i\left(k_{x} x+k_{y} y-\omega t\right)} \mathbf{d k} d \omega
$$


Then

$$
\begin{aligned}
\eta(-x, y, t) & =\sum_{j=0}^{1} \iint \frac{i \omega}{\omega^{2}-\Omega_{2}^{2}\left(k_{x}, k_{y}\right)} \bar{S}_{j}\left(k_{x}, k_{y}, \omega\right) e^{i\left(-k_{x} x+k_{y} y-\omega t\right)} \mathbf{d k} d \omega \\
& =\sum_{j=0}^{1} \iint \frac{i \omega}{\omega^{2}-\Omega_{2}^{2}\left(k_{x}, k_{y}\right)} \bar{S}_{j}\left(-k_{x}, k_{y}, \omega\right) e^{i\left(k_{x} x+k_{y} y-\omega t\right)} \mathbf{d k} d \omega .
\end{aligned}
$$

Note that if $S_{0}=S_{1}$ then if $\bar{S}_{0}$ is an even function in $k_{x}$ we have symmetric propagating waves around the $y$-axis and if $\bar{S}_{0}$ is an odd function in $k_{x}$ we have a skew-symmetric influxing.

The same result also holds for the 1D case for which the result of the wave generation is that waves propagate (skew-)symmetrically to the left and to the right of the generation point.

\subsubsection{D Hamiltonian wave model}

The 1D second order dispersive equation $\partial_{t}^{2} \eta(x, t)=-\mathcal{D} \eta(x, t)$ can be written as the (Hamiltonian) system of two first order equations for the surface elevation $\eta(x, t)$ and the surface potential $\phi(x, t)$

$$
\begin{aligned}
\partial_{t} \eta & =\frac{\mathcal{D}}{g} \phi \\
\partial_{t} \phi & =-g \eta,
\end{aligned}
$$

with $\mathcal{D} \hat{=} \Omega_{1}^{2}(k)$. The first equation is the continuity equation and the second one is the momentum equation which is the linear Bernoulli equation. For the wave elevation $\eta$ and the wave potential function $\phi$ to be uniquely determined, two conditions are required for (3.18).

The embedded wave generation approach is the problem to determine $F_{1}$ and $F_{2}$ in the following system:

$$
\begin{aligned}
\partial_{t} \eta & =\frac{\mathcal{D}}{g} \phi+F_{1}(x, t) \\
\partial_{t} \phi & =-g \eta+F_{2}(x, t),
\end{aligned}
$$


so that at the generation position $x=0$ either $\eta$ or $\phi$ satisfies a given condition. The fact that only one condition is prescribed will make the source functions $F_{1}$ and $F_{2}$ in the equation (3.19) non-unique.

\section{Symmetric bi-directional elevation excitation}

Using the same method as in the forward propagating wave model, after Fourier transform equation (3.19) becomes:

$$
\left(\begin{array}{ll}
-i \omega & -\Omega_{1}^{2} / g \\
g & -i \omega
\end{array}\right)\left(\begin{array}{l}
\bar{\eta}(k, \omega) \\
\bar{\phi}(k, \omega)
\end{array}\right)=\left(\begin{array}{l}
\bar{F}_{1}(k, \omega) \\
\bar{F}_{2}(k, \omega)
\end{array}\right)
$$

from which the solution for $\bar{\eta}$ and $\bar{\phi}$ are given by:

$$
\left(\begin{array}{c}
\bar{\eta}(k, \omega) \\
\bar{\phi}(k, \omega)
\end{array}\right)=\frac{1}{\Omega_{1}^{2}-\omega^{2}}\left(\begin{array}{cc}
-i \omega & \Omega_{1}^{2} / g \\
-g & -i \omega
\end{array}\right)\left(\begin{array}{c}
\bar{F}_{1}(k, \omega) \\
\bar{F}_{2}(k, \omega)
\end{array}\right) .
$$

Suppose that the prescribed condition is a time trace of wave elevation at $x=0$, i.e. $\eta(0, t)=s(t)$, then

$$
\begin{aligned}
\check{s}(\omega) & =\int \bar{\eta}(k, \omega) d k \\
& =\int \frac{-i \omega \bar{F}_{1}(k, \omega)+\left(\Omega_{1}^{2} / g\right) \bar{F}_{2}(k, \omega)}{\Omega_{1}^{2}-\omega^{2}} d k \\
& =\int\left[\frac{\frac{\bar{F}_{1}\left(K_{1}(\nu), \omega\right)}{2}+\frac{i \nu \bar{F}_{2}\left(K_{1}(\nu), \omega\right)}{2 g}}{i V_{g}\left(K_{1}(\nu)\right)(\nu-\omega)}+\frac{\frac{\bar{F}_{1}\left(K_{1}(\nu), \omega\right)}{2}-\frac{i \nu \bar{F}_{2}\left(K_{1}(\nu), \omega\right)}{2 g}}{i V_{g}\left(K_{1}(\nu)\right)(-\nu-\omega)}\right] d \nu,
\end{aligned}
$$

with $K_{1}(\nu)$ the inverse of the dispersion relation function $\Omega_{1}(k)$. Using Cauchy's principle value theorem and the fact that $V_{g}$ is symmetric in $k$ 
while $K_{1}(\omega)$ is a skew symmetric function we obtain:

$$
\begin{aligned}
& \check{s}(\omega)=2 \pi\left[\begin{array}{l}
\frac{1}{V_{g}\left(K_{1}(\omega)\right)}\left(\frac{\bar{F}_{1}\left(K_{1}(\omega), \omega\right)}{2}+\frac{i \omega \bar{F}_{2}\left(K_{1}(\omega), \omega\right)}{2 g}\right)+ \\
\frac{1}{V_{g}\left(K_{1}(-\omega)\right)}\left(\frac{\bar{F}_{1}\left(K_{1}(-\omega), \omega\right)}{2}+\frac{i \omega \bar{F}_{2}\left(K_{1}(-\omega), \omega\right)}{2 g}\right)
\end{array}\right] \\
& =\frac{2 \pi}{V_{g}\left(K_{1}(\omega)\right)}\left[\begin{array}{l}
\left(\frac{\bar{F}_{1}\left(K_{1}(\omega), \omega\right)}{2}+\frac{i \omega \bar{F}_{2}\left(K_{1}(\omega), \omega\right)}{2 g}\right)+ \\
\left(\frac{\bar{F}_{1}\left(-K_{1}(\omega), \omega\right)}{2}+\frac{i \omega \bar{F}_{2}\left(-K_{1}(\omega), \omega\right)}{2 g}\right)
\end{array}\right] .
\end{aligned}
$$

We will choose $\bar{F}_{1}$ to be even in $k$ and $\bar{F}_{2}=0$. As a consequence we obtain a symmetric wave generation and with this setting we have:

$$
\check{s}(\omega)=\frac{2 \pi}{V_{g}\left(K_{1}(\omega)\right)} \bar{F}_{1}\left(K_{1}(\omega), \omega\right),
$$

or equivalently:

$$
\bar{F}_{1}\left(K_{1}(\omega), \omega\right)=\frac{1}{2 \pi} V_{g}\left(K_{1}(\omega)\right) \check{s}(\omega) .
$$

The source function is the same as the source function (3.7) in the 1D forward propagating wave model. But notice that for the same time signal at the generation position, the amplitude of the generated waves using Hamiltonian model will be half of the amplitude of the generated waves from forward propagating waves model. This is due to the fact that the Hamiltonian model with source (3.21) produces waves travelling to the forward and to the backward direction symmetrically. Or stated differently, the energy from the influxing will be distributed equally in both directions. This symmetry in influxing can be seen as follows.

The velocity at the generation point can be calculated as follows:

$$
\begin{aligned}
\partial_{x} \phi(0, t) & =\iint i k \bar{\phi}(k, \omega) e^{-i \omega t} d k d \omega \\
& =\iint i k\left(\frac{-g}{\Omega_{1}^{2}-\omega^{2}} \bar{F}_{1}(k, \omega)\right) e^{-i \omega t} d k d \omega .
\end{aligned}
$$


Since $\frac{\bar{F}_{1}}{\left(\Omega^{2}-\omega^{2}\right)}$ is an even function in $k$, the integrand is an odd function. Hence we obtain that for this elevation excitation the velocity at the generation point vanishes, i.e:

$$
\partial_{x} \phi(0, t)=0 .
$$

Further inspection shows $\partial_{x} \eta(0, t)=0$. This proves the symmetry of the waves to the right and to the left from the generation point.

\section{Uni-directional elevation influxing}

In this section we will restrict the forcing functions $F_{1}$ and $F_{2}$ in the Hamiltonian formulation (3.19) in such a way that the result of the influxing is that the waves travel only to the right; the elevation signal is given at the generation point $x=0$, i.e. $\eta(0, t)=s(t)$.

Wei e.a. in [55] use a source function which is spatially symmetric around $x=0$ for the bi-directional wave model. The result is a symmetric wave generation around $x=0$. In this section we combine a symmetric with a suitable skew-symmetric influxing so that the contributions at one side of the generation area will cancel.

According to (3.17), if $g(x)$ is a symmetric function in $x$ and the function $f(t)$ is chosen such that it satisfies relation (3.9) the solution of $\partial_{t}^{2} \eta=$ $-\mathcal{D} \eta+2 g(x) \partial_{t} f(t)$ will be symmetric in $x$ and can be written as

$$
\eta(x, t)=\iint \frac{2 i \omega}{\omega^{2}-\Omega_{1}^{2}(k)} \check{f}(\omega) \hat{g}(k) e^{i(k x-\omega t)} d k d \omega .
$$

The skew-symmetric function $\zeta=\partial_{x} \eta$ satisfies $\partial_{t}^{2} \zeta=-\mathcal{D} \zeta+2 \partial_{x} g(x) \partial_{t} f(t)$ and its solution is

$$
\zeta(x, t)=\iint \frac{2 i \omega}{\omega^{2}-\Omega_{1}^{2}(k)} \check{f}(\omega) i k \hat{g}(k) e^{i(k x-\omega t)} d k d \omega .
$$

Observe that equation (3.22) and equation (3.23) differ only in a factor of $i k$. Therefore if for $\zeta$ we give a temporal function $\mathcal{K} f(t)$ with $\mathcal{K}=1 / i k(\omega)$ instead of $f(t)$, i.e. $\partial_{t}^{2} \zeta=-\mathcal{D} \zeta+2 \partial_{x} g(x) \partial_{t} \mathcal{K} f(t)$, we will have $\zeta(x, t)=$ $\eta(x, t)$ for $x>0$ and $\zeta(x, t)=-\eta(x, t)$ for $x<0$. 
The uni-directional elevation influxing for the second order wave model can then be obtained by

$$
\partial_{t}^{2} \eta=-\mathcal{D} \eta+g(x) \partial_{t} f(t)+\partial_{x} g(x) \partial_{t} \mathcal{K} f(t)
$$

But since the operator $-\partial_{t} \mathcal{K}=C$ with $C$ is the phase velocity operator we can rewrite equation (3.24) as

$$
\partial_{t}^{2} \eta=-\mathcal{D} \eta+g(x) \partial_{t} f(t)-\partial_{x} g(x) C f(t) .
$$

For the Hamiltonian model the uni-directional elevation influxing can be obtained by eleminating $\phi$ from equation (3.19):

$$
\partial_{t}^{2} \eta+\mathcal{D} \eta=\frac{\mathcal{D}}{g} F_{2}+\partial_{t} F_{1}
$$

and equating equation (3.25) and (3.26), i.e.

$$
g(x) \partial_{t} f(t)-\partial_{x} g(x) C f(t)=\frac{\mathcal{D}}{g} F_{2}+\partial_{t} F_{1} .
$$

The sources $F_{1}$ and $F_{2}$ are then given as follows:

$$
\begin{aligned}
& F_{1}(x, t)=g(x) f(t)-\partial_{x} g(x) C \int f(t) d t \\
& F_{2}(x, t)=0 .
\end{aligned}
$$

\section{Uni-directional velocity influxing}

For $F_{1}(x, t)=0$, by eliminating wave elevation $\eta$ from the forced Hamiltonian formulation (3.19) we get

$$
\partial_{t}^{2} \phi+\mathcal{D} \phi=\partial_{t} F_{2} .
$$

This is the same as formulation for the uni-directional elevation influxing given in the previous section, and if the potential is given at the origin, i.e. $\phi(0, t)=\phi_{0}(t)$, the function $F_{2}$ is given as follows:

$$
F_{2}(x, t)=g(x) f(t)-\partial_{x} g(x) C \int f(t) d t
$$


In case the elevation $\eta_{0}(t)=\eta(0, t)$ is prescribed, we determine the corresponding potential signal from the Bernoulli equation $\partial_{t} \phi=-g \eta$, so $\phi_{0}(t)=-g \int \eta_{0}(t) d t$.

For the formulation in velocity variable $u=\partial_{x} \phi$, the corresponding equation is:

$$
\partial_{t} u=-g \partial_{x} \eta+\partial_{x} F_{2}
$$

where $F_{2}$ is determined by $(3.27)$ for prescribed velocity potential at the origin. In case the velocity $u(0, t)=\partial_{x} \phi(0, t)=u_{0}(t)$ is prescribed the source $F_{2}$ is given by (3.27) with the function $g(x)$ and $f(t)$ should satisfy the following equation:

$$
\check{f}(\omega)=\frac{1}{2 \pi i} \frac{V_{g}\left(K_{1}(\omega)\right)}{K_{1}(\omega) \hat{g}\left(K_{1}(\omega)\right)} \check{u}_{0}(\omega) .
$$

\subsubsection{D Hamiltonian wave model}

In this section, we will treat the 2D Hamiltonian wave generation model. The model is given by:

$$
\begin{aligned}
\partial_{t} \eta & =\frac{\mathcal{D}}{g} \phi+F_{1}(x, y, t) \\
\partial_{t} \phi & =-g \eta+F_{2}(x, y, t),
\end{aligned}
$$

with $\mathcal{D} \hat{=} \Omega_{2}^{2}\left(k_{x}, k_{y}\right)$. For later reference, the solution of equation (3.29) in Fourier space is given by:

$$
\left(\begin{array}{c}
\bar{\eta}\left(k_{x}, k_{y}, \omega\right) \\
\bar{\phi}\left(k_{x}, k_{y}, \omega\right)
\end{array}\right)=\frac{1}{\Omega_{2}^{2}-\omega^{2}}\left(\begin{array}{cc}
-i \omega & \Omega_{2}^{2} / g \\
-g & -i \omega
\end{array}\right)\left(\begin{array}{c}
\bar{F}_{1}\left(k_{x}, k_{y}, \omega\right) \\
\bar{F}_{2}\left(k_{x}, k_{y}, \omega\right)
\end{array}\right) .
$$

As we did for the 1D Hamiltonian case, we derive the bi-directional elevation influxing and the uni-directional (elevation or velocity) influxing. 


\section{Symmetric bi-directional elevation excitation}

Suppose that we excite waves along the $y$-axis, i.e: $\eta(0, y, t)=s(y, t)$ then

$$
\begin{aligned}
\check{s}\left(k_{y}, \omega\right) & =\int \bar{\eta}\left(k_{x}, k_{y}, \omega\right) d k_{x} \\
& =\int \frac{-i \omega \bar{F}_{1}\left(k_{x}, k_{y}, \omega\right)+\left(\Omega_{2}^{2} / g\right) \bar{F}_{2}\left(k_{x}, k_{y}, \omega\right)}{\Omega_{2}^{2}-\omega^{2}} d k_{x} .
\end{aligned}
$$

As in $1 \mathrm{D}$, to get symmetric generation, in the following we will choose $\bar{F}_{2}=0$ and $\bar{F}_{1}$ to be an even function in $k_{x}$ and hence we have:

$$
\check{s}\left(k_{y}, \omega\right)=\int \frac{-i \omega \bar{F}_{1}\left(k_{x}, k_{y}, \omega\right)}{\Omega_{2}^{2}-\omega^{2}} d k_{x} .
$$

Now we make a change of integration variable from $k_{x}$ to $\nu=\Omega_{2}\left(k_{x}, k_{y}\right)$, which is possible because of the monotony of $\Omega_{2}$ with respect to $k_{x}$ at fixed $k_{y}$, leading to $k_{x}=K_{x}\left(k_{y}, \nu\right)$. Writing $K\left(k_{y}, \nu\right)=\sqrt{K_{x}^{2}+k_{y}^{2}}$ and using $d \nu / d k_{x}=\operatorname{sign}\left(k_{x}\right) \partial \Omega_{1} / \partial k . \partial k / \partial k_{x}=V_{g}(k)\left|k_{x}\right| / k$ we have :

$$
\check{s}\left(k_{y}, \omega\right)=\int \frac{K\left(k_{y}, \nu\right)\left(-i \omega \bar{F}_{1}\left(K_{x}\left(k_{y}, \nu\right), k_{y}, \omega\right)\right)}{V_{g}\left(K\left(k_{y}, \nu\right)\right)\left|K_{x}\left(k_{y}, \nu\right)\right|\left(\nu^{2}-\omega^{2}\right)} d \nu .
$$

By Cauchy's integral theorem we obtain:

$$
\check{s}\left(k_{y}, \omega\right)=\frac{2 \pi}{V_{g}\left(K\left(k_{y}, \omega\right)\right)} \frac{K\left(k_{y}, \omega\right)}{\left|K_{x}\left(k_{y}, \omega\right)\right|} \bar{F}_{1}\left(K_{x}\left(k_{y}, \omega\right), k_{y}, \omega\right)
$$

or equivalently:

$$
\bar{F}_{1}\left(K_{x}\left(k_{y}, \omega\right), k_{y}, \omega\right)=\frac{1}{2 \pi} V_{g}\left(K\left(k_{y}, \omega\right)\right) \frac{\left|K_{x}\left(k_{y}, \omega\right)\right|}{K\left(k_{y}, \omega\right)} \check{s}\left(k_{y}, \omega\right) .
$$

Observe that equation (3.30) is the same as equation (3.12).

Equation $(3.30)$ can be rewritten as $\bar{F}_{1}\left(k_{x}, k_{y}, \omega\right)=\hat{g}\left(k_{x}\right) \check{f}\left(k_{y}, \omega\right)$. So for a given function $g(x)$, then

$$
\check{f}\left(k_{y}, \omega\right)=\frac{1}{2 \pi} \frac{V_{g}\left(K\left(k_{y}, \omega\right)\right)}{\hat{g}\left(K_{x}\left(k_{y}, \omega\right)\right)} \frac{\left|K_{x}\left(k_{y}, \omega\right)\right|}{K\left(k_{y}, \omega\right)} \check{s}\left(k_{y}, \omega\right) .
$$


The velocity at the generation line can be calculated as follows:

$$
\begin{aligned}
\partial_{x} \phi(x=0, y, t) & =\iint i k_{x} \bar{\phi}\left(k_{x}, k_{y}, \omega\right) e^{i\left(k_{y} y-\omega t\right)} \mathbf{d k} d \omega \\
& =\iint i k_{x}\left(\frac{-g}{\Omega_{2}^{2}-\omega^{2}} \bar{F}_{1}\left(k_{x}, k_{y}, \omega\right)\right) e^{i\left(k_{y} y-\omega t\right)} \mathbf{d k} d \omega,
\end{aligned}
$$

but since $\frac{\bar{F}_{1}}{\left(\Omega_{2}^{2}-\omega^{2}\right)}$ is an even function in $k_{x}$, the integrand is an odd function in $k_{x}$, hence for the case of the symmetric bi-directional elevation excitation it holds that the velocity vanishes along the generation line, i.e:

$$
\partial_{x} \phi(0, y, t)=0 .
$$

\section{Uni-directional elevation influxing}

We will determine the forcing function $F_{1}$ and $F_{2}$ in the 2D Hamiltonian formulation so that for a given signal of wave elevation $s(y, t)$ along the $y$-axis the result of the influxing is forward propagating waves only.

Similar to the 1D case, the uni-directional elevation influxing in the 2D case can be obtained by

$$
\partial_{t}^{2} \eta=-\mathcal{D} \eta+g(x) \partial_{t} f(y, t)+\partial_{x} g(x) \partial_{t} \mathcal{K}_{x} f(y, t)
$$

where $g(x)$ and $f(y, t)$ satisfy relation (3.31) and the operator $\mathcal{K}_{x}$ is defined by $1 / k_{x}$ in Fourier space.

On the other hand, from the forced Hamiltonian formulation we get

$$
\partial_{t}^{2} \eta+\mathcal{D} \eta=\frac{\mathcal{D}}{g} F_{2}+\partial_{t} F_{1}
$$

Equating equation (3.32) and (3.33) we have

$$
g(x) \partial_{t} f(y, t)+\partial_{x} g(x) \partial_{t} \mathcal{K}_{x} f(y, t)=\frac{\mathcal{D}}{g} F_{2}+\partial_{t} F_{1} .
$$

The sources $F_{1}$ and $F_{2}$ are then given as follows:

$$
\begin{aligned}
& F_{1}(x, y, t)=g(x) f(y, t)+\partial_{x} g(x) \mathcal{K}_{x} f(y, t) \\
& F_{2}(x, y, t)=0 .
\end{aligned}
$$




\section{Uni-directional velocity influxing}

For $F_{1}(x, y, t)=0$ and by eliminating wave elevation $\eta$ from the forced Hamiltonian formulation (3.29) we get

$$
\partial_{t}^{2} \phi+\mathcal{D} \phi=\partial_{t} F_{2}
$$

If the potential is given at the $y$-axis, i.e. $\phi(0, y, t)=\phi_{0}(y, t)$, the function $F_{2}$ can be found in the same way as for the uni-directional elevation influxing, and is given as:

$$
F_{2}(x, y, t)=g(x) f(y, t)+\partial_{x} g(x) \mathcal{K}_{x} f(y, t) .
$$

In case the elevation signal $s_{\eta}(y, t)$ at the $y$-axis is given, the corresponding potential signal can be found from the evolution equation $\partial_{t} \phi=-g \eta$, so

$$
\bar{s}_{\phi}\left(k_{y}, \omega\right)=-i g \bar{s}_{\eta}\left(k_{y}, \omega\right) / \omega .
$$

For the formulation in velocity variable $\mathbf{u}=(u, v)=\nabla \phi$, the corresponding equation is:

$$
\partial_{t} \mathbf{u}=-\nabla(g \eta)+\nabla F_{2},
$$

where $F_{2}$ is determined by (3.34) for prescribed velocity potential at the $y$-axis.

\subsection{Numerical simulations}

In this section, we show the performance of the influx methods by simulations with implementations in two numerical codes. One code is a spectral implementation of the equations with exact dispersion relation; the other code is a Finite Element implementation of the Optimized Variational Boussinesq Model (OVBM), see [23].

The spectral implementation can deal easily with the exact dispersion relations and its approximations, such as in [48],[50].

For working in Matlab, one has to observe a multiplicative factor $\alpha=$ $2 \pi / d x$ if we start with the expression in Fourier-space and apply IFFT. Indeed, starting with $\hat{g}(k)$ we get 


$$
g(x)=\operatorname{IFFT}(\hat{g})=\frac{1}{N} \Sigma \hat{g}_{k} e^{i k x}
$$

while

$$
\gamma(x)=\int \hat{\gamma}(k) e^{i k x} d k \approx \Sigma \hat{\gamma}(k) e^{i k x} \Delta k
$$

so

$$
\frac{1}{N} \hat{g}_{k}=\hat{\gamma}(k) \Delta k \rightarrow \hat{g}_{k}=\hat{\gamma}(k) \Delta k \cdot N=\hat{\gamma}(k) \frac{2 \pi}{d x} .
$$

For the $2 \mathrm{D}$ case, the multiplication factor is $(2 \pi)^{2} /(d x . d y)$.

To show results of $1 \mathrm{D}$ simulations in the spectral simulations, we use the AB-model that has exact linear dispersion and accurate up to and including the second order terms; see [46] for details. For the 2D case, we use a spectral implementation of the AB2-model which is derived in Chapter 2.

Besides these spectral models with exact dispersion, we show results with a finite element implementation of the Variational Boussinesq Method (see [3],[22], [23]). The simulations of Variational Boussinesq Model that are shown here are performed by Dr. Aditya.

\subsubsection{D Spectral implementation: Nonlinear wave focusing}

In this section, we show the usefulness of the excitation models for nonlinear cases where we compare the nonlinear $\mathrm{AB}$ simulations with real measurement from MARIN hydrodynamic laboratory (Marine Research Institute Netherlands). The MARIN measurement to be compared is the case 109001. The signal is given at $x=0$ on a long tank basin with depth of $1[m]$. After 1 minute experiment, the evolution of the generated wave is measured at positions $x=19.2[\mathrm{~m}]$ and $x=20.8[\mathrm{~m}]$.

The simulation is done by embedded point generation in the nonlinear AB-equation. The influx signal consists of short waves which have slower speed followed by long waves which have faster speed. The broad spectrum, and the strong focusing effect (with more then threefold amplitude amplification compared to the maximal influx amplitudes), makes this a suitable test for the influx performance and particularly the nonlinear behavior. 
The plot of the measurement signal and the modified signal that is used in the source term for the AB simulation are depicted at the upper left side of figure (3.3). We notice that the modified signal has a higher amplitude and higher spectrum because of the multiplication with the group velocity as in expression (3.8).
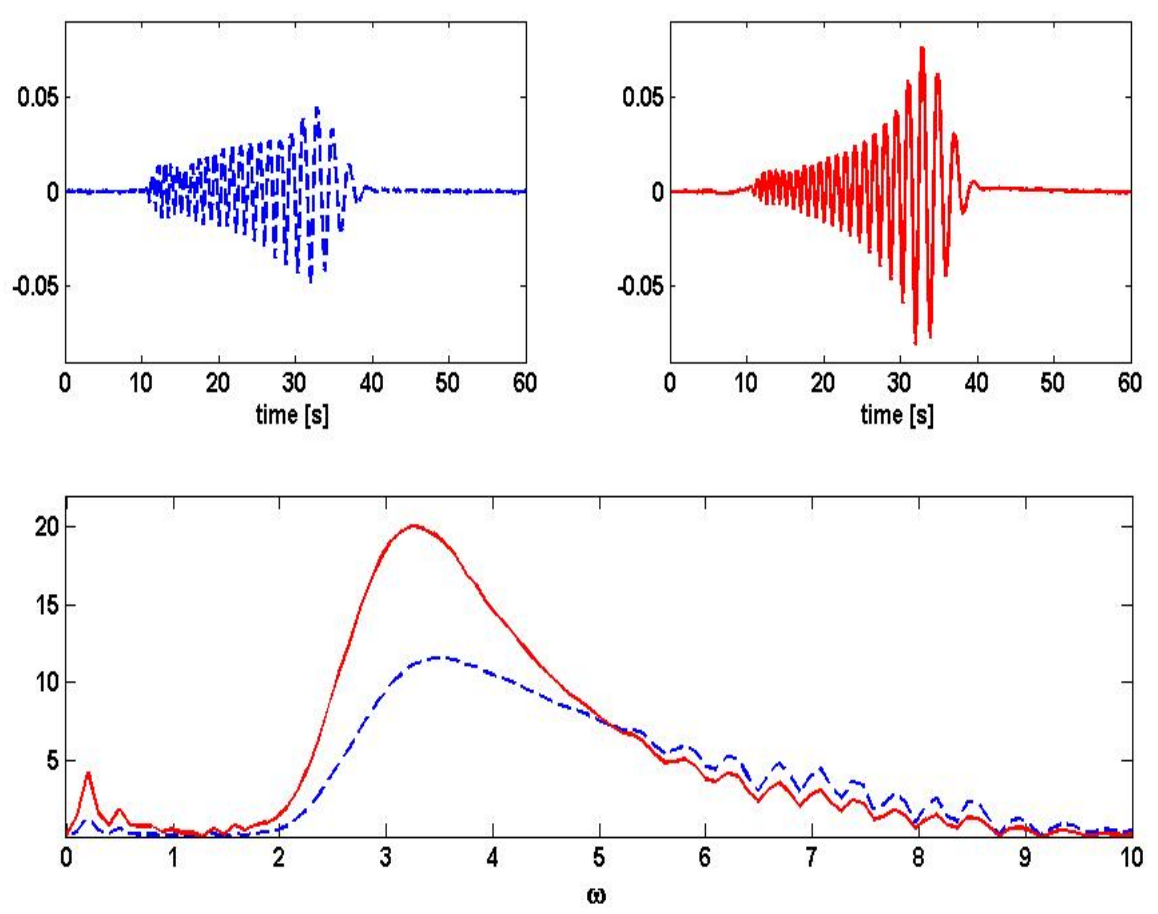

Figure 3.3: Plot of the influx signals and their amplitude spectrum. Shown on the left of the first row is the laboratory signal at the excitation position while the right side is its related modified signal to be used in the source function of point generation of the AB simulation. The second row is the (amplitude) spectrum for the laboratory signal (dashed line) and the modified signal (solid line).

The results of the numerical simulation are presented in figure (3.4). Here we plot part of the time signal from the AB simulation at the indi- 

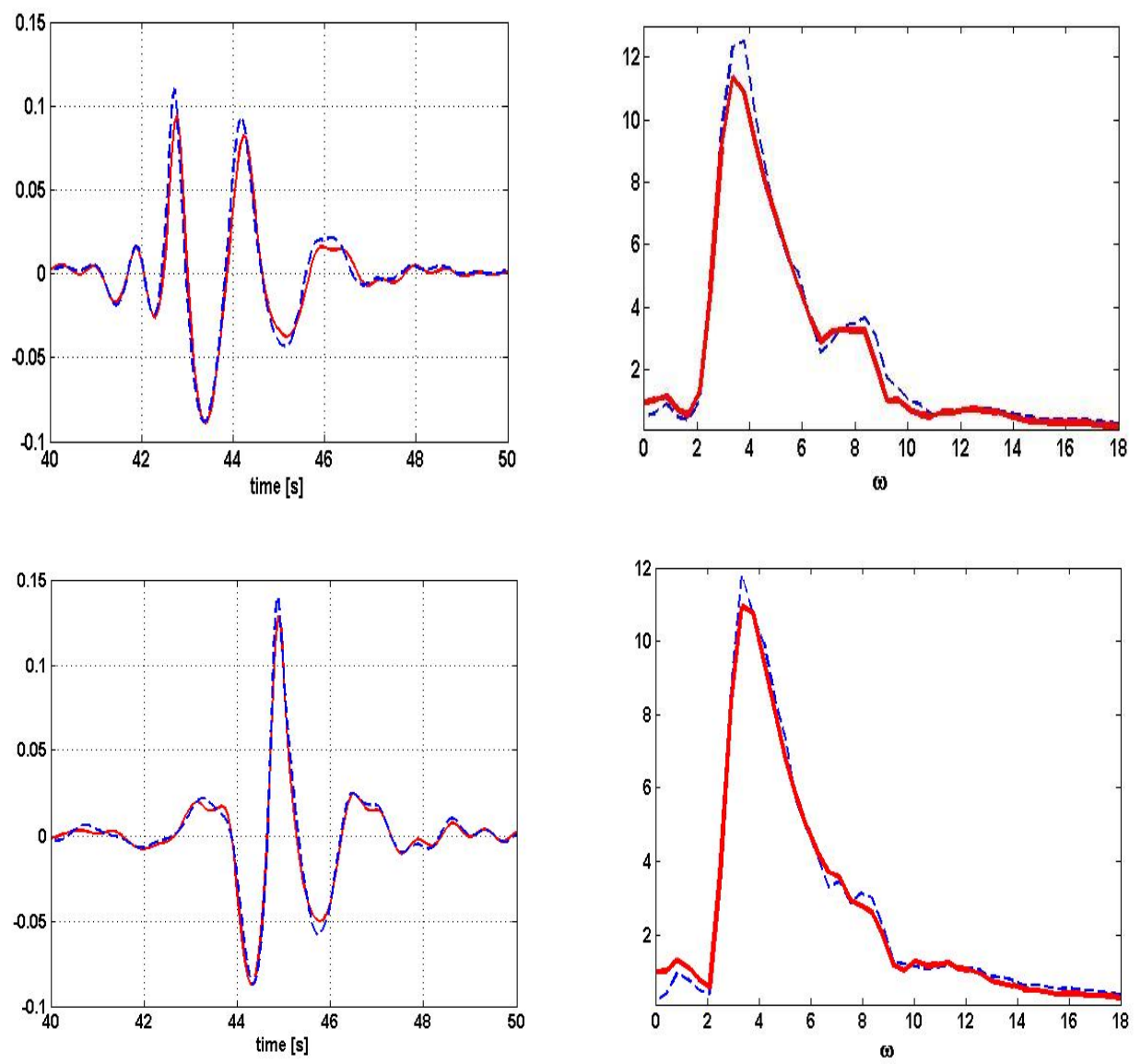

Figure 3.4: Part of the time signal in the first column and the amplitude spectrum in the second column as $A B$ simulation (solid curve) and measurement (dashed curve). The first row is the result of measurement and simulation at position $x=19.2[\mathrm{~m}]$ and the second row is at position $x=20.8[\mathrm{~m}]$.

cated measurement position together with its related measurement signal. The spectrum of the signal from the measurement and simulation are also presented. 
Figure (3.4) shows clearly the phenomenon of wave focusing where longer waves chase shorter waves until eventually catching up and constructively interfere at the focusing point. As illustrated in figure (3.4) we see that the excitation model with the nonlinear AB-equation is able to capture the deformations of the influxing waves rather accurately with only a slight difference in the time signal. The spectra show that the long waves are reasonably well simulated, just as well as the high frequency waves.

\subsubsection{Finite element implementation: Uni-directional influx- ing}

In this section, several 1D simulations are performed by using a finite element code of the VBM to illustrate the uni-directional influxing if either the wave elevation or the wave velocity is prescribed at the generation position. Simulations are done for $60[\mathrm{~s}]$ and on $0.5[\mathrm{~m}]$ water depth. The computational domain is from $x=-40[m]$ until $x=40[m]$ and the wave generation is done at the origin.

The influxed signal for the elevation influxing is chosen to be a bipolar given by $\eta_{0}(t)=0.2(t-30) e^{-(t-30)^{2}}$ and the initial signal for the velocity influxing is given by $\check{\phi}_{0}(\omega)=\frac{g}{i \omega} \check{\eta}_{0}(\omega)$.

For each case of elevation and velocity influxing, three separate simulations are performed using a symmetric, a skew-symmetric and a combination of a symmetric and a skew-symmetric (in space) source function. For the spatial dependence in the source of equation (3.25) and (3.28), $g(x) \hat{=} \frac{1}{2 \pi} V_{g}(k)$ is taken. Figure (3.5) depicts the plots of the simulations result for both the elevation (on the left panel) and the velocity influxing (on the right panel). The graphs show wave profile snapshots after $40[s]$ simulation. It is visible from the graph that the generated waves depend on the type of the spatial profile in the source. Apart from the elevation around the neighborhood of the origin, both elevation and velocity generation give the same result. 

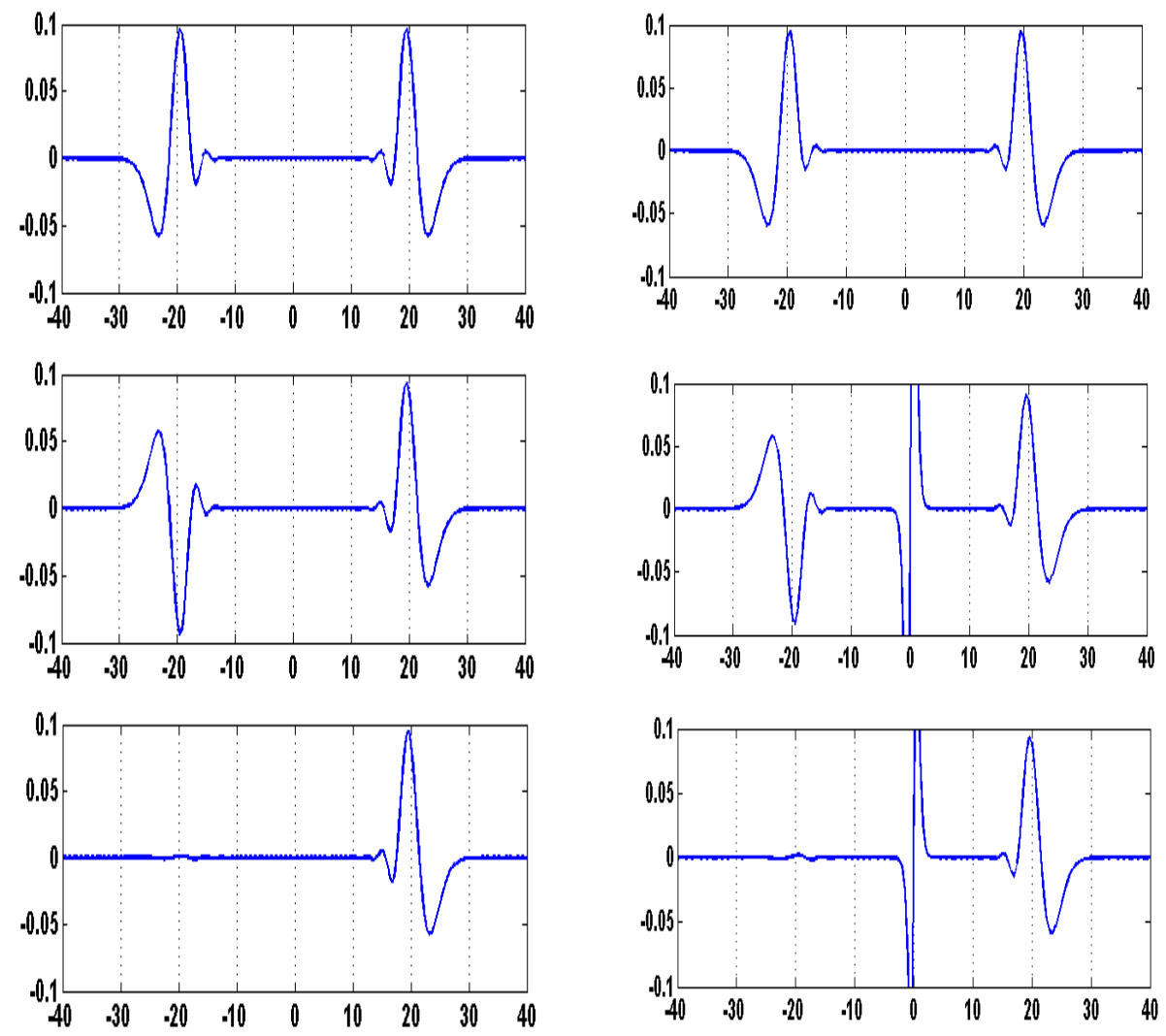

Figure 3.5: Snapshots of the wave profile at $t=40[s]$. The horizontal and the vertical axis are the space and the wave elevation respectively and are measured in $[m]$. The first column shows results of wave generation with elevation influxing while the second column shows results with velocity influxing. In successive order, from the first to the last row, the result of the generation is shown if a symmetric, a skew-symmetric and a uni-directional source is used. 


\subsection{Conclusion}

This chapter started with the question how to incorporate the effect of a flap motion in the laboratory into the dynamic equations of water waves. It is essential to make such an excitation model accurately as slight errors will lead after propagation over large distances to large errors.

For dispersive wave equations, we showed that we can incorporate the flap motion in an embedded way into the dynamic equation by adding a force term to the equation. The force function is not uniquely determined; there are many choices that can be made for this force function. Two limiting cases are the area extended and the point (or line) excitation. For the area extended excitation the original time signal to be influxed is kept to be the same, while for the point excitation (or line excitation in 2D) the time signal needs to be modified so that the influx is done only at a single point (or along a line in 2D). In all cases, the physical variable that plays a role is the wave group velocity $\left(V_{g}\right)$.

For multi-directional waves where the governing equation is given by the Hamiltonian system or the second order equation, it is possible to perform influxing not only symmetrically to the forward and to the backward but also influxing only in the forward direction by combining symmetric and skew symmetric influxing. In the last case, we can achieve forward elevation and forward velocity influxing. These uni-directional influxing for Boussinesq type models have the advantage to reduce the computational domain, which will improve efficiency of numerical simulations.

Two test cases to show the robustness and the accuracy of the excitation models have been performed. Using the nonlinear AB-equation for testing the case of focusing waves gives less than $3 \%$ error in comparison with the measurement data from the laboratory (in a sense of the highest wave elevation at the focussing position). The result of the oblique wave interaction using the AB2-equation and influxing from the $x$ - and $y$-axis simultaneously will be shown in the next chapter. 


\section{Chapter 4}

\section{Experiment and simulation of oblique wave interaction}

\subsection{Introduction}

An extensive study about wave group evolutions has been done by Westhuis in 1999. It is motivated by the appearance of large deformations of waves that are of relevance for hydrodynamic laboratories. He performed a series of (1D) experiment to investigate the effect of nonlinearity on the deformation of wave groups. A bichromatic signal, $s(t)=a \cos \left(\omega_{1} t\right)+a \cos \left(\omega_{2} t\right)$, was used for the stroke of the wave flap in the laboratory. The complete setup of his experiment was reported in [56] and [57]. He found that for small initial amplitudes $a$ the wave elevation differs only very little from linear theory and for larger amplitudes the deformation of wave group is largely influenced by the difference of the frequency $\omega_{1}$ and $\omega_{2}$ at the flap signal. Figure (4.1) and figure (4.2) show two measurement results from his experiments.

Using the same wave parameters of the experiments, we performed several experiments in 2010 at MARIN hydrodynamic laboratory. But instead of performing a one dimensional experiment, where wave generation is only 

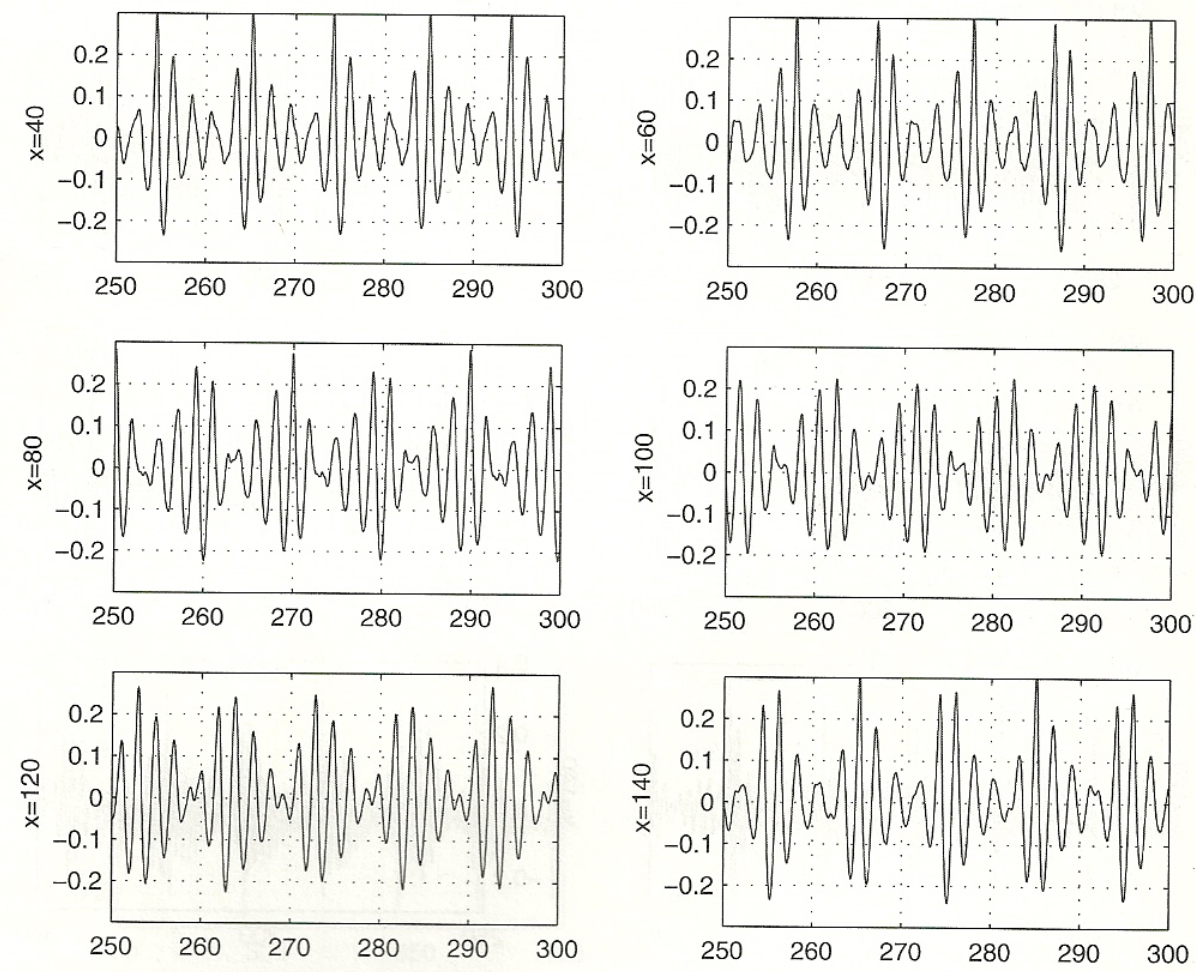

Figure 4.1: Experiment results of Westhuis: bichromatic wave evolution at different positions in the tank. The vertical axis is the wave elevation in $[\mathrm{m}]$ and the horizontal axis is the time in $[s] . \quad\left(T_{1}=1.8[\mathrm{~s}], T_{2}=2.2[\mathrm{~s}], h=5[\mathrm{~m}], a=\right.$ $0.1[m]$, MARIN test no.6371)

done from one side of the water tank with all flaps pushing in the same way, we performed two dimensional experiments with influx from the $x$-axis and from the $y$-axis simultaneously; as influx signals we choose harmonic waves with different periods. This chapter is devoted to the results of these experiments and their numerical simulations.

The aim of the experiment is first to produce the behavior of bichromatic wave groups as found earlier by letting two harmonic (plane) waves 

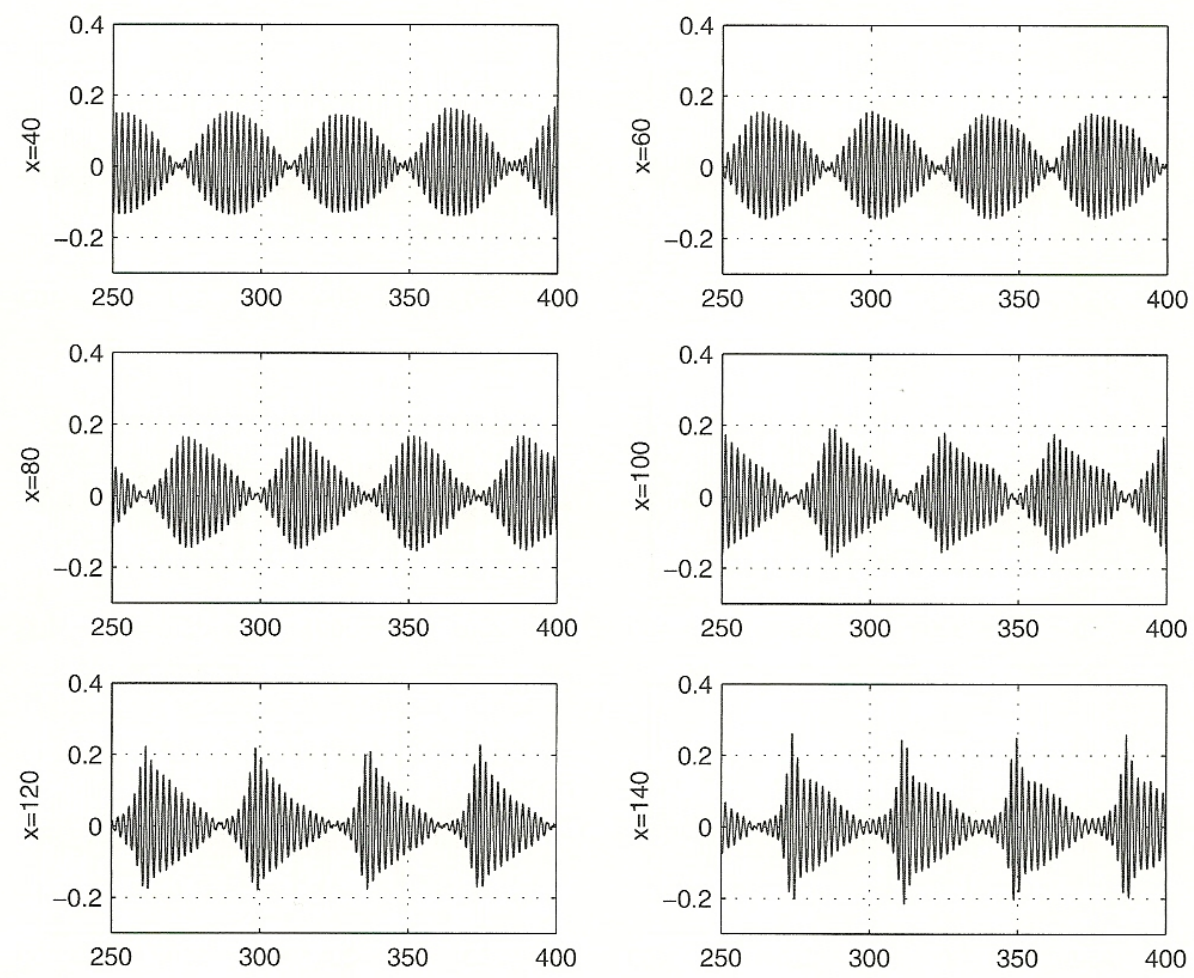

Figure 4.2: Experiment results of Westhuis: bichromatic wave evolution at different positions in the tank. The vertical axis is the wave elevation in $[\mathrm{m}]$ and the horizontal axis is the time in $[s] . \quad\left(T_{1}=1.95[s], T_{2}=2.05[s], h=5[\mathrm{~m}], a=\right.$ $0.07[m]$, MARIN test no.6387)

propagating from two different directions collide with one another. Secondly, the experimental results can be used to validate the applicability and the performance of the AB2-model and the two dimensional influxing techniques that have been discussed in detail in previous chapters.

The next section describes the laboratory experimental set-up and the layout of the measurements. After this description we provide the numerical framework that is used for the simulation. The comparison between the 
experiment and the simulation are provided in section 4, and conclusions in section 5 .

\subsection{Experimental setting}

\subsubsection{Physical outlook of water basin}

As part of the STW-project (TWI.7216), the experiments were conducted at MARIN hydrodynamics laboratory in Wageningen, The Netherlands, in the seakeeping and manoeuvering basin (SMB). This basin has dimension of $170[\mathrm{~m}]$ long, $40[\mathrm{~m}]$ wide and $5[\mathrm{~m}]$ deep. It is designed to perform seakeeping tests (the response of a ship to waves). In this basin, wind can also be generated during the test by using an adjustable $10[\mathrm{~m}]$ wide platform with electrical fans. See the website of MARIN [52] for more information about the facilities. Waves are generated by forcing the flap-type wavemaker on two sides of the basin and are absorbed by an artificial beach at the opposing sides. There are 63 and 268 flaps on the short and on the long side of the basin respectively. Each flap has a width of $0.6[\mathrm{~m}]$ and its hinge lies 1.6 $[\mathrm{m}]$ below the still water level. A prescribed signal is given to the flap motion control software for the stroke of the flaps. Figure (4.3) depicts the seakeeping and manoeuvering basin of MARIN laboratory (Source: [52]).

Several electronic wave probes are used during the experiments to measure the (time signal of the) wave elevation. In all probes the sampling period is $0.01[s]$. These electronic wave probes are attached to a carriage and they are installed in the centerline of the tank $(y=19.42[\mathrm{~m}])$. Three probes are installed around $x=40[m], x=80[m], x=120[m]$. With this positioning, we expect to catch sufficient information on deformation of the waves. Figure (4.4) illustrates the arrangement of the wave probes that were used during the experiments. The black dots in the picture denote the electronic probes attached to the carriage. 


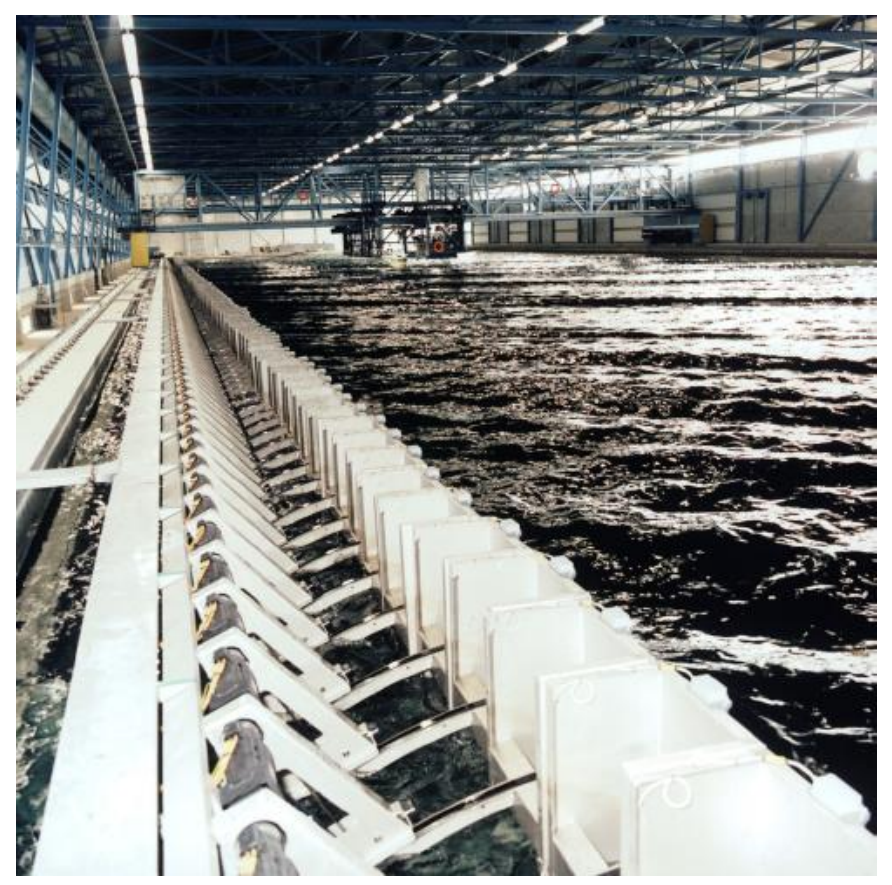

Figure 4.3: Photograph of the MARIN's seakeeping and manoeuvering basin, see $[52]$.

\subsubsection{Designed test cases}

As explained in the introductory section the original aim of the experiment is to investigate the nonlinear behavior of two dimensional bichromatic wave group by influxing two harmonic waves from two sides of the basin. To this end, we start from the fact that a (one dimensional) bichromatic wave group is a result from a linear combination of two regular waves with two different wave lengths (or wave periods) and equal amplitude, or stated in formula we write it as: 

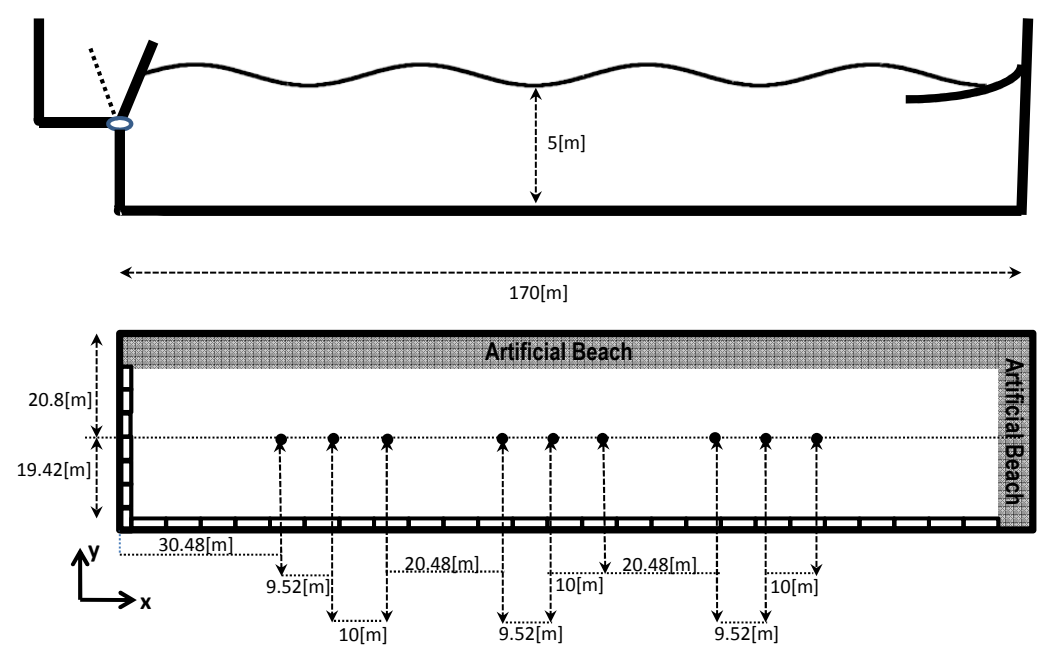

Figure 4.4: Sketch of the water basin and the arrangement of the probes. Shown on the lower graph, the wave makers are located at the west and south side of the basin and the artificial beaches are located at the east and north side of the basin.

$$
\begin{aligned}
\eta(x, t) & =a \cos \left(k_{1} x-\omega_{1} t\right)+a \cos \left(k_{2} x-\omega_{2} t\right) \\
& =2 a \cos (\Delta k x-\Delta \omega t) \cos (\bar{k} x-\bar{\omega} t)
\end{aligned}
$$

where $\omega_{1}, \omega_{2}$ are wave frequencies related to the wave numbers $k_{1}, k_{2}$, and $a$ is the wave amplitude. Here $\Delta k=\left(k_{1}-k_{2}\right) / 2, \Delta \omega=\left(\omega_{1}-\omega_{2}\right) / 2$, $\bar{k}=\left(k_{1}+k_{2}\right) / 2$ and $\bar{\omega}=\left(\omega_{1}+\omega_{2}\right) / 2$. We can interpret the bichromatic wave as a carrier wave $(\bar{k}, \bar{\omega})$ modulated by an envelope wave $(\Delta k, \Delta \omega)$.

In the two dimensional setting, the linear interaction of two oblique waves where one wave propagates in the direction of $\theta_{1}$ (with respect to the positive $x$-axis) and another one travels in the direction $\theta_{2}$ is given by

$$
\eta(x, y, t)=a_{1} \cos \left(k_{1 x} x+k_{1 y} y-\omega_{1} t\right)+a_{2} \cos \left(k_{2 x} x+k_{2 y} y-\omega_{2} t\right)
$$


with $a_{1}, a_{2}$ are amplitudes and $\omega_{1}, \omega_{2}$ are frequencies. Here $\left(k_{1 x}, k_{1 y}\right)=$ $K\left(\omega_{1}\right)\left(\cos \theta_{1}, \sin \theta_{1}\right)$ and $\left(k_{2 x}, k_{2 y}\right)=K\left(\omega_{2}\right)\left(\cos \theta_{2}, \sin \theta_{2}\right)$. Supposing that $\theta_{1}=0$ and $a_{1}=a_{2}$ we have

$$
\eta(x, y, t)=a \cos \left(K\left(\omega_{1}\right) x-\omega_{1} t\right)+a \cos \left(k_{2 x} x+k_{2 y} y-\omega_{2} t\right) .
$$

At $y=0[m]$, the wave profile is then given by

$$
\begin{aligned}
\eta(x, y=0, t) & =a \cos \left(K\left(\omega_{1}\right) x-\omega_{1} t\right)+a \cos \left(k_{2 x} x-\omega_{2} t\right) \\
& =2 a \cos (\Delta \kappa x-\Delta \omega t) \cos (\bar{\kappa} x-\bar{\omega} t),
\end{aligned}
$$

with $\Delta \kappa=\left(K\left(\omega_{1}\right)-k_{2 x}\right) / 2$ and $\bar{\kappa}=\left(K\left(\omega_{1}\right)+k_{2 x}\right) / 2$. From here we see that unless $\theta_{2}=\pi / 2$ the bichromatic profile (4.5) will be different from the one written in (4.2).

From an experimental point of view, the linear wave profile (4.4) can be obtained by influxing from the short and the long side of the basin simultaneously. For our experiments we set the influx at the short side of the tank to be fixed from $y=10.2[\mathrm{~m}]$ until $y=26.4[\mathrm{~m}]$ while at the long side the influx is fixed from $x=11.2[\mathrm{~m}]$ until $x=150[\mathrm{~m}]$. As influx signal at the short side of the basin $(x=0)$ we prescribe

$$
s_{y}(t)=a \cos \left(\omega_{1} t\right)
$$

and at the long side of the basin $(y=0)$ we prescribe

$$
s_{x}(x, t)=a \cos \left(k_{2 x} x-\omega_{2} t-\phi\right)
$$

with the phase $\phi=19.42 K\left(\omega_{2}\right) \sin \left(\theta_{2}\right)$ and $\theta_{2}=\pi / 6$.

By using signal (4.6) and (4.7) for the stroke of the flaps, we obtain a plane wave (result of influxing from the $y$-axis) and an oblique wave that travel $30^{0}$ from the positive $x$-axis (result of influxing from the $x$-axis). After these waves collide, a wave profile as (4.5) is obtained at $y=19.42[\mathrm{~m}]$.

There are 12 different cases of experiments that have been done in MARIN during the period of 25 - 27 March 2010; 4 cases are influxing along the short side of the basin only, 4 other cases are influxing along 
the long side of the basin only and the remaining cases are influxing along the short and the long side of the basin simultaneously. For each of these cases there are 3 pairs of measurements; the first one is the measurement at $(30.48,19.42),(40,19.42),(50,19.42)$, the second one is the measurement at position $(70.48,19.42),(80,19.42),(90,19.42)$ and the last one is the measurement at $(110.48,19.42),(120,19.42),(130,19.42)$.

Table (4.1) shows the parameters of all tests that were executed. In this table, $a$ is the wave amplitude and $T$ is the wave period. Indexes 1 and 2 at the parameters are related to the direction of influxing : 1 indicates the influxing along the short side of the tank and 2 indicates the influxing along the long side. Notice that for each case (and condition) there are 3 associated test numbers; the first, second and third test number refer to measurement at position where the center of the carriage is at $(40,19.42),(80,19.42)$ and $(120,19.42)$ respectively. The time interval during which each measurement is done is $385[s]$.

\begin{tabular}{|c|c|c|c|}
\hline Case & Condition & Test No. & Parameters \\
\hline $1 \mathrm{a}$ & short side only & $\begin{array}{l}120004 \\
120005 \\
120006\end{array}$ & $a_{1}=0.1[m], T_{1}=1.8[s]$ \\
\hline $1 \mathrm{~b}$ & long side only & $\begin{array}{l}121002 \\
121003 \\
121004\end{array}$ & $a_{2}=0.1[m], T_{2}=2.2[s]$ \\
\hline $1 \mathrm{c}$ & short \& long side & $\begin{array}{l}122002 \\
122003 \\
122004\end{array}$ & $\begin{array}{l}a_{1}=0.1[m], T_{1}=1.8[s] \\
a_{2}=0.1[m], T_{2}=2.2[s]\end{array}$ \\
\hline $2 \mathrm{a}$ & short side only & $\begin{array}{l}123001 \\
123005 \\
123006\end{array}$ & $a_{1}=0.09[\mathrm{~m}], T_{1}=1.9[\mathrm{~s}]$ \\
\hline $2 \mathrm{~b}$ & long side only & $\begin{array}{l}124001 \\
124002 \\
124003\end{array}$ & $a_{2}=0.09[\mathrm{~m}], T_{2}=2.1[\mathrm{~s}]$ \\
\hline
\end{tabular}




\begin{tabular}{|c|c|c|c|}
\hline Case & Condition & Test No. & Parameters \\
\hline $2 \mathrm{c}$ & short \& long side & $\begin{array}{l}125001 \\
125002 \\
125003\end{array}$ & $\begin{array}{l}a_{1}=0.09[m], T_{1}=1.9[s] \\
a_{2}=0.09[m], T_{2}=2.1[s]\end{array}$ \\
\hline $3 \mathrm{a}$ & short side only & $\begin{array}{l}126001 \\
126002 \\
126003\end{array}$ & $a_{1}=0.09[\mathrm{~m}], T_{1}=1.925[\mathrm{~s}]$ \\
\hline $3 \mathrm{~b}$ & long side only & $\begin{array}{l}127001 \\
127002 \\
127003\end{array}$ & $a_{2}=0.09[\mathrm{~m}], T_{2}=2.075[\mathrm{~s}]$ \\
\hline $3 \mathrm{c}$ & short \& long side & $\begin{array}{l}128001 \\
128002 \\
128003\end{array}$ & $\begin{array}{l}a_{1}=0.09[\mathrm{~m}], T_{1}=1.925[\mathrm{~s}] \\
a_{2}=0.09[\mathrm{~m}], T_{2}=2.075[\mathrm{~s}]\end{array}$ \\
\hline $4 \mathrm{a}$ & short side only & $\begin{array}{l}129001 \\
129002 \\
129003\end{array}$ & $a_{1}=0.07[m], T_{1}=1.95[s]$ \\
\hline $4 \mathrm{~b}$ & long side only & $\begin{array}{l}130001 \\
130002 \\
130003\end{array}$ & $a_{2}=0.07[m], T_{2}=2.05[s]$ \\
\hline $4 \mathrm{c}$ & short \& long side & $\begin{array}{l}131001 \\
131002 \\
131003\end{array}$ & $\begin{array}{l}a_{1}=0.07[\mathrm{~m}], T_{1}=1.95[\mathrm{~s}] \\
a_{2}=0.07[\mathrm{~m}], T_{2}=2.05[\mathrm{~s}]\end{array}$ \\
\hline
\end{tabular}

Table 4.1: Overview of MARIN test cases

Figures $(4.5)-(4.8)$ show some of the result of the experiments: part of the time traces of the surface elevation at different positions for test cases with influxing from both sides of the basin.

In each of these figures we notice that the result of two plane wave interaction is a bichromatic wave for which the shape of the wave envelope appears to be deformed at the different positions in the basin. Figure (4.6) and figure (4.7) show that for the same amplitude the deformation of the wave envelope depends on the period difference of the two influxing signals; 
the smaller the difference the stronger the deformation of the wave group is.

From figure (4.5) - (4.8) we also see the deformation of the wave amplitude during the wave propagation; for instance in figure (4.5) the crest and the trough of the influx signal are $0.2[\mathrm{~m}]$ and $-0.2[\mathrm{~m}]$ respectively but their values at $x=80[\mathrm{~m}]$ from the measurement are $0.24[\mathrm{~m}]$ for the crest and $-0.18[\mathrm{~m}]$ for the trough.

The deformation of the wave envelope and the deviation of the wave amplitude is the effect of the nonlinearity which cannot be captured by the linear theory.

We also observe that the results from these two-dimensional experiments are different from the ones which are conducted by Westhuis and reported in [57]. For instance, in the case of strongly nonlinear bichromatic wave where the difference of two wave periods is very small we observe that the zero crossing in between two wave envelopes does not really occur in the two dimensional experiment, see figure (4.2) and figure (4.8). This might be due to the fact that two amplitudes used in the steering signal for the experiment are not exactly the same. Suppose that the signal at the short side of the tank has amplitude $a$ and amplitude $(a+\epsilon)$ at the long side then in the lowest order, the wave elevation at $y=19.42[\mathrm{~m}]$ is given by

$$
\begin{aligned}
\eta(x, y=19.42, t) & =a \cos \left(K\left(\omega_{1}\right) x-\omega_{1} t\right)+(a+\epsilon) \cos \left(k_{2 x} x-\omega_{2} t\right) \\
& =2 a \cos (\Delta \kappa x-\Delta \omega t) \cos (\bar{\kappa} x-\bar{\omega} t)+\epsilon \cos \left(k_{2 x} x-\omega_{2} t\right) .
\end{aligned}
$$

For $\epsilon=0$ we obtain a bichromatic wave with zero crossing in between two wave envelope, but for a nonzero $\epsilon$, the term $\epsilon \cos \left(k_{2 x} x-\omega_{2} t\right)$ will give an out of phase contribution depending on the position where the measurements are made. The higher order (nonlinearity) then makes the amplitude from this perturbation $(\epsilon)$ larger.

\subsection{Mathematical modeling and simulation}

Numerical simulations have been performed using the nonlinear AB2-equation [42] with an embedded source term described in Chapter 3 of this thesis. 

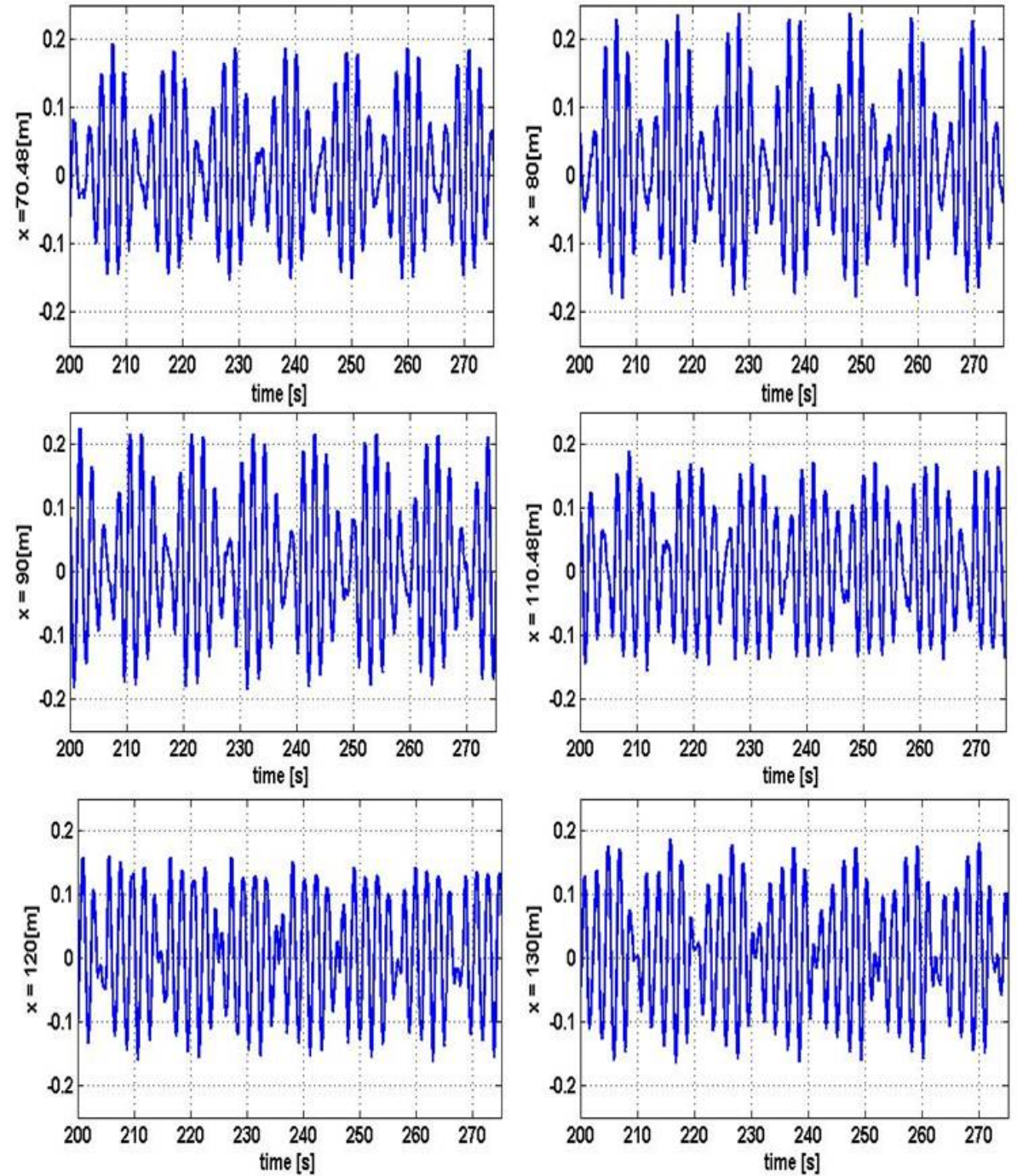

Figure 4.5: Plots of the time trace of measured wave elevation of two harmonic wave interaction at different positions in water basin $\left(T_{1}=1.8[s], T_{2}=2.2[s], a_{1}=\right.$ $a_{2}=0.1[m], h=5[m]$, MARIN test no.122002,122003 and 122004) 

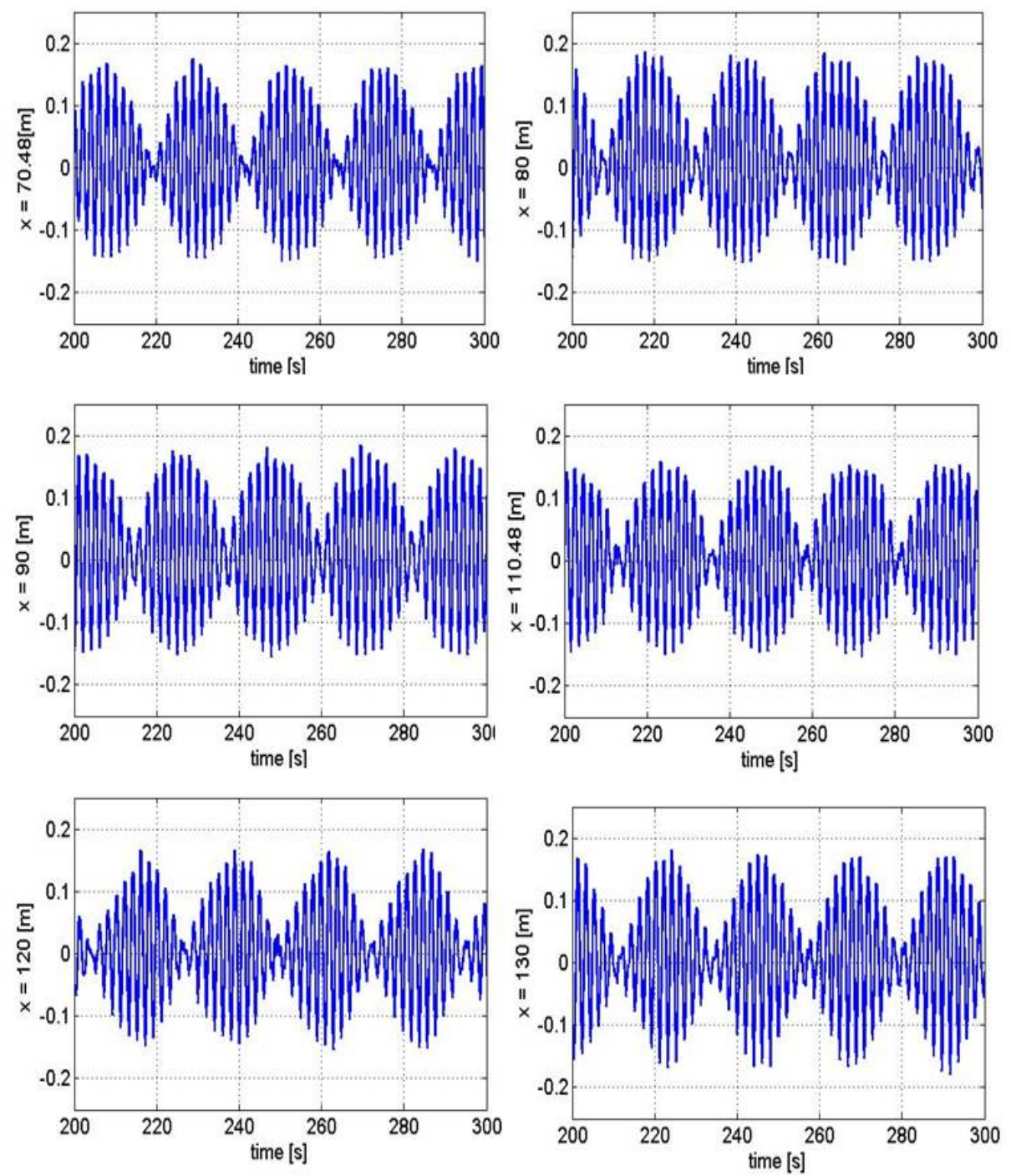

Figure 4.6: Plots of the time trace of measured wave elevation of two harmonic wave interaction at different positions in water basin $\left(T_{1}=1.9[s], T_{2}=2.1[s], a_{1}=\right.$ $a_{2}=0.09[m], h=5[m]$, MARIN test no.125001,125002 and 125003) 

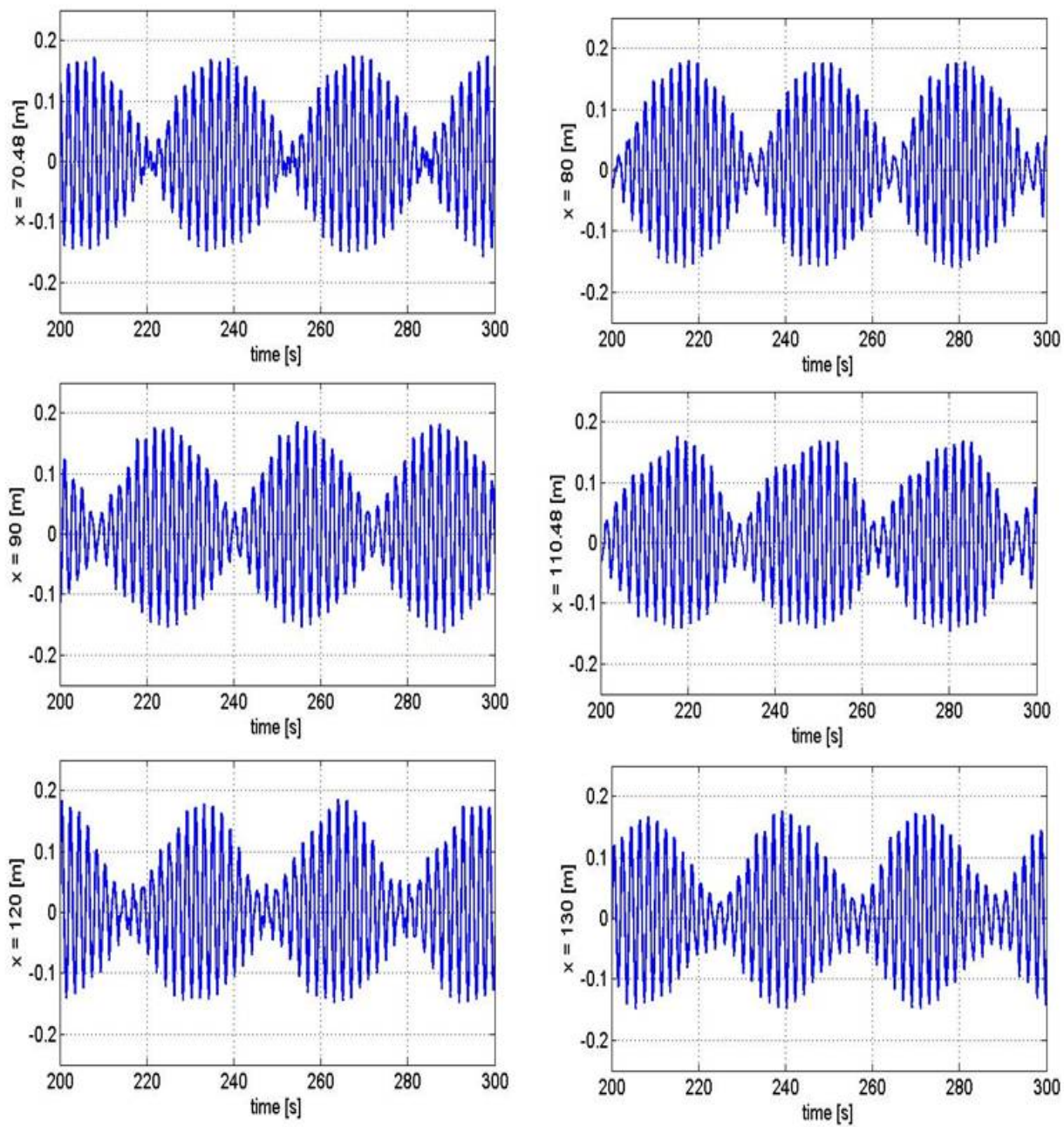

Figure 4.7: Plots of the time trace of measured wave elevation of two harmonic wave interaction at different positions in water basin $\left(T_{1}=1.925[\mathrm{~s}], T_{2}=\right.$ $2.075[s], a_{1}=a_{2}=0.09[m], h=5[m]$, MARIN test no.128001,128002 and 128003$)$ 

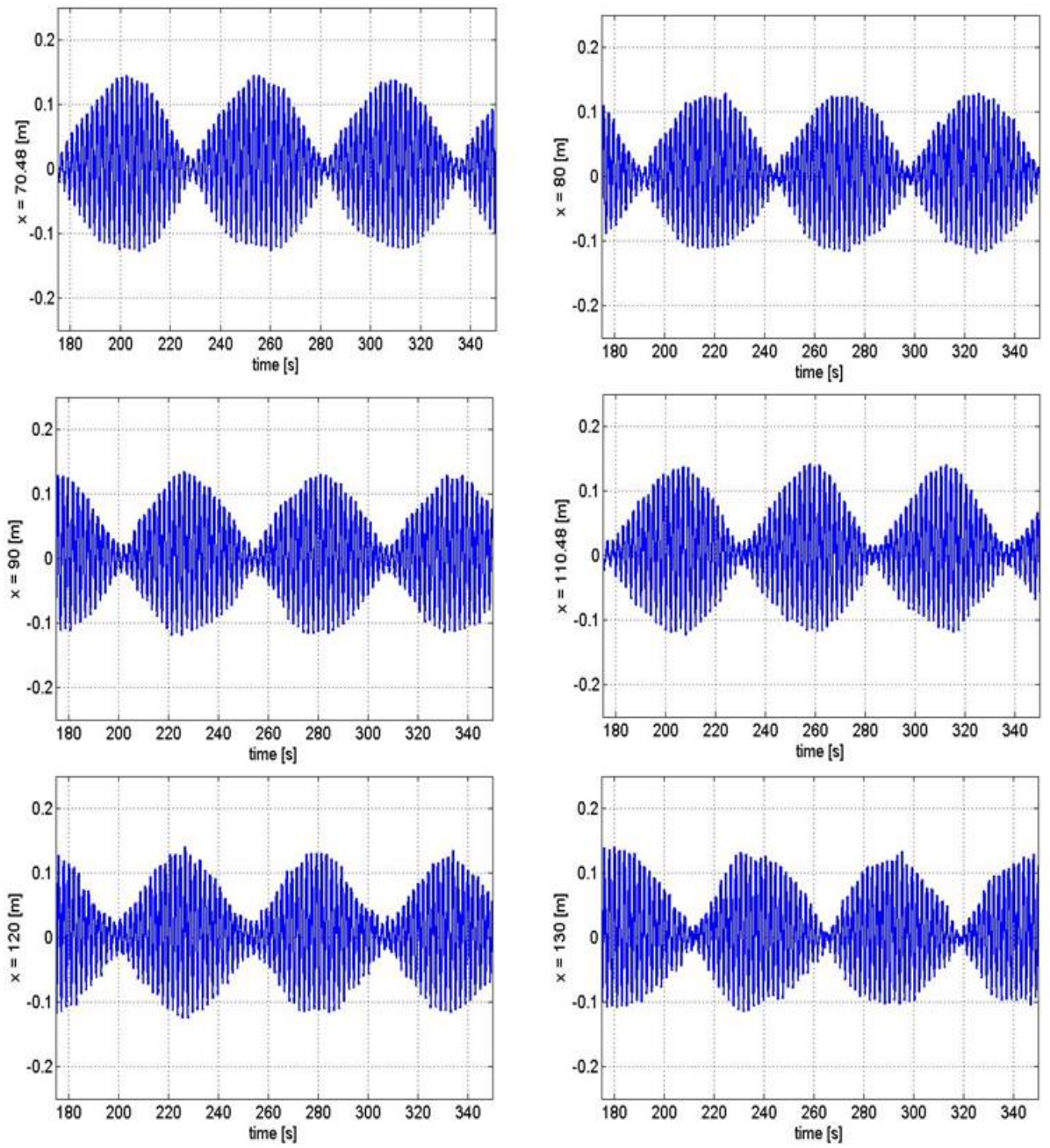

Figure 4.8: Plots of the time trace of measured wave elevation of two harmonic wave interaction at different positions in water basin $\left(T_{1}=1.95[s], T_{2}=\right.$ $2.05[s], a_{1}=a_{2}=0.07[m], h=5[m]$, MARIN test no.131001,131002 and 131003) 
The model is given as follows:

$$
\partial_{t} \eta=-\sqrt{g} A_{2}\left[\begin{array}{c}
\eta-\frac{1}{4}\left(A_{2} \eta\right)^{2}+\frac{1}{2} A_{2}\left(\eta A_{2} \eta\right) \\
+\frac{1}{4}\left(B_{2} \eta\right)^{2}+\frac{1}{2} B_{2}\left(\eta B_{2} \eta\right) \\
+\frac{1}{4}\left(\gamma_{2} \eta\right)^{2}+\frac{1}{2} \gamma_{2}\left(\eta \gamma_{2} \eta\right)
\end{array}\right]+S(x, y, t)-\alpha d(x, y) \eta
$$

The source term $S(x, y, t)$ is the sum of the two sources, i.e $S(x, y, t)=$ $S_{x}(x, y, t)+S_{y}(x, y, t)$ where $S_{x}(x, y, t)$ is the source along the long side of the basin (x-axis) and $S_{y}(x, y, t)$ is the source along the short side of the basin ( $y$-axis). Their expression are given explicitly by equation (3.15) of Chapter 3, i.e :

$$
\begin{gathered}
S_{x}(x, y, t)=\delta_{\text {Dirac }}(y) a_{2} e^{i\left(k_{2 x} x-\omega_{2} t\right)} V_{g}\left(K\left(\omega_{2}\right)\right) \sin \left(\theta_{0}\right) \\
S_{y}(x, y, t)=\delta_{\text {Dirac }}(x) a_{1} e^{-i \omega_{1} t} V_{g}\left(K\left(\omega_{1}\right)\right)
\end{gathered}
$$

with $a_{1}$ and $a_{2}$ are the wave amplitudes, $\omega_{1}$ and $\omega_{2}$ are the wave frequencies related to the wave period $T_{1}$ and $T_{2}$. Here $K(\omega)$ denotes the wavenumber related to the given frequency $\omega$ and $k_{2 x}=K\left(\omega_{2}\right) \cos \left(\theta_{0}\right)$ with $\theta_{0}=\pi / 6$.

The term $d(x, y) \eta$ is acting as an absorbing (or sponge layer) boundary condition which will dissipate the energy of the incoming wave. This type of boundary condition will force the wave elevation $\eta$ to decay near the end of the boundary. The larger the positive value of the damping coefficient $\alpha$ the faster the decaying. The function $d(x, y)$ defines the damping area and is given by a smoothened characteristic function which has value one in the damping zone and vanishes inside the domain of interest. We notice that the exponential decay of the solution $\eta$ in the damping area is of the order of $e^{-\alpha T_{l}}$ where $T_{l}$ is the travel time of the wave in the damping area. For instance, to obtain an exponential decay up to $10^{-3} \approx e^{-7}$ of the longest waves which have speed $c_{0}=\sqrt{g h}$, the width of the damping area should be at least $L_{d}=7 c_{0} / \alpha$.

We use a pseudo-spectral method for the implementation of the model. So instead of solving equation (4.8) in the physical space we transform it 
to the Fourier space and it becomes:

$$
\partial_{t} \hat{\eta}=-\sqrt{g} \hat{A_{2}} \cdot\left[\begin{array}{c}
\left.\hat{\eta}-\frac{1}{4} \widehat{\left(A_{2} \eta\right)^{2}}+\frac{1}{2} \widehat{A_{2}\left(\eta A_{2}\right.} \eta\right) \\
+\frac{1}{4} \widehat{\left(B_{2} \eta\right)^{2}}+\frac{1}{2} \widehat{\left.B_{2} \widehat{\left(\eta B_{2}\right.} \eta\right)} \\
+\frac{1}{4} \widehat{\left(\gamma_{2} \eta\right)^{2}}+\frac{1}{2} \widehat{\gamma_{2}\left(\widehat{\eta \gamma_{2} \eta}\right)}
\end{array}\right]+\widehat{S}(x, y, t)-\alpha \widehat{d(x, y) \eta}
$$

with the use of a hat-notation for the (2D) spatial Fourier transform. The nonlinear terms like $\widehat{\left(A_{2} \eta\right)^{2}}$ can be computed by using a convolution operation but it would be costly to do so. Therefore we use the following well known pseudo-spectral procedure instead:

1. For each time step $t$ we have $\hat{\eta}$, the Fourier transform of the surface elevation.

2. Multiply the operator $\hat{A}_{2}$ with $\hat{\eta}$ and hence we have $\widehat{A_{2} \eta}=\hat{A}_{2} \cdot \hat{\eta}$.

3. Apply the inverse Fourier transform to $\hat{A}_{2} \cdot \hat{\eta}$ and we obtain $A_{2} \eta$. Then we square it and take the Fourier transform to get the value of $\widehat{\left(A_{2} \eta\right)^{2}}$.

The procedure done for $\widehat{\left(A_{2} \eta\right)^{2}}$ can also be applied for the rest of the terms in the equation. All the Fourier transforms and its inverse are performed with the Fast Fourier Transform $(F F T)$ and the Inverse Fast Fourier Transform $(I F F T)$ in MATLAB.

The time stepping is done by an ODE-solver in MATLAB. For each time step we perform an anti-aliasing where we dump waves with wave vector $\mathbf{k}=\left(k_{x}, k_{y}\right)$ if $|\mathbf{k}| \geq\left(K_{\max } / 6\right)$; here $K_{\max }$ is related to the shortest wave in the simulation. The following parameters are chosen for the numerical simulation:

- $5[\mathrm{~m}]$ water depth and $350[\mathrm{~s}]$ simulation time.

- Domain of simulation is $[-15,185] \mathrm{x}[-15,60]$.

- Absorbing beach is applied at $x \notin[-5,165]$ and $y \notin[-5,40]$. 
- Individual wave makers for the simulation are positioned in the intervals $x \in[11,150]$ and $y \in[10,27]$; each wave maker has width $0.6[m]$ as in the laboratory.

As a consequence, instead of source (4.9) and source (4.10), the following sources are used:

$$
\begin{aligned}
S_{x}(x, y, t)= & \sum_{j=1}^{N_{x}} 1_{\left[x_{j}, x_{j+1}\right)}(x) e^{i k_{2 x} x_{j}} \delta_{\text {Dirac }}(y) a_{2} e^{-i \omega_{2} t} V_{g}\left(K\left(\omega_{2}\right)\right) \sin \left(\theta_{0}\right) \\
& S_{y}(x, y, t)=1_{[10,27]}(y) \delta_{\text {Dirac }}(x) a_{1} e^{-i \omega_{1} t} V_{g}\left(K\left(\omega_{1}\right)\right),
\end{aligned}
$$

with $N_{x}$ the number of flaps used for influxing from the $x$-axis, $x_{1}=11$ and $x_{j+1}=x_{j}+0.6$ for $j=1,2, \ldots, N_{x}$. Here $1_{[a, b)}(x)$ is a function with value one for $x \in[a, b)$ and zero otherwise.

In figure (4.9), the physical layout of the numerical implementation is shown. The direction of the wave propagation on the short side of the domain is to the right while on the long side of the domain the direction is $30^{0}$ with respect to the positive $x$-axis. Notice that we construct the damping area on the West and the South side of the numerical domain to be shorter than the damping area on the East and the North side. This is possible since the governing equation is the AB2-equation which is a forward propagating wave equation.

\subsection{Comparison between MARIN experiment and AB2-simulation}

In this section, we present results of simulations with the AB2-equation and we compare the results against the data from MARIN experiment. The simulation and the comparison are done both for the linear and the nonlinear AB2-equation. The linear simulation is performed by neglecting the higher order (quadratic) terms of the equation (4.11) while keeping the source and the damping to be the same. Here we present the comparison for the MARIN test case number 122002-122004 and 128001-128003. 

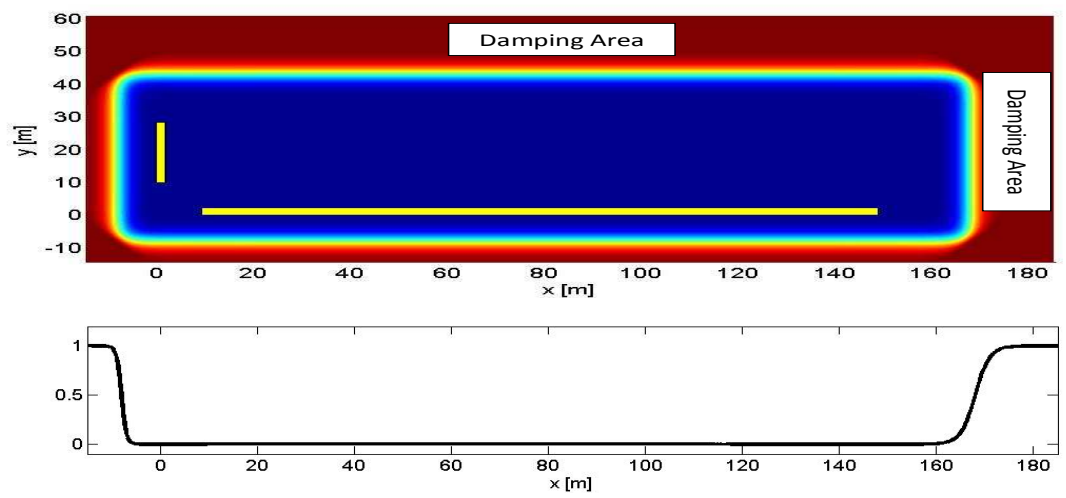

Figure 4.9: Shown on the upper graph is the layout for the numerical simulation of the wave generation using the AB2-equation. The lower graph is an illustration of the characteristic function for the damping area: the function $d(x, y)$ at a fixed $y$.

For each test case with parameters mentioned in section 4.3, on a computer with Pentium i7 processor every 5 minutes of simulation time takes about 9 minutes of computational time for the linear AB2-simulation and 40 minutes for the nonlinear AB2-simulation.

In the one dimensional case, we could use one (point) measurement data from the laboratory as influx signal for the simulation. Yet this situation is not possible with the two dimensional setting. The influx signal that we use for the AB2-simulation of oblique wave interaction is from the linear theory where the wave elevation at position $(x, y)=\left(x_{0}, y_{0}\right)$ can be written as:

$$
\eta_{0}(t)=\eta\left(x_{0}, y_{0}, t\right)=a_{1} \cos \left(\omega_{1} t-\phi_{1}\right)+a_{2} \cos \left(\omega_{2} t-\phi_{2}\right) .
$$

This function is then fitted with the measurement signal at $(80,19.42)$ by using curve fitting algorithm (fittype) in MATLAB. The fittype uses the least square method and the result produces the optimal values of parameters $a_{1}, a_{2}, \omega_{1}, \omega_{2}, \phi_{1}$ and $\phi_{2}$ that give the minimum error between the curve $\eta_{0}$ and the measurement signal at $(80,19.42)$. Then the parameters with index 1 are used for influxing along the $y$-axis while parameters with index 2 are used for influxing along the $x$-axis. In the following the values of these parameters for each test case are given. 


\section{MARIN test case number 122002-122004}

For the simulation we prescribe the influx signal at the short side of the domain to be a harmonic wave with amplitude $0.0855[\mathrm{~m}]$ and period $1.8[s]$ while at the long side we prescribe a harmonic wave with amplitude $0.1145[\mathrm{~m}]$ and period $2.174[\mathrm{~s}]$. To the signal at the short and long side of the domain we also add a phase $\phi_{1}=4.729$ and $\phi_{2}=6.543$ respectively. These parameters are obtained from the fitting procedure as explained above. The amplitudes for the simulation deviate $15 \%$ from the prescribed amplitudes in table (4.1) with lower amplitude at the short side and higher amplitude at the long side. We also observe that the wave period for the simulation at the long side differs $1 \%$ from the prescribed period.

Figure (4.10) and figure (4.11) are plots from the linear and the nonlinear AB2-simulation respectively. The time signals of the wave elevation are plotted at the positions $x=80,90,110.48,130[\mathrm{~m}]$ at $y=19.42[\mathrm{~m}]$. The vertical axis in these time signal plots is the elevation in meters and the horizontal axis is the time in seconds. In these figures, we also show a density plot which is the surface elevation seen from above after $350[s]$ simulation time. The wave profile (as a function of $x$ ) of the linear and the nonlinear AB2-equation at $y=19.42[\mathrm{~m}]$ after $350[\mathrm{~s}]$ are plotted together and shown in figure (4.12).

From figure (4.10) and figure (4.11), we see that the wave generation model with both the linear and the nonlinear AB2-equation captures rather accurately the deformation of the waves except for the small waves in between wave groups and a slight amplitude difference in the wave groups. It is noticeable from these figures that the nonlinearity of the AB2-equation makes an improvement at the crest and at the trough of the waves.

In table (4.2) we provide qualitative information about the comparison with the measurement. In that table we present the correlation and the variance-quotient between the simulation and the measurement; these are defined by

$$
\operatorname{Corr}\left(s_{1}, s_{2}\right)=\frac{<s_{1}(t), s_{2}(t)>}{\left|s_{1}(t)\right| \cdot\left|s_{2}(t)\right|} \text { and } V Q\left(s_{1}, s_{2}\right)=\frac{\left|s_{1}(t)\right|^{2}}{\left|s_{2}(t)\right|^{2}} .
$$



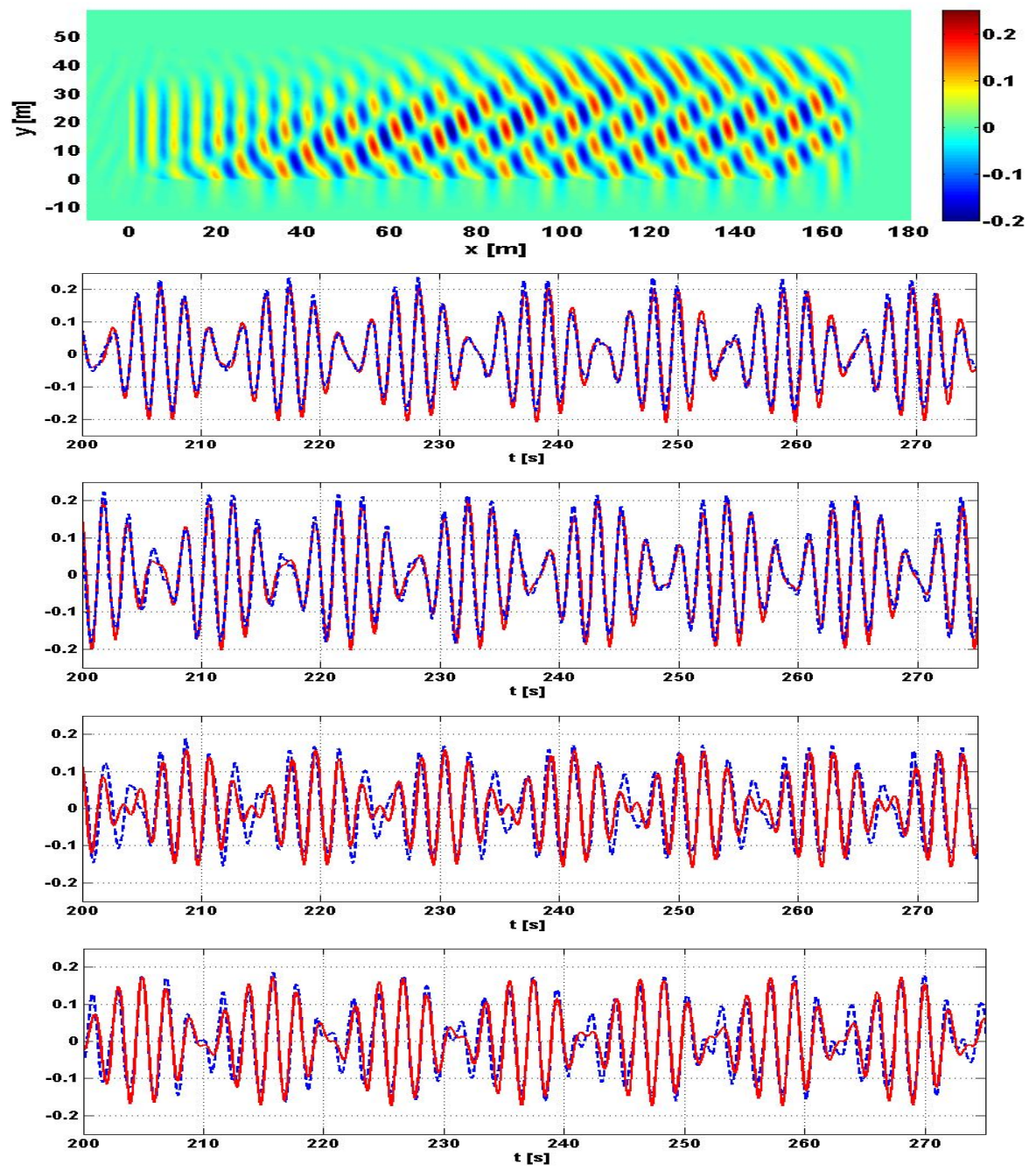

Figure 4.10: MARIN experiments and linear AB2-simulation: MARIN test no. 122002-122004. The first row is density plot after $t=350[s]$. From row 2 until row 5 successively are plots of time traces of the linear AB2-simulation (solid red) and the MARIN measurement (dashed-blue) at $y=19.42[\mathrm{~m}]$ and $x=80,90,110.48,130[m]$. 

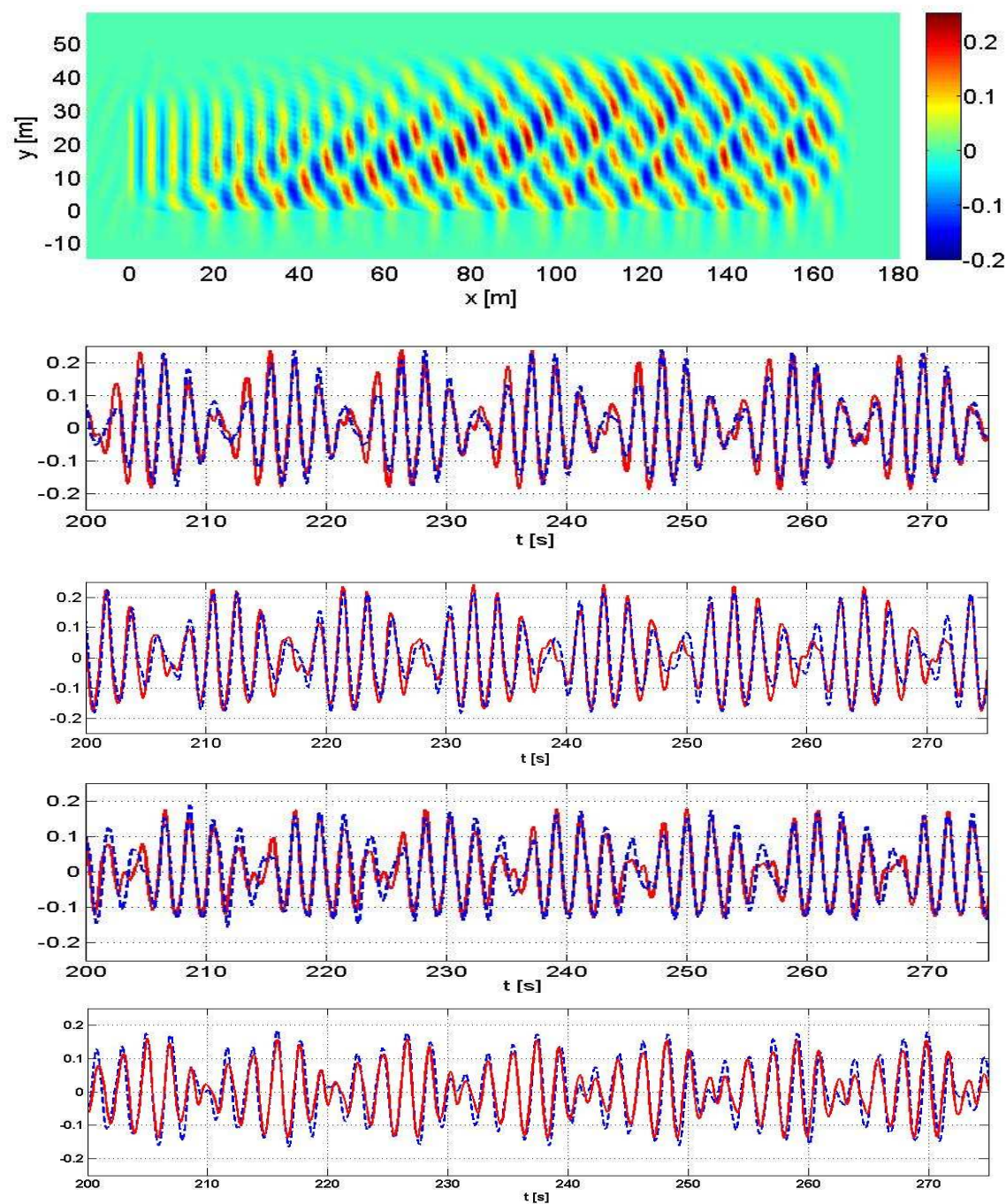

Figure 4.11: MARIN experiments and nonlinear AB2-simulation as in figure (4.10): MARIN test no. 122002-122004. 

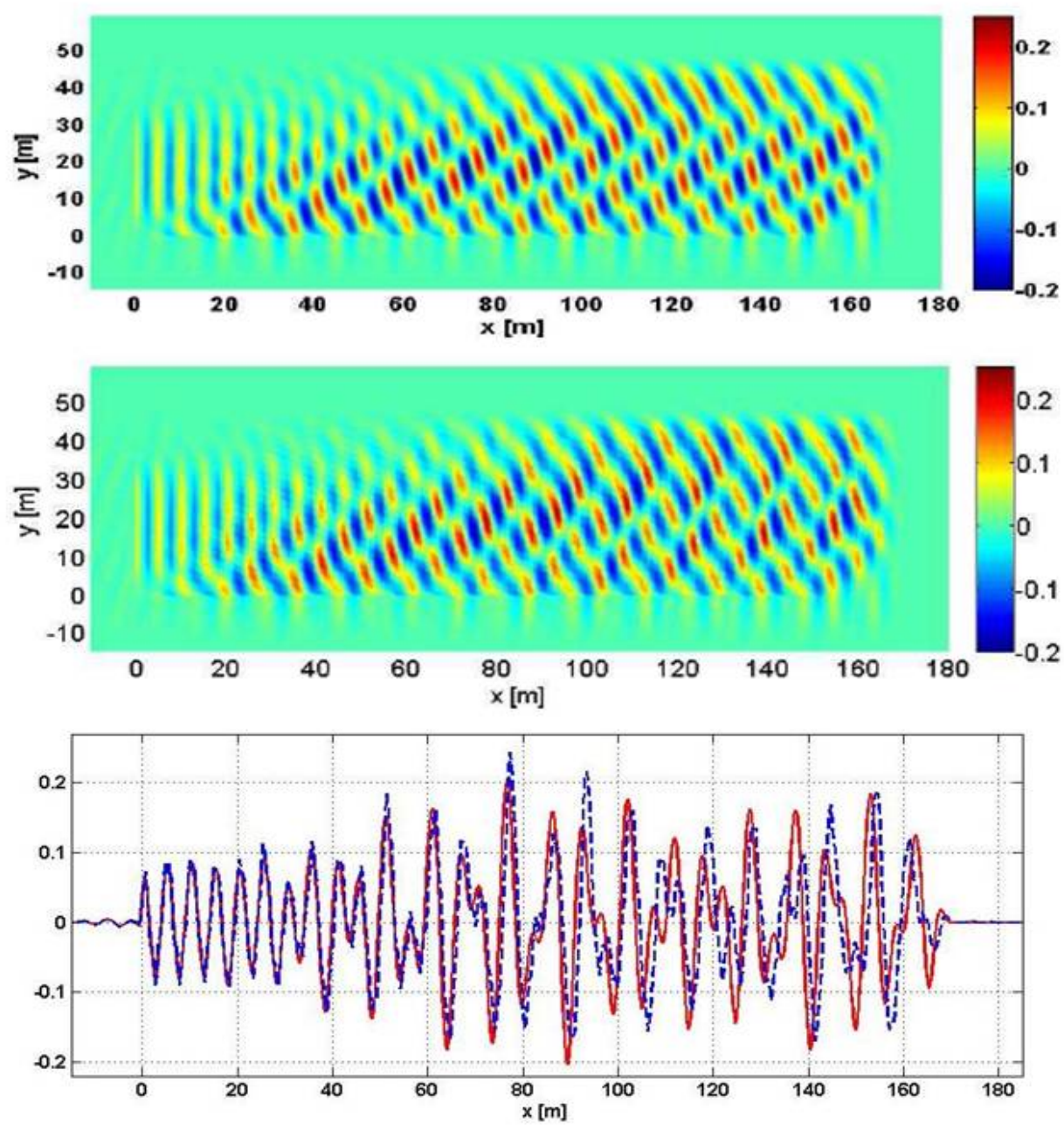

Figure 4.12: Simulation of the linear and the nonlinear AB2-equation for MARIN test no. 122002-122004. The first and the second row are the density plot from the linear and the nonlinear simulation respectively. The last row is the wave profile (in $[m]$ ) after $350[s]$ at $y=19.42[m]$, the solid red line is the linear AB2 and the dashed-blue line is the nonlinear AB2. 
Deviations from the maximal value 1, the correlation defined in this way measures errors in the shift of the simulation; if the correlation is -1 the simulation is in counter phase with the measurement. The variancequotient gives an indication about the amplitude errors between the two signals. The length of the time interval over which the correlation and the variance-quotient have been calculated is from $t=50[\mathrm{~s}]$ until the end of the simulation, i.e $t=350[s]$. In this way we do not count the entrance (or the initialization) effect of the wave generation.

\begin{tabular}{|c|c|c|c|c|}
\hline \multirow{2}{*}{ Position } & \multicolumn{2}{|c|}{ Linear AB2 } & \multicolumn{2}{c|}{ Nonlinear AB2 } \\
\cline { 2 - 5 } & Correlation & $\begin{array}{c}\text { Variance } \\
\text { Quotient }\end{array}$ & Correlation & $\begin{array}{c}\text { Variance } \\
\text { Quotient }\end{array}$ \\
\hline$X=80[\mathrm{~m}]$ & 0.96 & 1.063 & 0.93 & 1.062 \\
\hline$X=90[\mathrm{~m}]$ & 0.95 & 1.01 & 0.9 & 1.03 \\
\hline$X=110.48[\mathrm{~m}]$ & 0.85 & 0.9 & 0.91 & 0.93 \\
\hline$X=130[\mathrm{~m}]$ & 0.9 & 0.96 & 0.94 & 0.9 \\
\hline
\end{tabular}

Table 4.2: Quantitative information about the accuracy of the wave generation using the linear and the nonlinear AB2 for the MARIN test no. 122002-122004.

From table (4.2) we notice that at $x=80[\mathrm{~m}]$ and $x=90[\mathrm{~m}]$ the correlation value of the linear AB2-simulation are higher than the nonlinear AB2-simulation and lower at $x=110.48[\mathrm{~m}]$ and $x=130[\mathrm{~m}]$.

The comparison between the analytic solution and the numerical simulation of the linear AB2-equation is presented in figure (4.13). Here the graphs of the analytic solution is obtained by plotting expression (4.4) with the same parameters as used for the linear AB2-simulation. Shown in figure (4.13) are the density plots of the wave elevation after $t=350[s]$, the time signals at $(80,19.42)$, the time signals at $(130,19.42)$ and the wave profiles at $y=19.42[\mathrm{~m}]$ after $350[\mathrm{~s}]$. Even though the phase of the propagating wave seems to match between the analytic solution and the linear AB2-simulation, from the graph of the density and the wave profile we see 
differences in amplitude. This occurs due to the fact that for the simulation we influx waves from a slit both in the $x$-axis and the $y$-axis while the analytic solution assumes that the waves are infinitely long. Influxing from the slit then introduces diffraction at the end point of the slit. These diffraction waves interfere and propagate with the 'main' propagating waves. It changes the amplitude of the 'main' propagating waves yet apparently it has no effect on the phase.

In order to present the contributions over the long range of frequencies, we show in figure (4.14) the normalized amplitude type of spectra of the measurements and simulations. That is, we plot the absolute value of the (temporal) Fourier transform of the signal and normalized by the highest spectrum value. The spectra show that the linear simulation is only capable to capture the wave evolution in the lowest order which is related to $\omega_{1}=$ 3.49 and $\omega_{2}=2.89$ ). On the other hand, the nonlinear simulation is quite capable to simulate the second order contribution to the spectrum (related to $2 \omega_{1}=6.98,2 \omega_{2}=5.78$ and $\omega_{1}+\omega_{2}=6.38$ ). The other higher order contributions are also present yet they are not accurately simulated since the nonlinear AB2-equation used in this numerical simulation is exact up to (and including) the second order only.

\section{MARIN test case number 128001-128003}

For the simulation of these test cases we influx from the $y$-axis a harmonic wave with amplitude $0.07[\mathrm{~m}]$ and period $1.925[\mathrm{~s}]$ while from the $x$-axis we influx a harmonic wave with amplitude $0.1[\mathrm{~m}]$ and period $2.0493[s]$. We add phase $\phi_{1}=6.904$ for the influxing at the $y$-axis and $\phi_{2}=8.026$ for the influxing at the $x$-axis. These parameters are obtained from the fitting procedure in such a way that the error between the measurement signal at $(x, y)=(80,19.42)$ and linear wave theory is as small as possible. We observe that these amplitudes differ from the prescribed amplitudes as given in table (4.1); $20 \%$ lower for influxing at the short side and $10 \%$ higher for influxing at the the long side. 

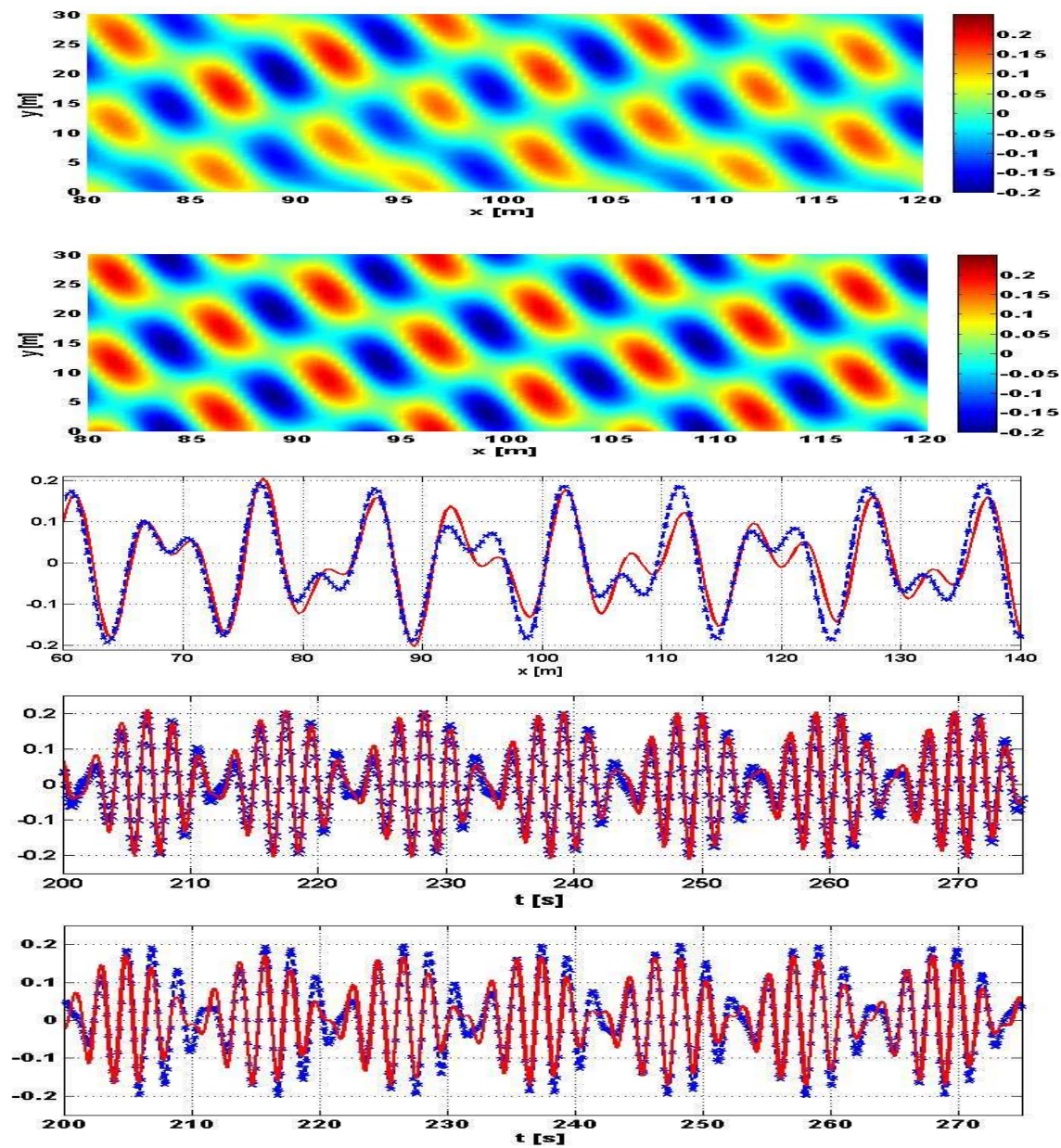

Figure 4.13: Simulation of the linear AB2-equation and the analytic solution for MARIN test no. 122002-122004. The first and the second row are the density plot from the linear simulation and the analytic solution respectively at time $350[\mathrm{~s}]$. The third row is the wave profile at $t=350[\mathrm{~s}], y=19.42[\mathrm{~m}]$. The forth and the fifth row are the time signal at $(80,19.42)$ and at $(130,19.42)$ respectively. From row 3 until row 5, the solid line indicates the linear AB2 and the crossed line indicates the analytic solution. 

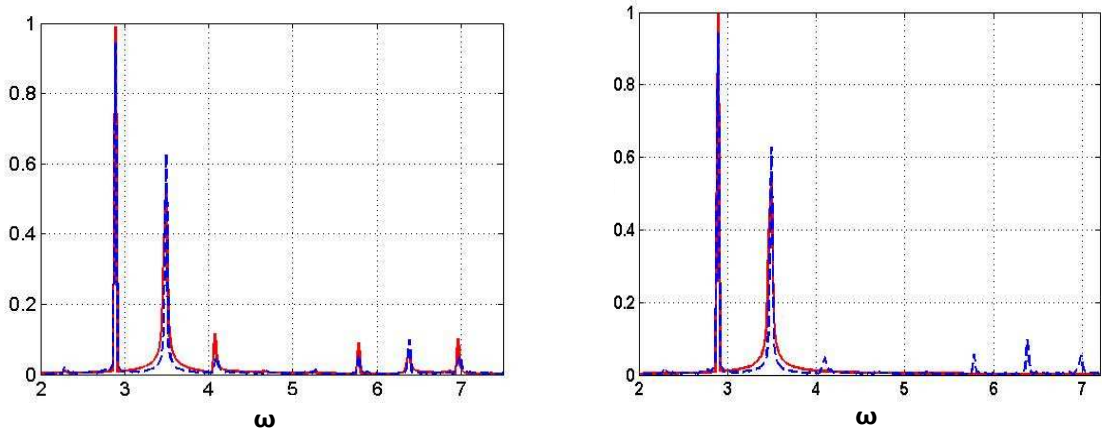

$\boldsymbol{\omega}$
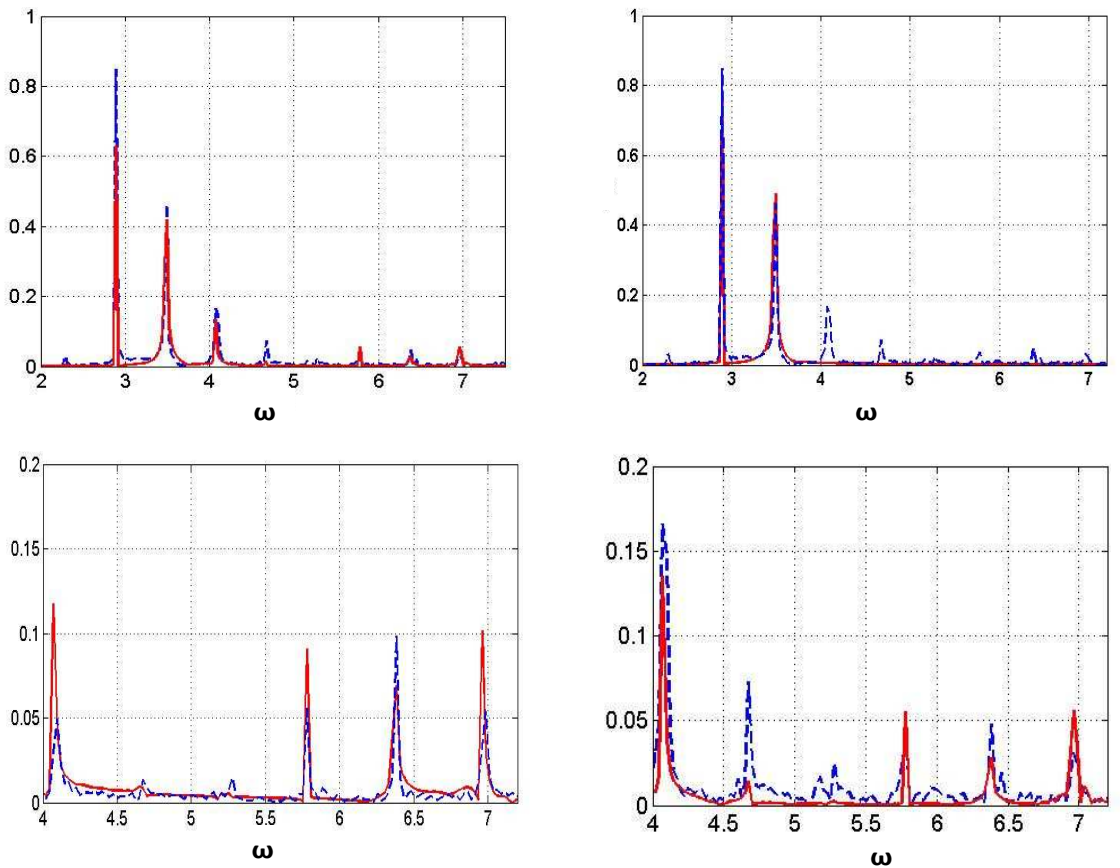

Figure 4.14: Comparison between simulation (solid) and measurement (dashed) for MARIN test no. 122002-122004: the first and second row are plots of amplitude spectra of the nonlinear AB2 (left panel) and the linear AB2 (right panel) against MARIN measurements at position $(x, y)=(80,19.42)$ and $(x, y)=(130,19.42)$ in successive rows. The third row is the zoom out of the nonlinear plot at $x=80$ (left) and at $x=130$ (right) . 
Similar to the previous test cases, here we present the result of simulation using the linear AB2-model (figure (4.15)) and the nonlinear AB2model (figure (4.16)). We show the simulated elevation at each position after $350[s]$ and simulation signals at indicated postions. From these figures we observe that the embedded wave generation model with the linear AB2-equation captures most of the waves deformation and the nonlinearity makes an improvement at the crest and at the trough of the waves. The wave profile of the linear and the nonlinear AB2-equation at $y=19.42[\mathrm{~m}]$ after $350[s]$ are plotted together and shown in figure (4.17).

Table (4.3) shows the correlation and the variance-quotient between the simulation and the measurement signal. We see from this table that for test case number 128001-128003 the difference between the linear and the nonlinear AB2-model is less than $1 \%$ as measured by these quantities; but the graphs and the spectra show that the differences in energy partition and consequently in wave form are quiet substantial.

Figure (4.18) shows the comparison between the analytic solution and the simulation of the linear AB2-equation. Shown in this figure are the density plots of the wave elevation after $t=350[s]$, the time signals at $(80,19.42)$, the time signals at $(130,19.42)$ and the wave profiles at $y=$ $19.42[\mathrm{~m}]$ after $350[\mathrm{~s}]$.

In figure (4.19) we show the normalized amplitude type of spectra of the measurements and simulations. That is, we plot the absolute value of the (temporal) Fourier transform of the signal and normalized by the highest spectrum value. Similar to the test case no. 122002-122004, the linear simulation is only capable to capture the wave evolution in the lowest order which is related to $\omega_{1}=3.264$ and $\omega 2=3.066$. On the other hand, the nonlinear simulation is quite capable to simulate the nonlinear (second order) contribution to the spectrum which is related to $2 \omega_{1}=6.528,2 \omega_{2}=6.132$ and $\omega_{1}+\omega_{2}=6.33$. 

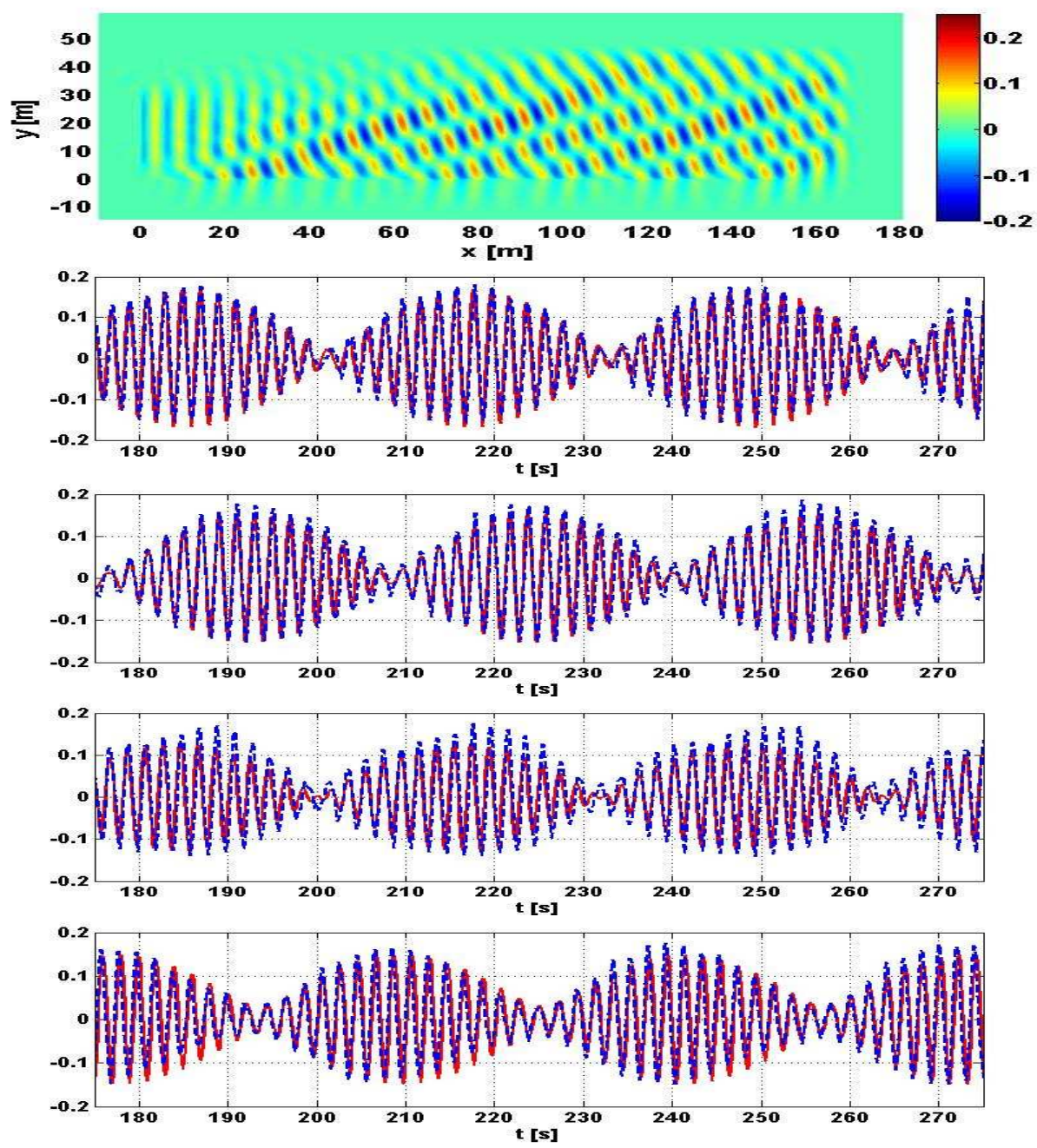

Figure 4.15: MARIN experiments and linear AB2-simulation: MARIN test no. 128001-128003. The first row is density plot after $t=350[s]$. From row 2 until row 5 successively are plots of time traces of the linear AB2-simulation (solid red) and the MARIN measurement (dashed-blue) at $y=19.42[\mathrm{~m}]$ and $x=80,90,110.48,130[m]$. 

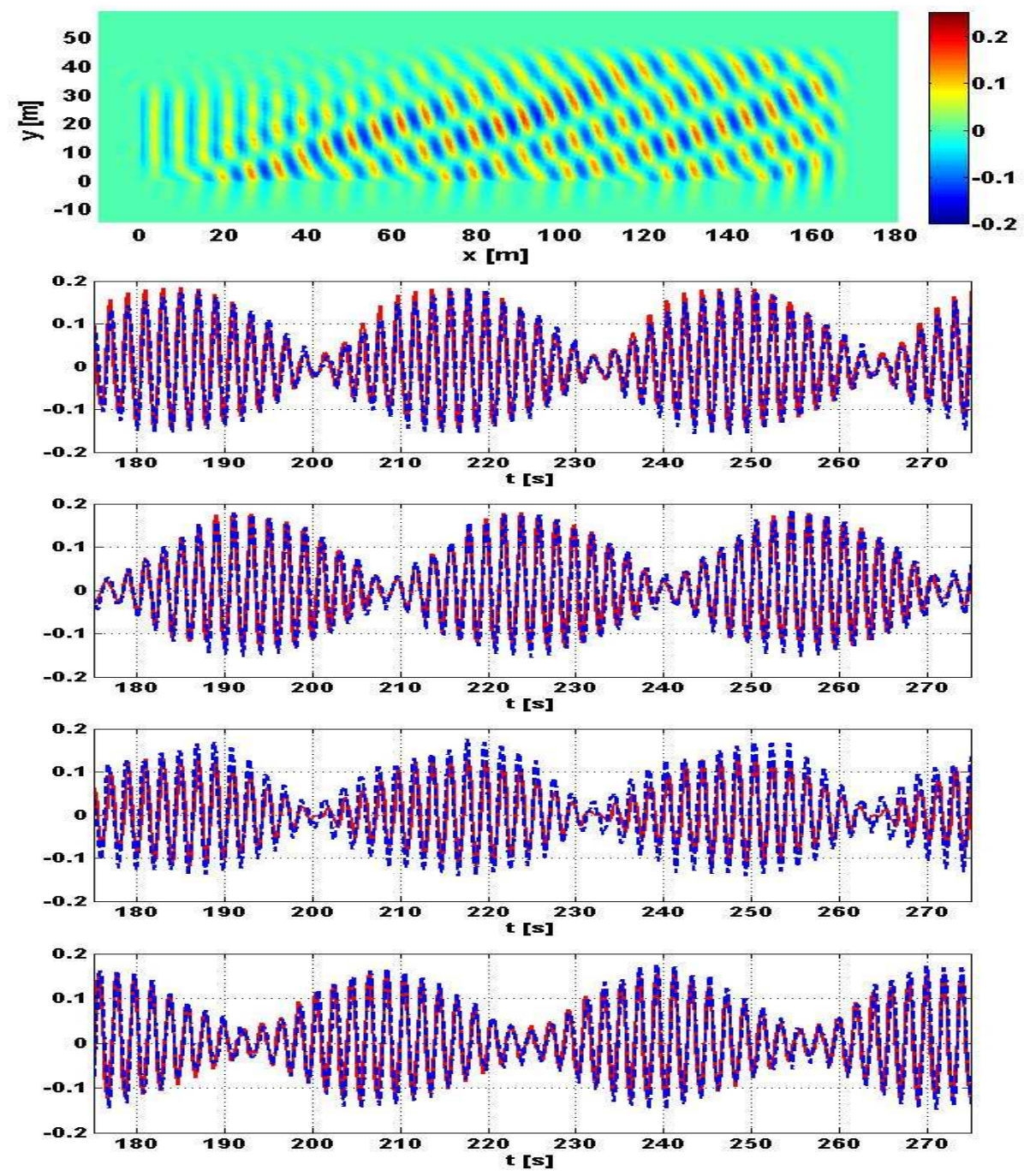

Figure 4.16: MARIN experiments and nonlinear AB2-simulation as in figure (4.15): MARIN test no. 128001-128003. 

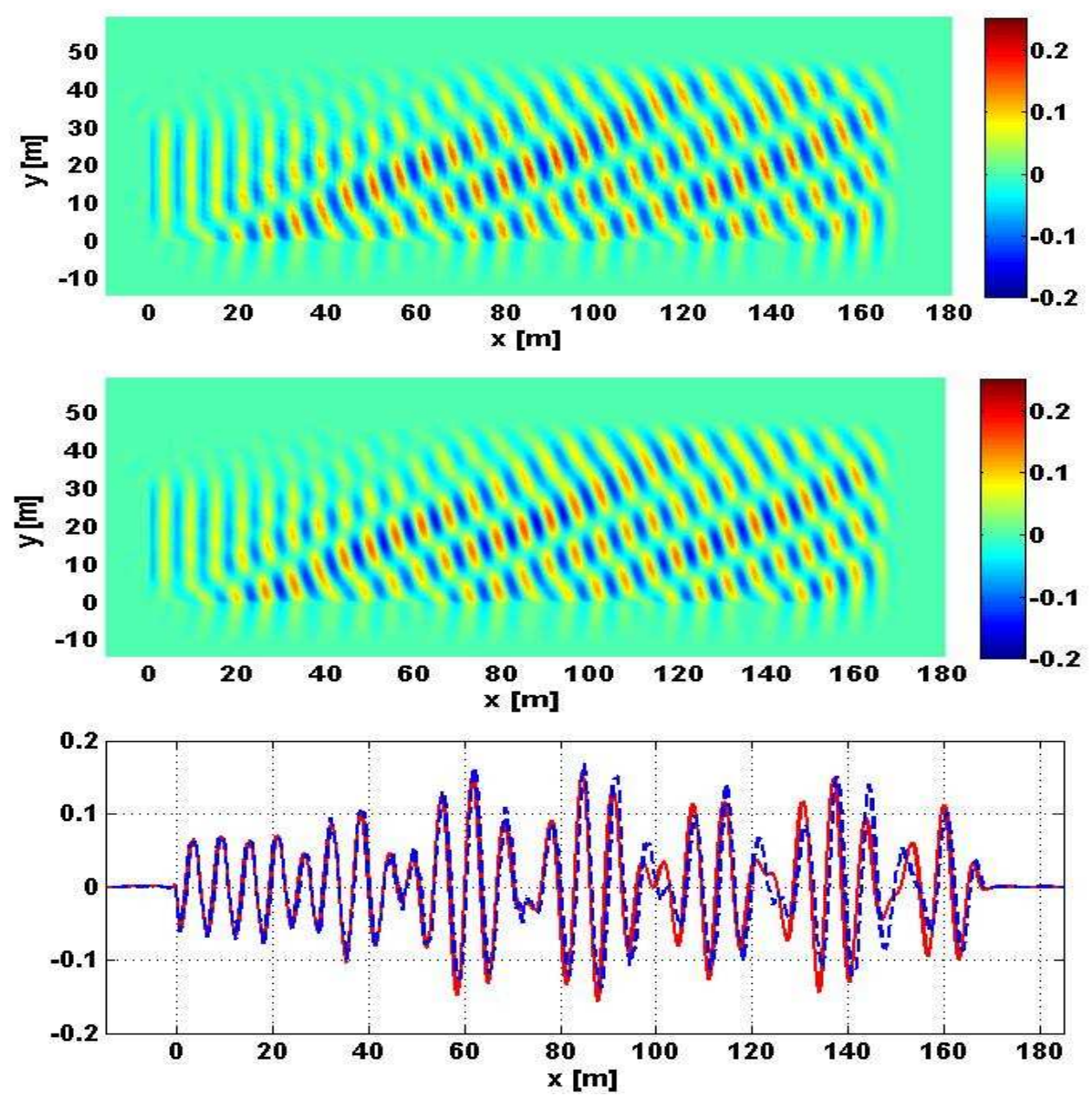

Figure 4.17: Simulation of the linear and the nonlinear AB2-equation for MARIN test no. 128001-128003. The first and the second row are the density plot from the linear and the nonlinear simulation respectively. The last row is the wave profile (in $[m]$ ) after $350[s]$ at $y=19.42[m]$, the solid red line is the linear AB2 and the dashed-blue line is the nonlinear AB2. 

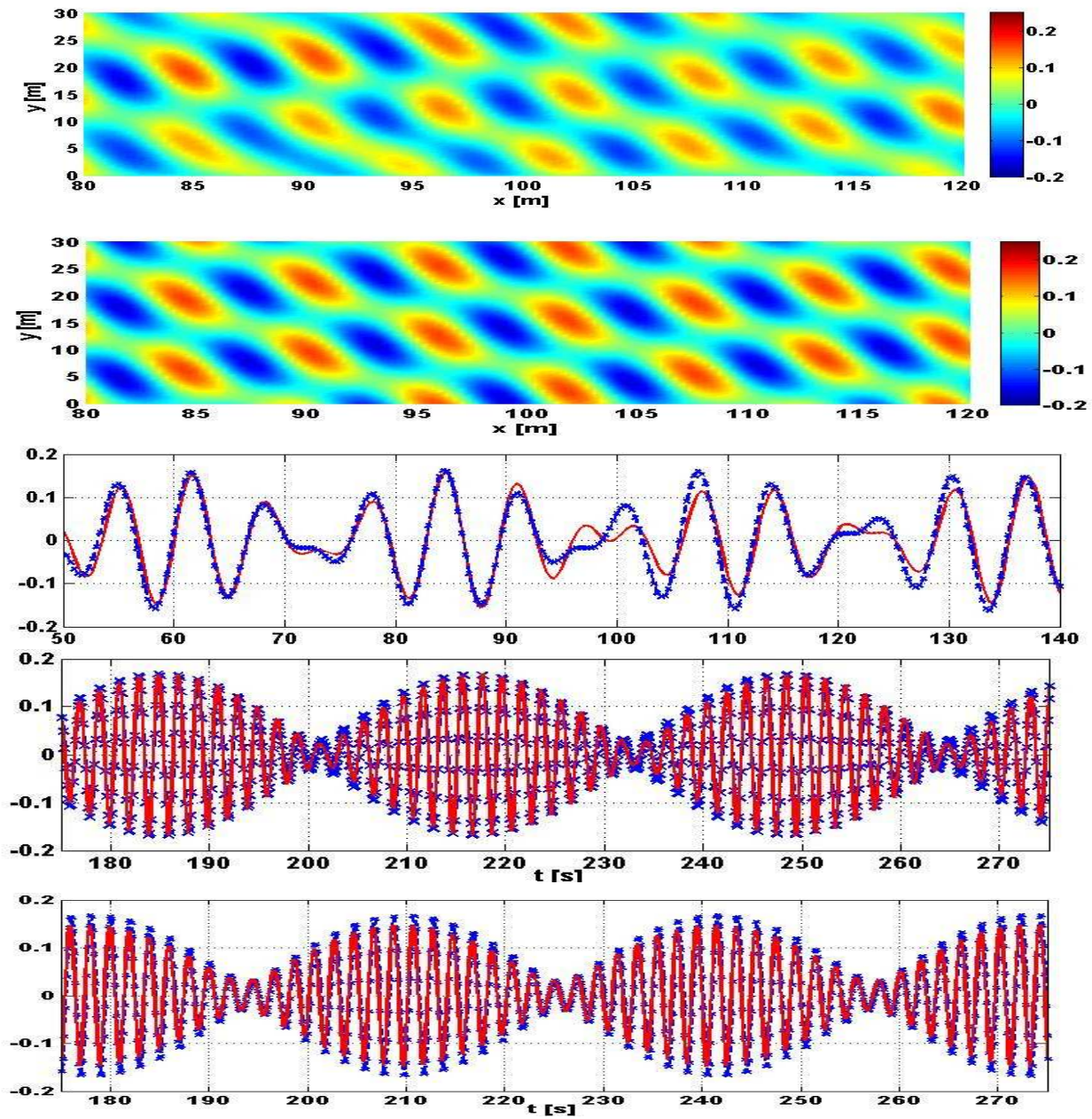

Figure 4.18: Simulation of the linear AB2-equation and the analytic solution for MARIN test no. 128001-128003. The first and the second row are the density plot from the linear simulation and the analytic solution respectively at time $350[\mathrm{~s}]$. The third row is the wave profile at $t=350[\mathrm{~s}], y=19.42[\mathrm{~m}]$. The forth and the fifth row are the time signal at $(80,19.42)$ and at $(130,19.42)$ respectively. From row 3 until row 5 , the solid line indicates the linear $\mathrm{AB} 2$ and the crossed line indicates the analytic solution. 

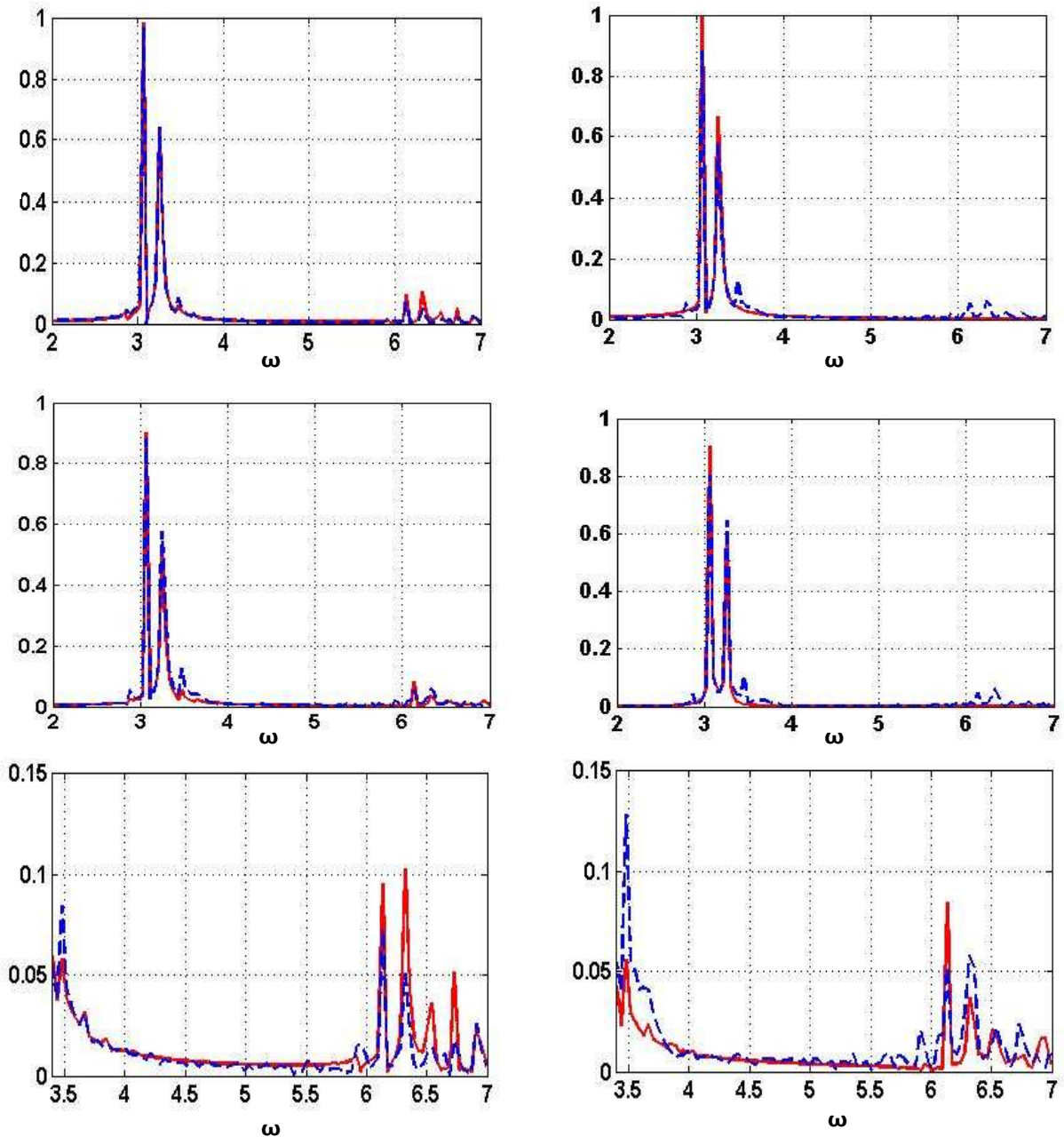

Figure 4.19: Comparison between simulation (solid) and measurement (dashed) for MARIN test no. 128001-128003: the first and second row are plots of amplitude spectra of the nonlinear AB2 (left panel) and the linear AB2 (right panel) against MARIN measurements at position $(x, y)=(80,19.42)$ and $(x, y)=(130,19.42)$ in successive rows. The third row is the zoom out of the nonlinear plot at $x=80$ (left) and at $x=130$ (right) . 


\begin{tabular}{|c|c|c|c|c|}
\hline \multirow{2}{*}{ Position } & \multicolumn{2}{|c|}{ Linear AB2 } & \multicolumn{2}{c|}{ Nonlinear AB2 } \\
\cline { 2 - 5 } & Correlation & $\begin{array}{c}\text { Variance } \\
\text { Quotient }\end{array}$ & Correlation & $\begin{array}{c}\text { Variance } \\
\text { Quotient }\end{array}$ \\
\hline$X=80[\mathrm{~m}]$ & 0.982 & 1.011 & 0.99 & 1.003 \\
\hline$X=90[\mathrm{~m}]$ & 0.979 & 0.923 & 0.986 & 0.96 \\
\hline$X=110.48[\mathrm{~m}]$ & 0.941 & 0.79 & 0.974 & 0.82 \\
\hline$X=130[\mathrm{~m}]$ & 0.98 & 1.01 & 0.986 & 0.98 \\
\hline
\end{tabular}

Table 4.3: Quantitative information about the accuracy of the wave generation using the linear and the nonlinear AB2 for the MARIN test no. 128001-128003.

\subsection{Conclusion}

In this chapter a series of experimental results and numerical simulations on two plane-oblique wave interaction have been presented. For the experiments we influxed plane waves from the short and the long side of the water basin simultaneously. The result of the experiments shows that the colliding plane waves will create (two dimensional) bichromatic waves. The nonlinear behavior of these bichromatic waves are found in the experiments: the waves envelope are deformed and the waves amplitude are higher.

Influxing from the two sides of the basin is very sensitive to the amplitude of the influx signals; for the case of bichromatic waves, if one amplitude is smaller than the other the effect will be noticeable in the zero crossing in between wave envelope.

Using pseudo-spectral method for the implementation of the wave generation model, it is shown that on the length scale of physical basin, the numerical and the experimental results are in satisfactory agreement; the influxing model with the AB2-equation can capture the main aspects of the evolution of the waves. 


\section{Chapter 5}

\section{Conclusions and recommendations}

\subsection{Conclusions}

Many investigations have been made to model the propagation of water waves since the $17^{\text {th }}$ century. The challenges in deriving good wave models come from the frequency dispersion and the nonlinearity. The frequency dispersion is the relation that describes how waves with different wavelength travel at different speed. Nonlinearity is the wave phenomenon that there is energy exchange between different wave modes, showing itself in the effect that waves are sharper at the crests and flatter at the troughs. The classical $\mathrm{KdV}$-equation and the KP-equation have limitations in both aspects, i.e. waves are assumed to be weakly nonlinear and mildly dispersive.

In chapter 2, the AB2-equation that describes two dimensional forward propagating dispersive nonlinear waves has been derived. The derivation of the AB2-equation follows from the variational principle of Miles. The AB2-equation is accurate for waves in arbitrary water depth and accurate up to and including second order. In the lowest order the AB2-equation is an improvement of the KP-type of equation and for waves that have no dependence on the $y$-horizontal direction, i.e. waves travel uniformly in 
the positive $x$-direction, the AB2-equation is exactly the same as the ABequation derived earlier in 2007 by Van Groesen \& Andonowati [46]. The AB2-equation can be implemented easily with a pseudo-spectal method.

In chapter 3, embedded influxing methods for dispersive waves for both one and two horizontal space dimension have been presented. The idea behind embedded influxing is that instead of solving a homogeneous boundary signaling problem, we solve an inhomogeneous problem by adding a source function into the equation of motion. The source term is then determined in such a way that the prescribed signal is obtained as solution. This is a generalization of Duhamel's principle [62]. As shown in chapter 3, the source term depends on the dispersive property of the model; in Fourier space it is proportional to the multiplication between the group speed and the prescribed signal.

Even though the source function is determined uniquely in Fourier space when the dispersion relation is satisfied, yet in physical space the source is not unique. Two limiting cases are area influxing and point (or line) influxing. For the area influxing the influx signal is spread over an area with support the relevant interval of the inverse Fourier transform of the group speed. In deep water this interval will be very long, possibly extending the area of interest of simulations. Therefore to be practical it is recommended to use a multiplicative combination by taking for instance the spatial generation function to be some fractional power $\alpha$ of the group velocity and multiplying the influx signal by the power $(1-\alpha)$ of the group velocity. For the point (or line) influxing the influx signal is convoluted with the group speed function.

Influxing in $\mathrm{KdV}$ type of equations will give waves that propagate in one direction; influxing in Boussinesq type of equations will give waves that propagate in all desired directions (forward and backward). For wave simulation in a laboratory or waves from deep ocean towards the shore, it is advantageous to have one directional influxing method for Boussinesqequation. This can be achieved as shown in chapter 3 by combining a symmetric and an anti-symmetric influxing. The symmetric and the antisymmetric influxing are done by a proper choice of the spatial function in the source term. The method of combining the symmetric and the anti- 
symmetric influxing can be seen as a method to model damping in an embedded way.

A partial verification of the AB2-equation and the two dimensional embedded influxing technique has been presented in chapter 4 . The verification is done by comparing the results from the numerical simulations and the measurement data from the experiments in MARIN hydrodynamics laboratory. The experiments are performed by influxing two harmonic waves with different period from the two sides of the tank. The two dimensional experiments are harder to analyze due to the insecurities in the involved parameters; i.e. angle of propagation, wave periods and wave amplitudes. By using optimal parameters for the numerical simulation, the AB2-equation and the embedded influxing method are accurate over the physical length of the water tank. In this dissertation the optimal parameters are determined in such a way that the least square error between the measurement signal in the middle of the water tank $(x=80[m], y=19.42[m])$ and linear wave theory is as small as possible.

\subsection{Recommendations}

For further research the following outlooks are given:

- The source function for the waves influxing given in this dissertation is derived based on the linear theory. This can be improved by incorporating the second order theory as in [39] .

- In this dissertation only MARIN test case number 122002-122004 and 128001-128003 are simulated and compared. The other test cases still need to be simulated and analyzed; this will be done in a forthcoming paper (M. Hafiizh, Lie S.L \& E. van Groesen, Nonlinear oblique wave collison: comparison experiments and simualtions, to be published.)

- Both the KP and the KdV-equation have a non-periodic profile, namely soliton, as solution. It has not been shown yet whether the ABequation and the the AB2-equation have such soliton solutions. 


\section{Bibliography}

[1] M.J. Ablowitz and P.A. Clarkson. Soliton, nonlinear evolution equations and inverse scattering. Cambridge University Press, 1991.

[2] D. Adytia. Coastal zone simulations with Variational Boussinesq modelling. PhD thesis, University of Twente, The Netherlands, 2010.

[3] D. Adytia and E. van Groesen. Optimized variational 1D Boussinesq modelling of coastal waves propagating over a slope. Coast. Engng 64: 139-150, 2012.

[4] H. Bateman. Notes on a differential equation which occurs in the twodimensional motion of a compressible fluid and the associated variational problems. Proc. R. Soc. Lond. A, 125, 598-618, 1929.

[5] L.J.F Broer. On the hamiltonian theory of surface waves. Appl.Sci.Res. 29: $430-446,1974$.

[6] A. Constantin and J. Escher. Particle trajectories in solitary water waves. Bull. Amer. Math. Soc. 44: 423-431, 2007.

[7] Alex D.D. Craik. The origins of water wave theory. Annu. Rev. Fluid Mech. 36:1-28, 2004.

[8] Alex D.D. Craik. George Gabriel stokes on water wave theory. Annu. Rev. Fluid Mech. 37:23-428, 2005. 
[9] D.H.Peregrine. Long waves on a beach. J. Fluid Mech,27, pp.815-827, 1967.

[10] M. W. Dingemans. Water wave propagation over uneven bottoms. Advanced Series on Ocean Engineering. 13. World Scientific, Singapore, 1997.

[11] D. Dommermuth. The initialization of nonlinear waves using an adjustment scheme. Wave motion, 32 : 307-317, 2000.

[12] B. Engquist and A. Majda. Absorbing boundary conditions for the numerical simulation of waves. Math.Comp 629-651, 1977.

[13] J. Fenton. A fifth-order stokes theory for steady waves. Coastal and Ocean Engineering. Vol. 111 : 216-234, 1985.

[14] Robert G.Dean and Robert A.Dalrymple. Water wave mechanics for engineers and scientists. Advanced Series on Ocean Engineering. 2. World Scientific, Singapore, 1991.

[15] D. Henderson, M. Patterson, and H. Segur. On the laboratory generation of two-dimensional, progressive, surface waves of nearly permanent form on deep water. J. Fluid Mech. 559 : 413-427, 2006.

[16] Leo H. Holthuijsen. Waves in Oceanic and Coastal Waters. Cambridge, 2007.

[17] http://www-history.mcs.st and.ac.uk/Biographies/Laplace.html.

[18] R.S. Johnson. A Modern Introduction to The Mathematical Theory of Water Waves. Cambridge Univ.Press, United Kingdom, 1997.

[19] B.B. Kadomtsev and V.J. Petviashvili. On the stability of solitary waves in weakly dispersive media. Soviet Phys: 539-541, 1970.

[20] G. Kim, C. Lee, and K. Suh. Internal generation of waves: Delta source function method and source term addition method. Ocean Engng 34 : 2251-2264, 2007. 
[21] G. Klopman. Variational Boussinesq modelling of surface gravity waves over bathymetry. $\mathrm{PhD}$ thesis, University of Twente, The Netherlands, 2010.

[22] G. Klopman, E. van Groesen, and M. Dingemans. A variational approach to Boussinesq modelling of fully non-linear water waves. $J$. Fluid Mech. 657 : 36-63, 2010.

[23] I. Lakhturov, D. Adytia, and E. van Groesen. Optimized variational $1 \mathrm{~d}$ Boussinesq modelling for broad-band waves over flat bottom. Wave Motion 49 : 309-322, 2012.

[24] A. L. Latifah and E. van Groesen. Coherence and predictability of extreme events in irregular waves. Nonlin. Processes Geophys., 19, 199-213, 2012.

[25] C. Lee, G. Kim, and K.D. Suh. Extended mild-slope equation for random waves. J. Coast. Eng. 48, pp.277-287, 2003.

[26] C. Lee, W.S. Park, Y. Cho, and K.D. Suh. Hyperbolic mild-slope equations extended to account for rapidly varying topography. J. Coast. Eng. 34, pp.243-257, 1998.

[27] Paul C. Liu. A chronology of freaque wave encounters. Geofizika,24, $1,2007$.

[28] L.Paumond. Towards a rigorous derivation of the fifth order KPequation. Math.Comp.Simulation : 477 - 491, 2005.

[29] J. C. Luke. A variational principle for a fluid with a free surface. J.Fluid.Mech,27: 395-397, 1967.

[30] P.A. Madsen and O.R.Sorensen. A new form of the Boussinesq equations with improved linear dispersion characteristic: part II. A slowlyvarying bathymetry. Coastal Engng: 183-204, 1992.

[31] Masayoshi.T, Toshio.M, and Riichi.I. On the generalized Korteweg de Vries equation. Proc. Japan Acad. 46, 1970. 
[32] S.R. Massel. Extended refraction-diffraction equation for surface waves. J. Coast.Eng.19, pp. 97-126, 1993.

[33] J. W. Miles. On hamilton's principle for surface waves. Journal of Fluid Mechanics 83 (1): 153158, 1977.

[34] O. Nwogu. Alternative form of Boussinesq equations for nearshore wave propagation. Journal of Waterway. Port Coastal and Ocean Engineering, Vol.119, No.6, pp. 618-638, 1993.

[35] Y. Pomeau, A. Ramani, and B. Grammaticos. Structural stability of the Korteweg-de Vries solitons under a singular perturbation. Physica D. 31 (1): 127-134, 1988.

[36] R.C.T. Rainey and Michael S. Longuet-Higgins. A close one-term approximation to the highest Stokes wave on deep water. Ocean Engineering,33: 2012-2024, 2005.

[37] M.M. Rienecker and J.D. Fenton. A Fourier approximation method for steady water waves. J.Fluid.Mech : 119 - 137, 1979.

[38] H.A. Schaffer. Second order wavemaker theory for irregular waves. Ocean Engng 23 : 47-88, 1996.

[39] H.A. Schaffer and CM Steenberg. Second-order wavemaker theory for multidirectional waves. Ocean Engng 30 : 1203-1231, 2003.

[40] R. Shapiro. Smoothing, filtering, and boundary effects. Rev. Geophys. and Space Phys. 8: 359-387, 1970.

[41] Lie S.L, D. Adytia, and E. van Groesen. Embedded wave generation for dispersive surface wave models. Ocean Engineering, Submitted 2013.

[42] Lie S.L and E. van Groesen. Variational derivation of improved KPtype of equations. Phys.Lett.A, 374: 411-415, 2010.

[43] C.T. Stansberg. On the nonlinear behaviour of ocean wave groups. American Society of Civil Engineering 2: 1227-1241, 1997. 
[44] K.D. Suh, C. Lee, and W.S. Park. Time-dependent equations for wave propagation on rapidly varying topography. J. Coast. Eng. 32, pp.91117, 1997.

[45] Gotkas U and Hereman W. Symbolic computation of conserved densities for systems of nonlinear evolution equations. J. Symbolic Computation,1, 1-31, 1999.

[46] E. van Groesen and Andonowati. Variational derivation of KdV-type of models for surface water waves. Phys.Lett.A,366: 195-201, 2007.

[47] E. van Groesen and Andonowati. Fully dispersive dynamic models for surface water waves above varying bottom, part 2: Model equations. Wave Motion 48: 657-666, 2011.

[48] E. van Groesen and I.v.d. Kroon. Fully dispersive dynamic models for surface water waves above varying bottom, part 2: Hybrid spatialspectral implementations. Wave Motion 49: 198-211, 2012.

[49] E. van Groesen and J. Molenaar. Continuum Modeling in the Physical Sciences. SIAM, Philadelphia, 2007.

[50] E. van Groesen, Lie S.L., I. Lakhturov, and Andonowati. Accurate modelling of uni-directional surface waves. J. Comput. Appl. Math: $1747-1756,2010$.

[51] P. J. van Leeuwen and G. Klopman. A new method for the generation of second-order random waves. Ocean Engng. 23(2) : 167-192, 1996.

[52] MARIN's website: www.marin.nl.

[53] G. Wei and J. T. Kirby. Simulation of water waves by boussinesq models. Research report No. CACR-98-02, 1998.

[54] G. Wei and J.T. Kirby. Time-dependent numerical code for extended Boussinesq equations. J.Waterway : 251-261, 1995. 
[55] G. Wei, J.T. Kirby, and A. Sinha. Generation of waves in Boussinesq models using a source function method. Coastal Engng 36: 271-299, 1999 .

[56] J. Westhuis. The numerical simulation of nonlinear waves in a hydrodynamics model test basin. $\mathrm{PhD}$ thesis, University of Twente, The Netherlands, 2001.

[57] J. Westhuis and R.H.M. Huijsmans. MARIN Report No.15309. MARIN, The Netherlands, 2000.

[58] J. Westhuis, E. van Groesen, and R.H.M. Huijsmans. Experiments and numerics of bichromatic wave groups. CoastEOcean Eng 127: 334-342, 2001.

[59] G.B. Whitham. Linear and Nonlinear Waves. John Wiley \& Sons, 1974.

[60] www.wikipedia.org/wiki/Wavefarm.

[61] V. E. Zakharov. Stability of periodic waves of finite amplitude on the surface of a deep fluid. J.Appl.Mech.Tech.Phys 9(2): 190-194, 1968.

[62] Erich Zauderer. Partial Differential Equations of Applied Mathematics. John Wiley \& Sons, Canada, 1989. 